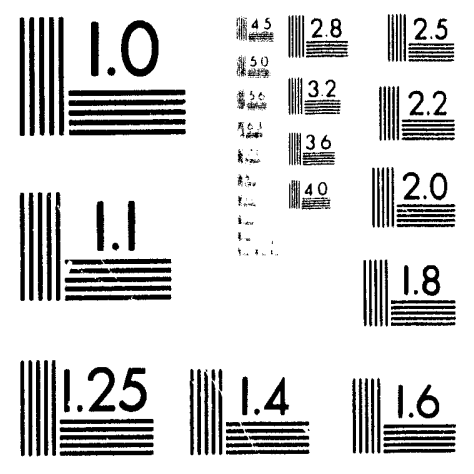



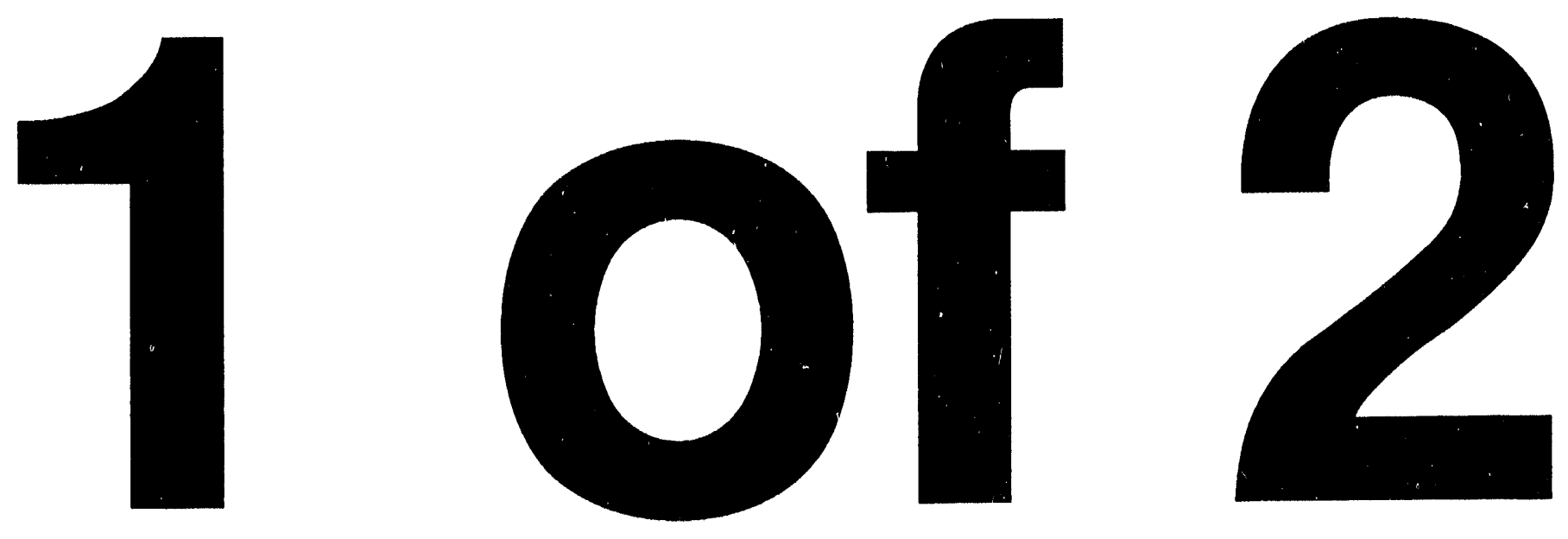


\section{Tank Farm Surveillance and Waste Status Summary Report for May 1993}

B. M. Hanlon

Date Published

August 1993

Prepared for the U.S. Department of Energy Office of Environmental Restoration and

Waste Management

\footnotetext{
(2. Westinghouse P.O. Box 1970

W Hanford Company Richiand, Washington 99352

Hantord Operations and Engineering Contractor for the

U S. Deparment of Energy under Contract DE ACO6-87RL 10930
} 


\section{APPROVAL}
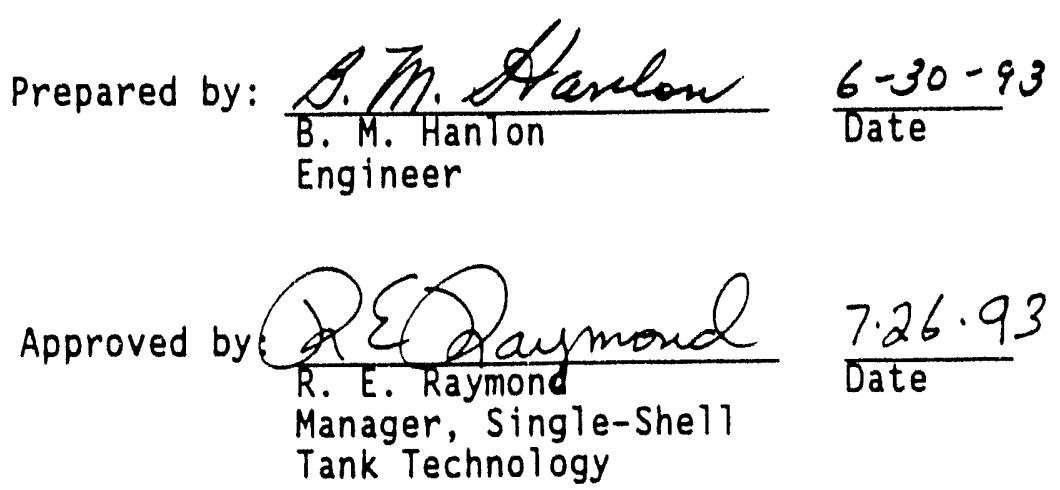


\begin{tabular}{|c|c|c|c|}
\hline Dats/geceiyed: & INFORMATION RELEASE REQIJEST & $-1+12+10$ & $\left\{\begin{array}{l}\text { Weference: } \\
\text { WHC-CM-3-4 }\end{array}\right.$ \\
\hline
\end{tabular}

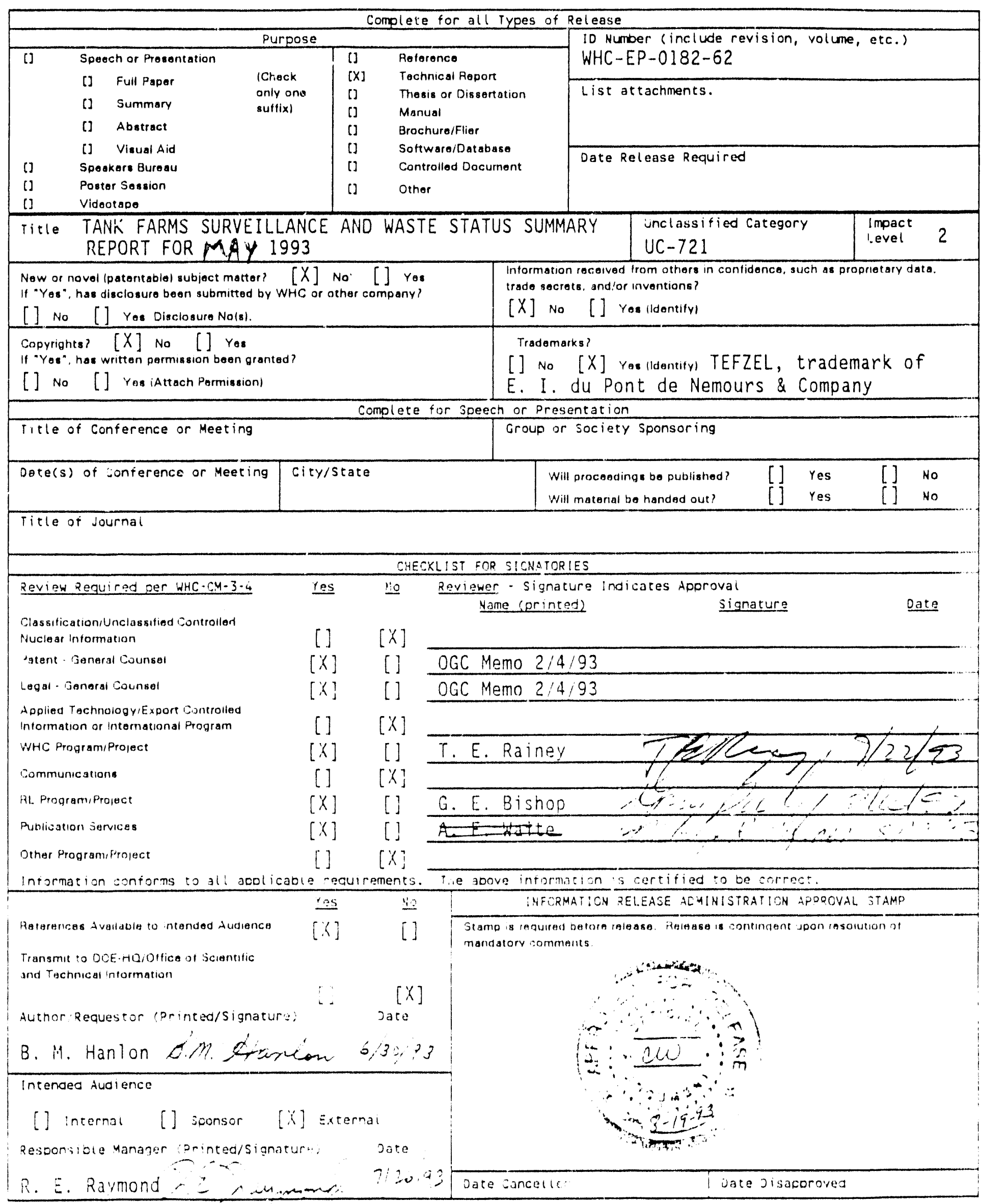




\title{
WHC-EP-0182-62
}

\section{TANK FARM SURVEILLANCE AND WASTE STATUS SUMMARY REPORT FOR MAY 1993}

\author{
B. M. Hanion
}

\begin{abstract}
This report is the official inventory for radioactive waste stored in underground tanks in the 200 Areas at the Hanford Site. Data that depict the status of stored radioactive waste and tank vessel integrity are contained within the report. This report provides data on each of the existing 177 large underground waste storage tanks and 49 smaller catch tanks and special surveillance facilities, and supplemental information regarding tank surveillance anomalies and ongoing investigations. This report is intended to meet the requirement of U.S. Department of Energy-Richland Operations Office Order 5820.2A, Chapter I, Section 3.e. (3) (DOE-RL, 1990, Radioactive Waste Management, U. S. Department of Energy-Richland Operation Office, Richland, Washington) requiring the reporting of waste inventories and space utilization for Hanford Tank Farm Tanks.
\end{abstract}


WHC-EP-0182-62

This page intentionally left blank. 


\section{CONTENTS}

SUMMARY

TANK STATUS

TANK INVESTIGATIONS

HIGHLIGHTS

\section{Appendixes:}

A. TANK AND EQUIPMENT CODE AND STATUS DEFINITIONS ............ A-1

Tank and Equipment Code/Status Definitions . . . . . . . . . . . . A-3

B. TANK FARM CONFIGURATION, STATUS AND FACILITY CHARTS . . . . . . . . . . . B-1

1 High-Level Waste Tank Configuration . . . . . . . . . . . . . . . B-3

2 Double-Shel1 Tank Instrumentation Configuration . . . . . . . . . B-4

3 Single-Shell Tank Instrumentation Configuration . . . . . . . . . B-5

4 Double-Shell Tank Status . . . . . . . . . . . . . . . . . B-6

5200 E Single-Shell Tank Status... . . . . . . . . . . . . B-7

$6200 \mathrm{~W}$ Single-Shell Tank Status... . . . . . . . . . . . . . . B-9

7 Tank Farm Facilities Quick Reference. . . . . . . . . . . . . . B-11

8 Hanford Tank Farms Facilities Chart . . . . . . . . . . . . . . . B-12

C. MONTHLY SUMMARY ......................... . c-1

1 Monthly Summary . . . . . . . . . . . . . . . . . . . . . . C-3

2 Tank Use Summary. . . . . . . . . . . . . . . . . . . . . C-4

3 Inventory Summary by Tank Farm . . . . . . . . . . . . . . . . . C -5

4 Inventory and Status by Tank - Double-Shell Tanks . . . . . . . . C-6

5 Inventory and Status by Tank - Single-Shell Tanks . . . . . . . . C-9

D. PERfORMANCE SUMMARY .................... . . . . . . . . .

1 Perforinance Summary . . . . . . . . . . . . . . . . . . D-3

E. LIQUID StATUS AND PUMPABLE LIQUID REMAINING IN TANKS ........ . E-1

1 Liquid Status and Pumpable Liquid Remaining in Tanks . . . . . . . E-3

F. PUMPING RECORD ................... . F-1

1 Pumping Record . . . . . . . . . . . . . . . . . . . . . . F-3

G. CATCH TANKS AND SPECIAL SURVEILLANCE FACILITIES . . . . . . . . . G-1

1 East and West Area Catch Tanks and Special Surveillance Facilities (Active) . . . . . . . . . . . G-3

2 East Area Catch Tanks and Special Surveillance Facilities (Inactive) . . . . . . . . . . . . . . G-4

3 West Area Catch Tanks and Special Surveillance . . . . . . . . G-5

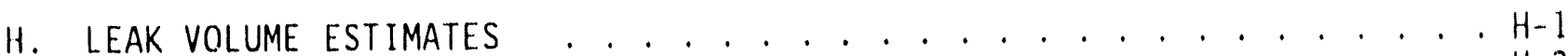

1 Single-Shell Tank Leak Volume Estimates . . . . . . . . . . H-3 
WHC-EP-0182-62

This page intentionally left blank. 


\section{LIST OF TABLES}

1 Watch List Tanks ................... . . 12

2 Tanks Containing $>1,000$ gram mole of Ferrocyanide (Watch l.ist Tanks). . . . . . . . . . . . . . . . 13

3 Tanks with Potential for Hydrogen or Flammable Gas Accumulation Above the Flammability Limit (Watch List Tanks) . . . . . . . . . . 15

4 Tanks Containing Concentrations of Organic Salts $>3 \%$ Weight TOC (Watch List Tanks) . . . . . . . . . . . . . . . 16

5 Single-Shell Tanks with High Heat Loads $(>40,000 \mathrm{Btu} / \mathrm{hr}) . . . . . \quad 17$

6 Double-Shell Tank Waste Type and Space Allocation . . . . . . . . . 19

7 Automatic Food Instrument Company (FIC) Gauges Out of Service. . . 20

8 Single-Shell Tanks Monitoring Compliance Status . . . . . . . . . . 21

9 Double-Shell Tanks Monitoring Compliance Status . . . . . . . . . . 26

\begin{tabular}{|c|c|c|}
\hline \multicolumn{3}{|c|}{ METRIC CONVERSION CHART } \\
\hline 1 inch & $=$ & 2.54 centimeters \\
\hline 1 foot & $=$ & 30.48 centimeters \\
\hline 1 gallon & $=$ & 3.80 liters \\
\hline 1 ton & $=$ & 0.90 metric tons \\
\hline \multicolumn{3}{|c|}{${ }^{\circ} \mathrm{F}=\left(\frac{9}{5}{ }^{\circ} \mathrm{C}\right)+32$} \\
\hline \multicolumn{3}{|c|}{$\begin{array}{c}1 \mathrm{Btu} / \mathrm{h}=2.930711 \mathrm{E}-01 \text { watts } \\
\text { (International Table) }\end{array}$} \\
\hline
\end{tabular}




\section{WHC-EP-0182-62}

This page intentionally left biank 
WHC-EP-0182-62

\section{TANK FARM SURVE LLANCE AND WASTE STATUS SUMMARY \\ KEPORT FOR MAY 1993 \\ SUMMARY}

Note: Changes from the previous month are in bold print.

\section{TANK STATUS}

\begin{tabular}{|c|c|c|}
\hline Category & Quantity & Date of Last Change \\
\hline In-Service Tanksc & 28 double-she 11 & $10 / 86$ \\
\hline Out-of-Service Tanks & 149 single-she 11 & $07 / 88$ \\
\hline Assumed Leaker Tanks ${ }^{f}$ & 68 single-shell & $5 / 93$ \\
\hline Sound Tanks & $\begin{array}{l}28 \text { dcuble-shel1 } \\
82 \text { single-shell } \\
\end{array}$ & $\begin{array}{c}1986 \\
10 / 92 \\
\end{array}$ \\
\hline Interim Stabilized Tanks & 106 single-she 11 & $04 / 93$ \\
\hline Not Interim Stabilized ${ }^{\prime}$ & 43 single-she 11 & $04 / 93$ \\
\hline Interim Isolated Tankse & 98 single-she 11 & $09 / 91$ \\
\hline Watch List Tanks & $\begin{array}{r}48 \text { single-she } 11 \\
6 \text { double-she } 11 \\
\end{array}$ & $\begin{array}{c}1991 \\
11 / 92 \\
\end{array}$ \\
\hline
\end{tabular}

although all 149 single-shell tanks were removed from service (i.e., no longer authorized to receive waste) as of November 21, 1980, the category of "Out-of-Service" was not established until July 1988.

b of the 105 tanks classified as interim stabilized, 58 are listed as assumed leakers. The total of 105 interim stabilized tanks includes eight tanks that do not meet current established supernatant and interstitial liquid stabilization criteria: 104-B, 107-B, 110-A, 111-B, 110-BX, 102-T, 112-T, and 110-U. (These tanks did meet the criteria in existence when they were declared interim stabilized.)

c six double-shell tanks listed as "in service" are currently included on the Hydrogen Watch List and are thus prohibited from receiving waste in accordance with "Safety Measures for Waste Tanks at Hantord Nuclear Reservation," Section 3137 of the National Defense Authorization Act for Fiscal Year 1991, November 5, 1990, Public Law 101-510.

$d$ of the 48 single-shell tanks on Watch Lists, 22 have been Interim Stabilized.

Of the 48 single-shell tanks on Watch Lists, 20 have been Interim Isolated.

Wine of the tanks are both assumed leakers and not Interim Stabilized. See Appendix $H$ for more details.

\section{TANK INVESTIGATIONS}

This section includes all single-shell tanks or catch tanks on the Alert List for surface level or interstitial liquid level (ILL) decreases, or for druwell/lateral radiation level increases.

There were no tanks under investigation for surface or ILL decreases or for drywell/lateral radiation increases during May 1993. 
A. Assumed Leakers or Assumed Re-leakers: (See Appendix A for definition of "Re-leaker")

This section includes all single- or double-shell tanks or catch tanks for which an off-normal or unusual occurrence report has been issued for assumed leaks or re-leaks. Tanks/catch tanks will remain on this list until either a) completion of Interim Stabilization, or b) the updated occurrence report indicates that the tank/catch tank is not an assumed leaker.

Tank 241-BX-111. The surface level showed a slow erratic increasing trend since 1986. The level had increased approximately 1.00 inch from the reference baseline of 79.00 inches. A decreasing trend was observed Juring February 1993, but has since remained stable, fluctuating between 7875 and 79.00 inches during March and April 1993. The decrease criteria for this tank is 1.00 inch from the reference baseline. The liquid observation well (LOW) interstitial liquid level (ILL) was stable as of April 1, 1993.

Resolution status: When this tank was under investigation, preliminary notifications were made to regulatory agencies on March 24 and Apri1 16, 1993. Preparations to begin emergency pumping were initiated in late March. This tank was declared an assumed re-leaker on April 30, 1993. Upon completion of pumping, the tank will he rebaselined.

Tank 241-SX-102. The quarterly Liquid Observation Well (LOW) reading taken April 30, 1993, indicated a decrease of 0.2 feet from the established baseline. The rerun requested and taken on May 5, 1993, indicated a decrease of 0.3 feet which equals the established decrease criteria. Previous neutron LOW readings had been stable, fluctuating between the baseline and a 0.2 -foot decrease, but the May 5, 1993, reading was the first indication of a 0.3 foot or larger decrease. An Off-Normal Report was issued at that time and the LOW frequency was increased from quarterly to weekly. The neutron LOW is the primary means of liquid level detection as this tank has a solid surface. The LOW readings taken on May 20, 1993, indicated a further decrease to 0.4 feet below the established baseline. This exceeds the decrease criteria. The previous off-Normal was upgraded to an Unusual Occurrence Report and proper notifications were initiated. Because of the recently observed decreasing trend, this tank was declared an "assumed leaker" on May 20, 1993.

Resolution status: Emergency pumping efforts for this tank have been initiated, but are complicated by several factors. The tank is on the hydrogen Watchlist and will require extensive safety assessment to determine the safety aspects of pumping liquids out of the tank. An initial characterization period may be required to gather data regarding hydrogen gas generation. In addition, Tank 102-SX contains Complexed Concentrate waste and 1: 1 require detalled sampling and compatibility evaluation to ensure that the

ste is compatible with the chosen receiver tank. There are strong

dications that the waste would have to be sent through the cross-site transfer route, because there $m=y$ not be an acceptable receiver tank in the 200 West Area. This tank is a not yet stabilized, Partial Isolated tank, actively ventilated, with $183 \mathrm{Kgall}$ on drainable liquid. See Highlights Item \#5, Occurrence Report RL-WHC-TFARM-1993-0044, for further information.

\section{B. Tanks with increases indicating possible intrusions:}

This section includes all single-shell tanks for which the surveillance data shows that the surface level or ILL has met or exceeded the increase criteria (this section does not include all tanks on the Alert List because the criteria for the Alert List is $\underline{50 \%}$ of the increase criteria). 
Tank 241-B-202. A steady increase in the surface level measurement has been observed since December 1984. The manual tape pencil plummet is contacting 1iquid. When the quarterly reading was obtained on October 6, 1992, the level was recorded as 144.75 inches, thus exceeding the 2.00 -inch increase criteria from the established baseline of 142.50 inches. The surface level measurement was rechecked on October 9, 1992, (145.50 inches) and October 13 (145.00 inches), verifying the increase and that the criteria had been exceeded. The surface level continues to show an increase and is at 145.50 inches for May 1993. The monitoring frequency has been increased from quarterly to monthiy. Occurrence Report RL-WHC-TANKFARM-1993-0024 was issued February 13, 1993. The tank was administratively stabilized in May 1985.

Resolution status: A photo package was initiated on May 11, 1993, to investigate the possibility of an intrusion. Review of previous photos was inconclusive. New photos are required to determine the actual supernatant increase, if any. A temporary baseline will be established at 145.25 inches, until the new photos are available.

Tank 241-BX-103. On January 18, 1993, the surface level measurement in 103-BX exceeded the 0.50 -inch increase criteria from the reference baseline of 19.50 inches, and was verified on January 20, 1993. The surface level measurement is currently at 20.40 inches. This tank has shown an erratic increase in surface level measurements since January 6, 1986. Discrepancy Report S\&DA 93522 was issued January 21, 1993. Occurrence Report RL-WHC-TANKFARM-1993-0036 was issued March 25, 1993. The FIC plummet is contacting liquid as indicated by in -tank photographs taken October 31, 1986. Tank 241-BX-103 is Sound, and Interim Stabilized/Interim Isolated.

Resolution status: The current level is greater than that prior to stabilization in November 1983. The tank was previously determined to have experienced an intrusion from 1977 to March 1983 (prior to stabilization). Subsequent isolation was expected to halt the intrusion, however, the intrusion is apparently ongoing. A work package was initiated on May 11 , 1993, to re-seal pits and risers and to obtain in-tank photos. The photos will be used to assess the current stabilization status of the tank. A visual survey of the area was performed to determine possible paths for precipitation to enter the tank. The weather covering on the pits and risers was found in place and undamaged. The existing grade is level and revealed no obvious draining problems. Design/isolation drawings will be reviewed to determine possible paths for intrusion.

Tank 241-TX-113. The LOW ILL has shown a slow increase since March 12, 1986, with both the neutron and gamma probes. This tank appeared on the Alert list in 1989, when the ILL reached $50 \%$ of the 0.4 -foot increase criteria, but was removed in 1990 when the ILL appeared stable for longer than a 12-month period. The scan data on February 5, 1993, showed the ILL to be 5.5 foot and at the 0.4-foot increase criteria from the reference baseline. S\&DA

Discrepancy Report 93-534 was issued March 10, 1993. The LOW was 1ast scanned May 14, 1993, and showed the ILL to be 0.3 foot above the baseline. Resolution status: The technical evaluation of this tank was completed April 14, 1993. The results were inconclusive, with recommendation to accelerate the 0ctober 1997 waste characterization of the tank. This characterization is expected to confirm that solids are dissolving, causing an increase in ILL. It is also recommended that new photos be taken of the tank interior. A photo package was initiated on May 15, 1993. A letter will be written in July to request acceleration of waste characterization. LOW scans and surface level readings have been increased from a quarterly to a monthly frequency. 
Tank 241-TX-115. The LOW ILL is exceeding the 0.4 -foot increase criteria from the reference baseline. This tank has been on report since 1988, when the increase criteria was first exceeded. Comparison of past and present in-tank photographs show no significant change in surface conditions or obvious evidence of intrusion. Surveillance Deviation Report \#88-08 was issued on May 18, 1988, and Event Fact Sheet \#TF-EFS-88-140 was issued October 27, 1988. This tank appeared on the Executive Summary Report and Alert List until December 31, 1991, when the report was discontinued. The ILL continues to show an increase with both the neutron and gamma probes. The LOW was last scanned May 14, 1993, and showed an additional 0.2-foot increase from the previous February 2, 1993, LOW reading.

Resolution status: The technical evaluation of the alert condition in this tank was completed April 14, 1993. The results were inconclusive, with recommendation to accelerate the October 1997 waste characterization. Waste characterization is expected to confirm that solids are dissolving, causing an increase in ILL. The 1981 photos show evidence of rain intrusion through a central pump pit riser. However, it cannot be concluded from the 1988 photos that the intrusion is ongoing. A photo package was initiated on May 11, 1993. This tank will be included in the waste characterization with 113-TX. LOW scans and surface level readings have been increased from a quarterly to a monthly frequency.

Tank 241-TY-102. On November 8, 1992, the automatic FIC surface level measurement of 32.10 inches exceeded the 0.50 -inch increase criteria from the reference baseline of 31.40 inches. Discrepancy Report S\&DA-92-489 was issued November 9, 1992. The tank has a history of icicle buildup on the FIC plummet and intrusions. The surface level measurement was 31.80 inches as of May 31 , 1993. Resolution status: This tank is experiencing an ongoing intrusion as is evident by comparison of November 1984 photos with July 1987 photos and an increasing trend in surface level data. A visual survey was conducted to determine possible paths for precipitation to enter the tank. The grade around the tank is level and weather covering is on the pits and intact. The photo package, initiated on May 17, 1993, will be required to assess the current stabilization status of the tank, and to assist in determining the possible paths of intrusion. Design/isolation drawings will be reviewed in July to further determine possible paths of intrusion.

Vent Station Catch Tank. The zip cord surface level reading exceeds the maximum operating limit of 36.00 inches. The manual tape has been out of service since July 7, 1992. A temporary zip cord was installed December 16 , 1992. The level was 45.50 inches which exceeded the active tank 1 imit of $50 \%$ volume of 40 inches ( 400 gallons). Transfers are not permitted until the tank is pumped and the level is within limits. Discrepancy Report S\&DA-92-511 was issued December 24, 1992. A new calibrated zip cord was installed May 26, 1993.

Resolution status: Work packages are out to repair the manual tape and pump the catch tank. Extensive surface contamination in the surrounding area is hampering efforts to perform the needed activities. Sampling of the solution in the tank is necessary before pumping can hegin. Completion of the transfer procedure is expected June 30,1993 . Estimated date of completion of pumping is July 15, 1993.

UX-302-A Catch Tank. Surface level measurement exceeds the maximum operating limit of 50.00 inches. The level is 62.60 inches which exceeds the $50 \%$ volume of 54 inches ( 8840 gallons). The FIC plummet is contacting liquid.

Discrepancy report S\&DA-92-465 was issued May 12̌; 19912. A work package was 
initiated to pressure test lines and to pump liquid level to below alarm limits.

Reselution status: Work packages for the transfer of waste from UX-302-A and the repair of necessary instrumentation are being prepared, and the transfer of waste is being scheduled as a prestart item for the Cross Site Transfer. Completion of transfer procedure is expected June 30, 1993. A work package has been prepared to sample/pump this catch tank. Expected completion date is July 15, 1993.

c. The following tanks have been reported as assumed leakers, and al though shown as Interim Stabilized, they do not meet current Interim stabilization criteria. Surveillance data do not show an indication of a continuing leak.

$\begin{array}{ll}104-B & 110-B X \\ 107-B & 102-T \\ 110-B & 112-T \\ 111-B & 110-U\end{array}$

II. HIGHLIGHTS

1. Criticality Safety Issues

On Apri1 30, 1992, an Unreviewed Safety Question (USQ) concerning criticality safety issues in the Tank Farms was declared to be a reportable event. Unusual 0ccurrence report RL-WHC-TANKFARM-1992-0037 was issued. A prohibition was placed on all waste transfers into and between the tank farm facilities which is negatively affecting various Hanford programs. On September 1, 1992, the approved Justification For Continued Operations (JCO) was received. This establishes the limitations for all tanks receiving transfers and also ex-ludes any interim stabilization of single-shell tanks until further evaluations are completed and approved by DOE-HQ. On December 15, 1992, the approved JCO was issued as WHC-SD-WM-JCO-001, "Justification for Continued Operations of Hanford High Level Waste Tanks Resulting From the Criticality USQ, 492-CRITSAS." Resolution of the USQ is scheduled for summer 1993.

2. Interim Stabilization of Tank 241-T-101

Tank 101-T was declared an assumed leaker on October 4, 1992. Total leakage is assumed to be approximately 7500 gallons. Approval from DOE-HQ to pump the tank was received on February 25, 1993. Pumping was completed on April 6, 1993, total gallons pumped was 25,300. The evaluation for meeting interim stabilization criteria was completed on April 14, 1993, and official notification to regulating agencies declared the tank interim stabilized on May 28, 1993. (Further information appeared in the April report)

\section{Tank 241-C-105}

Evaluation has been completed on the heat load from Tank 24l-C-105. The thermal analysis (WHC-SD-WM-ER-189, Thermal Analysis of Tank 241-C-105 in Support of Process Test, January 1993), indicated the best-estimate heat generation rate for $105-\mathrm{C}$ is approximatley $20,000 \mathrm{Btu} / \mathrm{hr}$, with a conservative upper bound of about 25,000 Btu/hr. This is considerably less than the high- 
heat criterion of $40,000 \mathrm{Btu} / \mathrm{hr}$. Tank 241-C-105 will be reclassified from high heat to normal in June 1993.

\section{Occurrence Report}

RL-WHC-TFARM-1993-0044 (OFFNORMAL) - LIOUID OBSERVATION WELL (LOW) ON IANK 241-SX-102 INDICATES INTERSTITIAL LIQUID LEVEL DECREASE (10-DaY Report - May 20, 1993

On April 30, 1993, the quarterly Liquid Observation Well (LOW) reading taken in Tank 241-SX-102 indicated a decrease of 0.2 feet from the established baseline value. The reading was repeated on May 5, 1993, and this reading indicated a decrease of 0.3 feet which was equal to the established decrease criteria. Another reading taken on May 20, 1993, indicated an additional 0.1 -foot decrease. This reading brought the total decrease to 0.4 foot, and exceeded the decrease criteria. On May 20, 1993, Tank 241-SX-102 was declared to be an "assumed leaker." Previous neutron LOW readings have been stable showing minor fluctuation between the baseline and 0.2 -foot decrease. Instrument repeatability is \pm 0.1 foot. The LOW is the primary means of liquid level detection because this tank has a solid surface.

This tank is actively ventilated with a waste temperature of approximately $150^{\circ} \mathrm{F}$. Although decreasing level trends attributed to evaporation have been recorded on other SX Tank Farm tanks ( 1 . e., 105-SX), the 102-SX liquid level has decreased more rapidly than typicaliy observed. 102-SX is a Non-stabilized, Partial Isolated Watch List Tank (Hydrogen list), actively ventilated, with $183 \mathrm{Kgallon}$ drainable liquid.

This event will result in either the need for additional funding/resources or the reprogramming of existing resources from other projects. Plans are being prepared to pump the liquid contents from Tank 241-SX-102 to a double-shell underground storage tank.

4. Tanks Advisory Panel (TAP) Meeting Held May 25-27, 1993 meeting:

The following pages are excerpts from the presentation made at the TAP 


\section{TWRS Problem Statement}

- Remediate tank waste to strive for unrestricted use of the Hanford Site

\section{Why strive for unrestricted use? To not prejudice} future Hanford land use decisions.
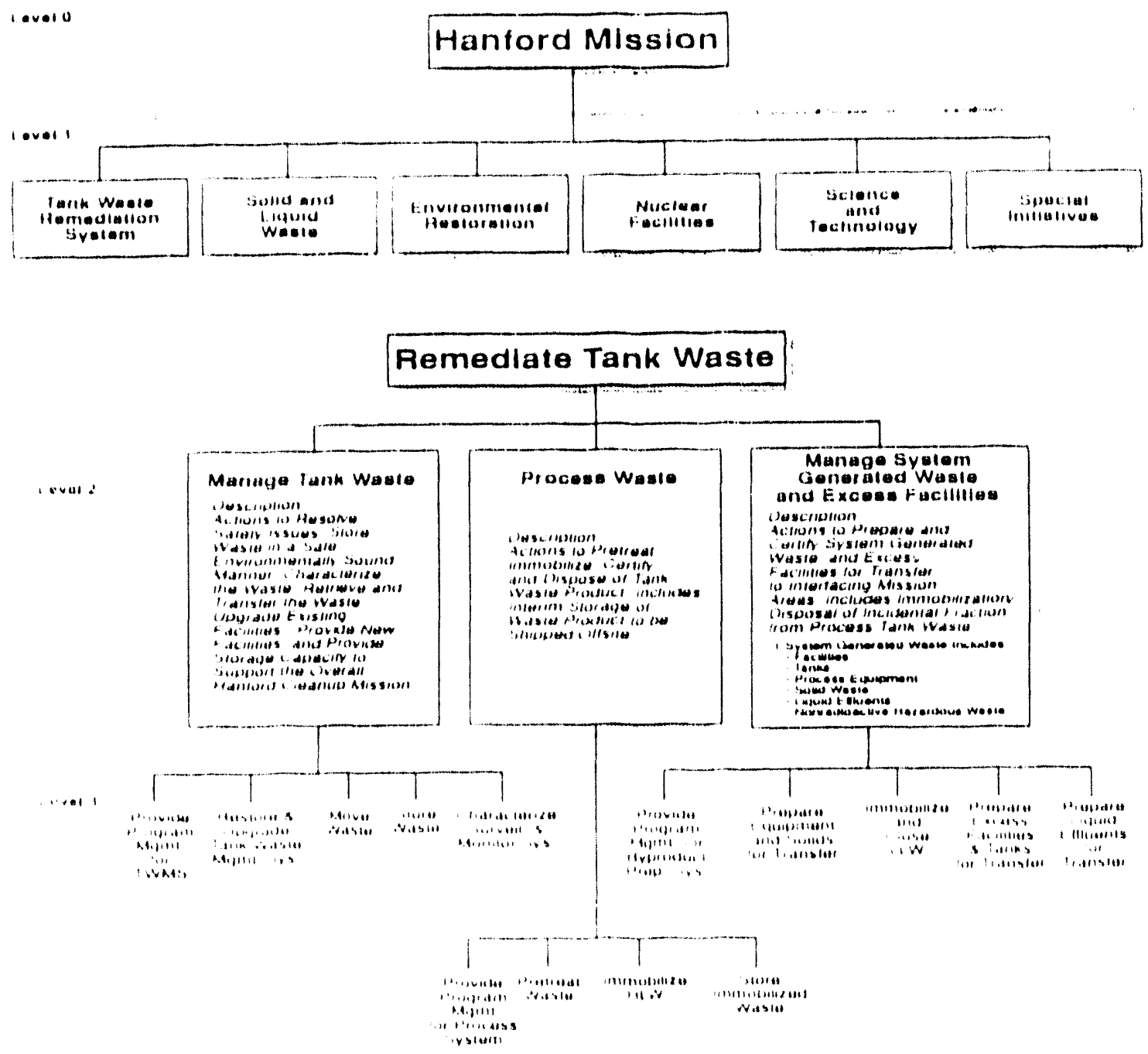


\section{Hanford Defense Waste - Environmental Impact Statement Baseline} Basis for Tri-Party Agreement

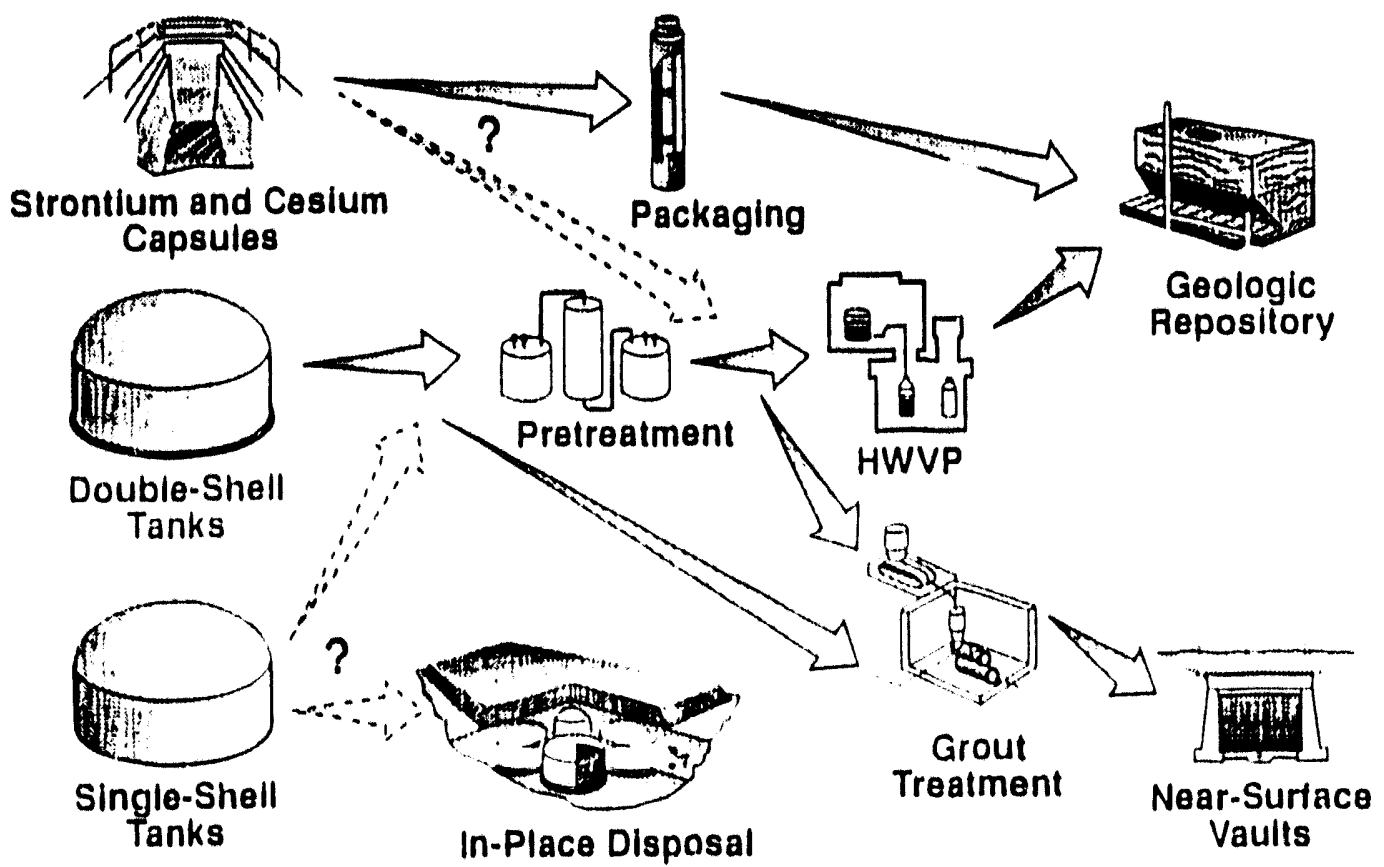

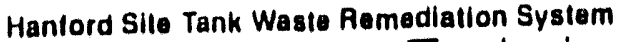

Proposed New Technical Strategy

Technology Development Inve: : nent
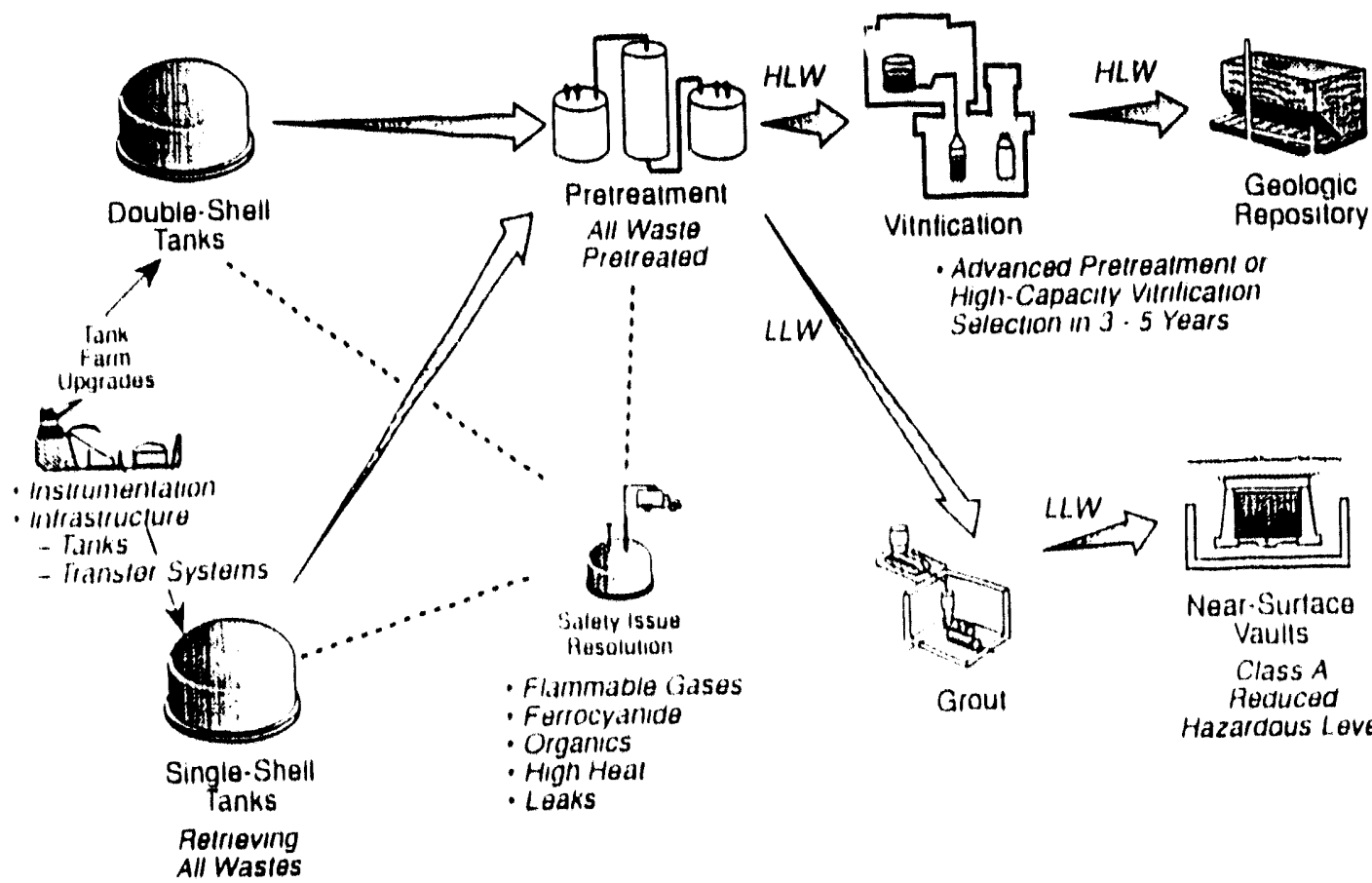

Beducod Hazardous Level 


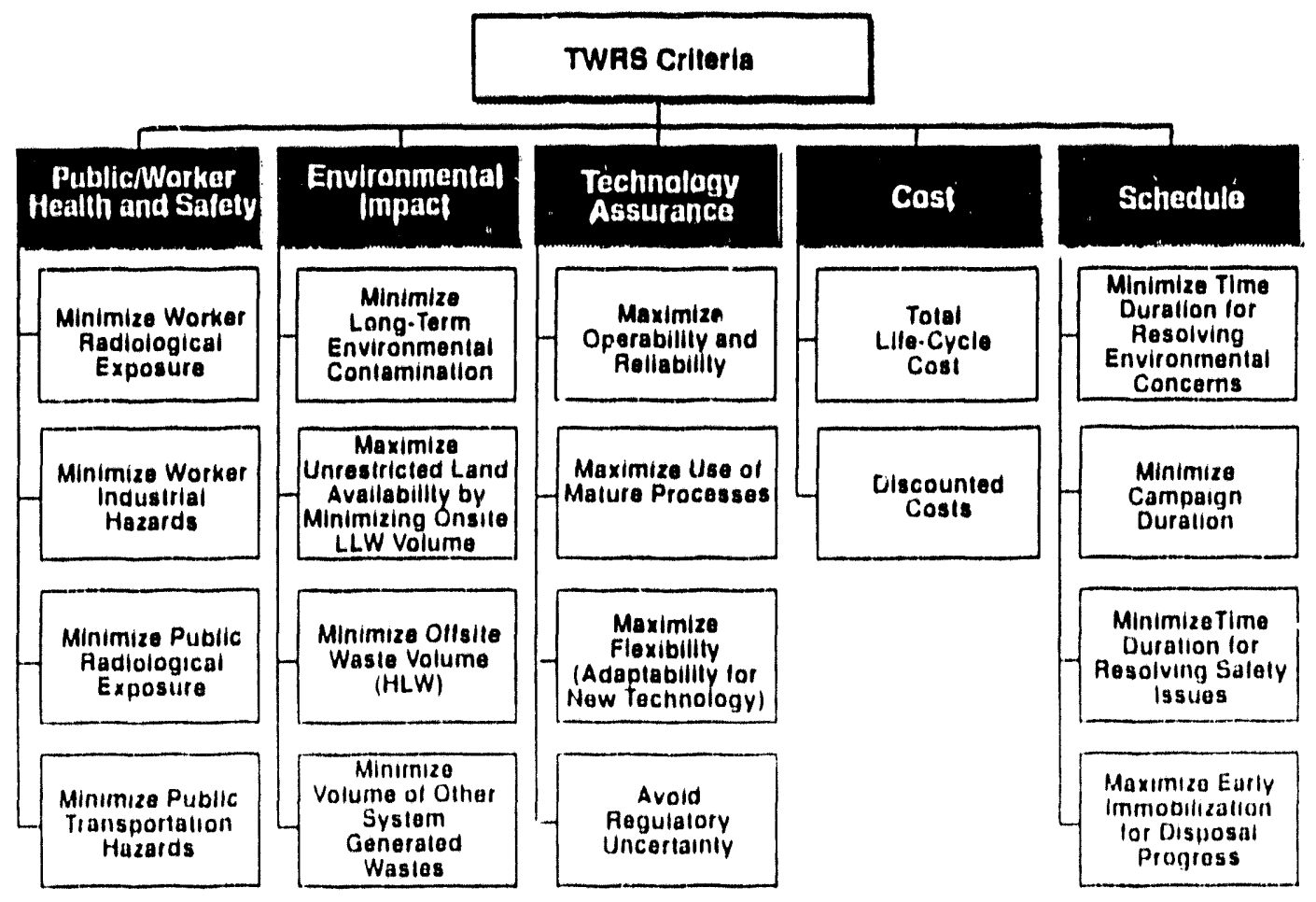

\section{Systems Engineering Process} (System Definition Phase)

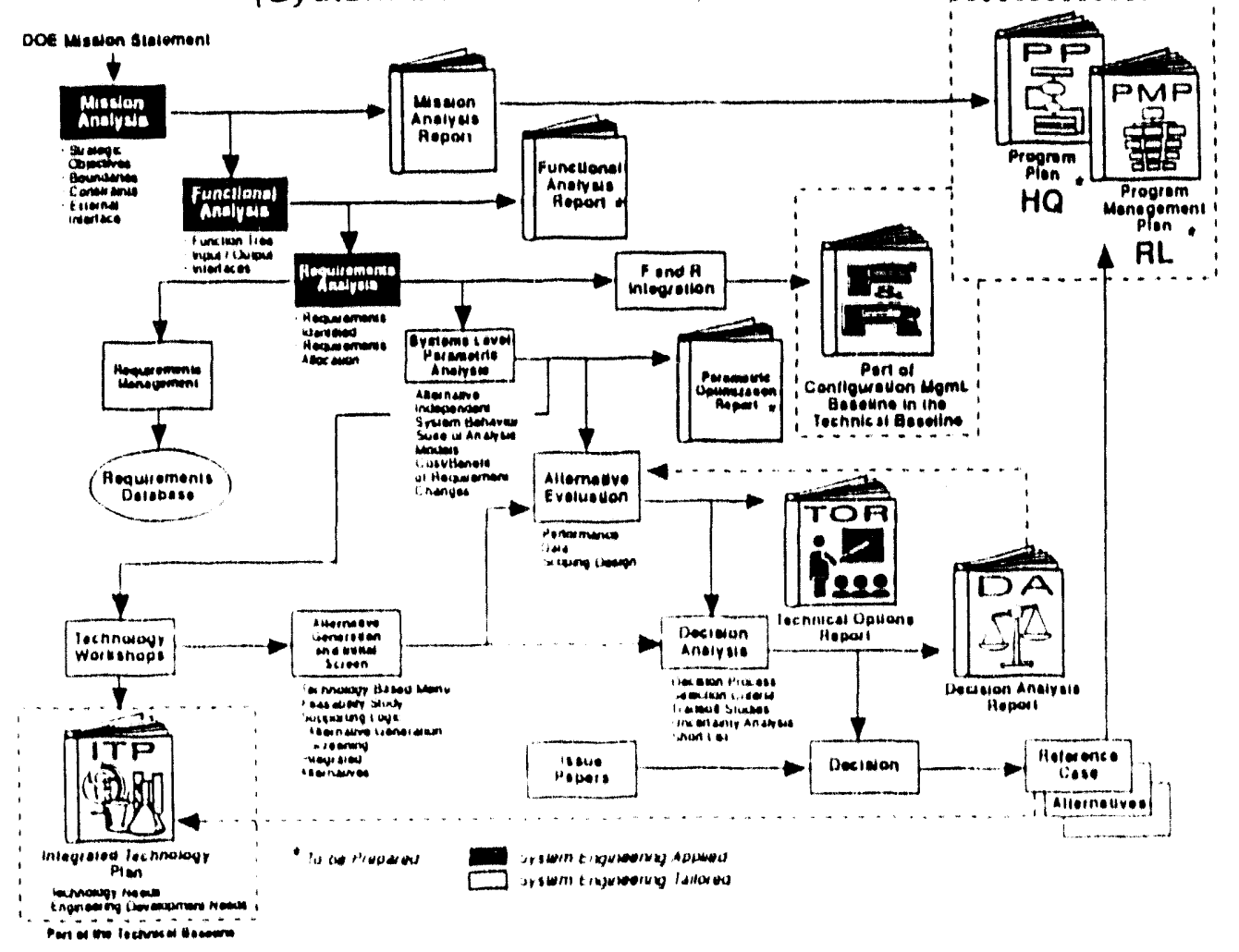


TWRS Level 2 ( $N^{2}$ Dlagram)

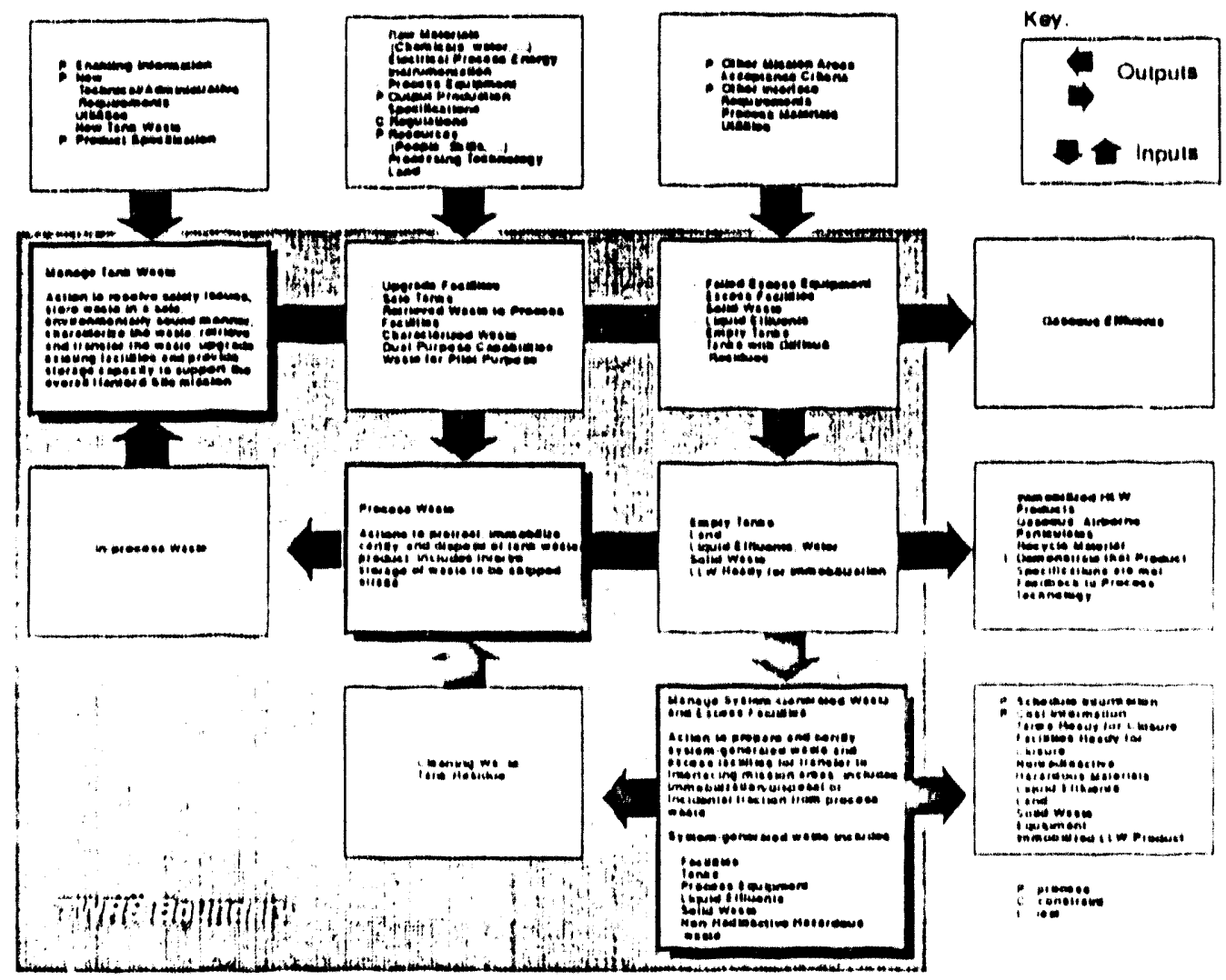

\section{Tank Waste Remediation System Waste Treatment and Disposal Alternatives}

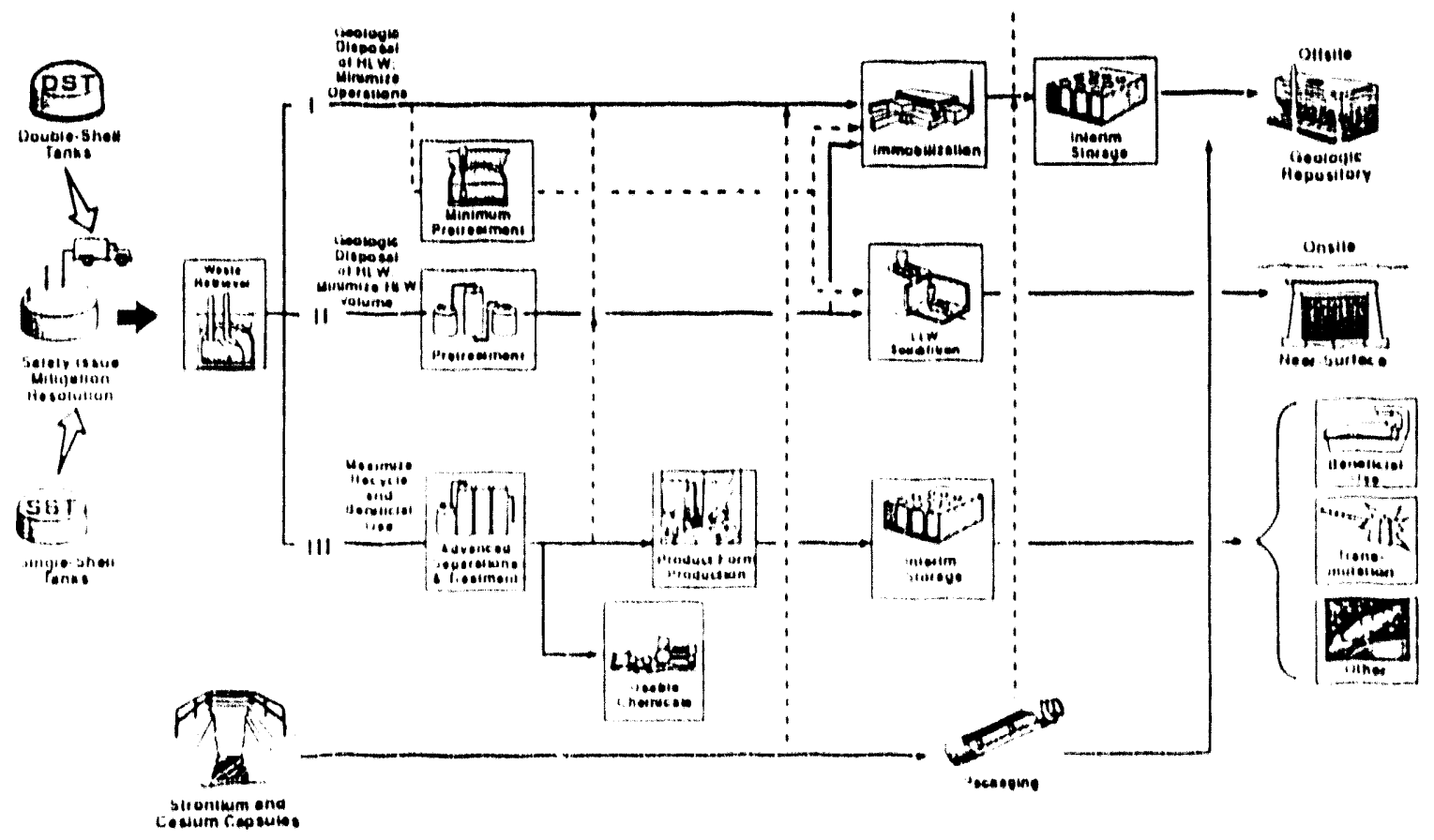




\section{Interim Organic Watch List Tank Criteria:}

- Level I Criterion: TOC $\leq$ A wt.\% (dry basis)

- Level II Criteria:

- TOC $\leq$ B wt. \% (dry basis)

- Moisture $\geq \mathrm{C}$ wt.\%

- Organics concentration mechanisms

- Maximum waste temperature

- Composition tank vapor space gases

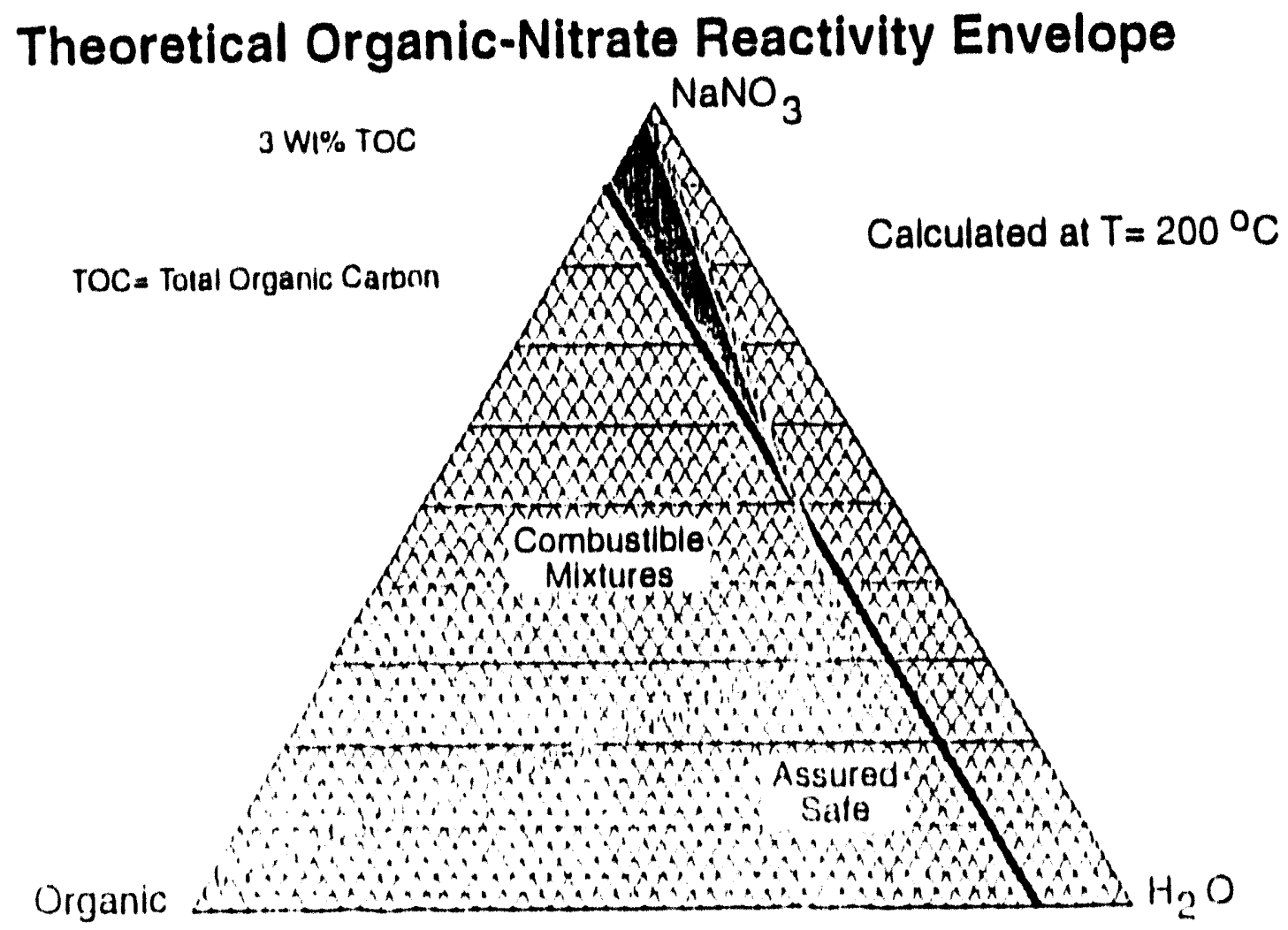


WHC-EP--0182-62
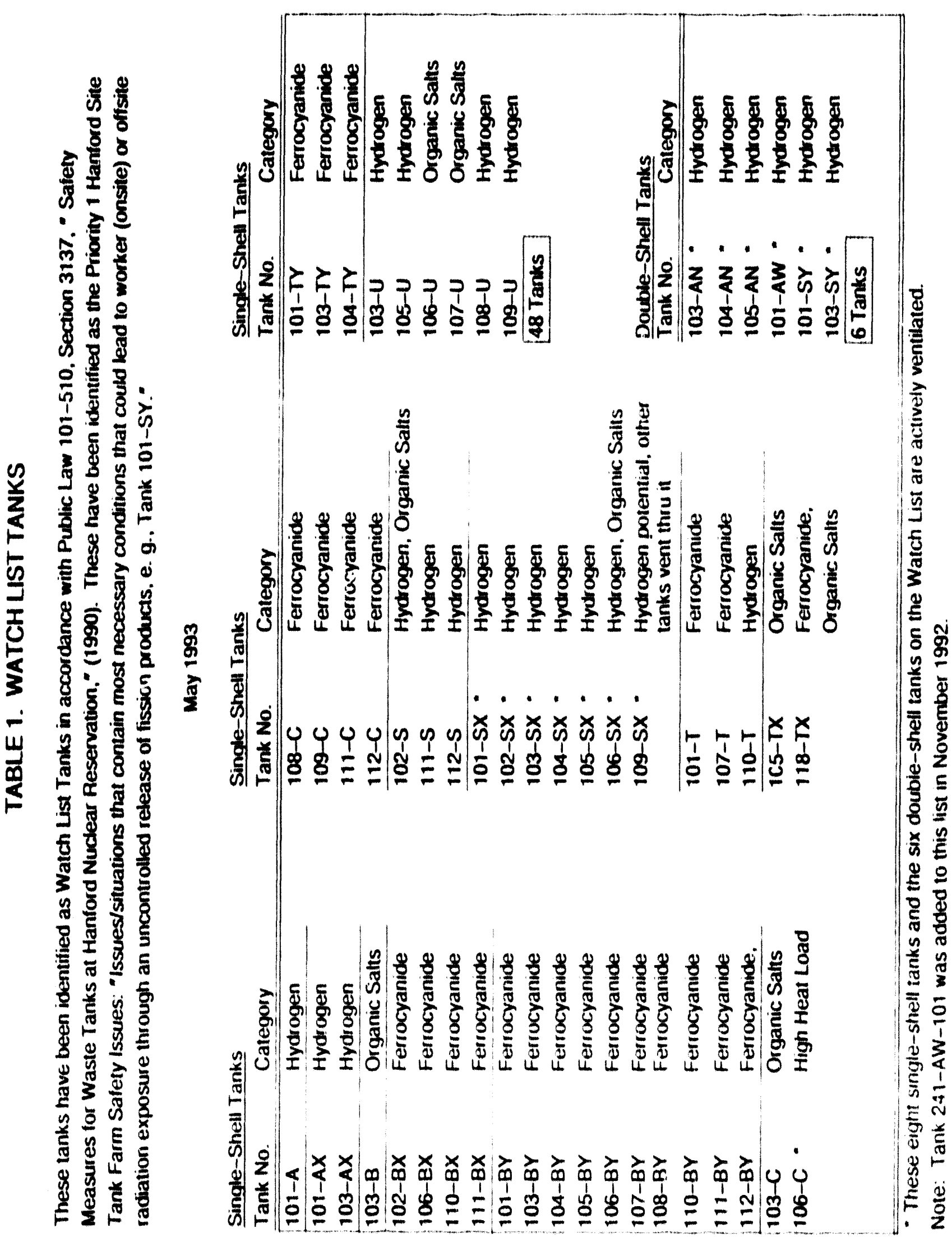
WHC-EP-0182-62

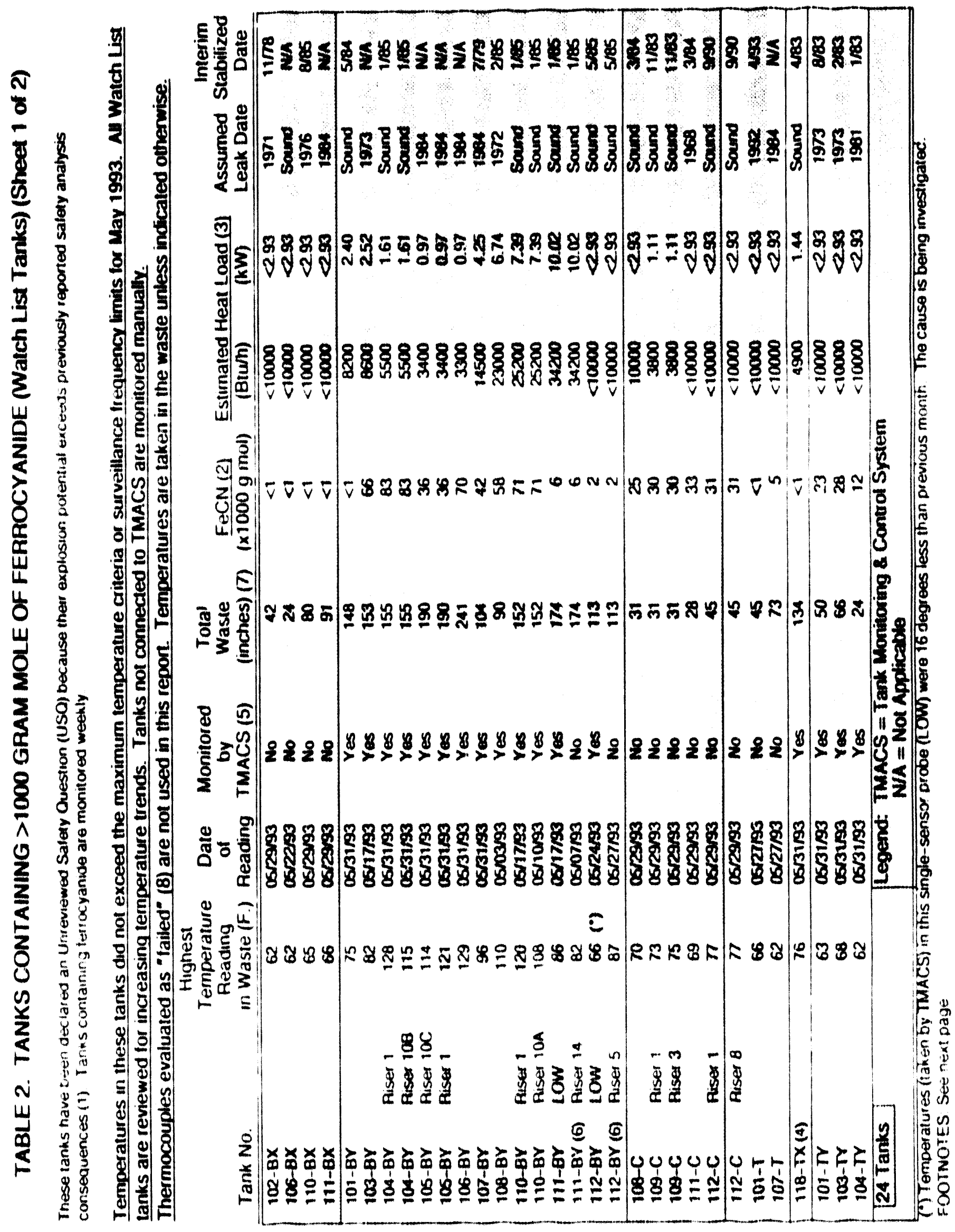


WHC-EP-0182-62

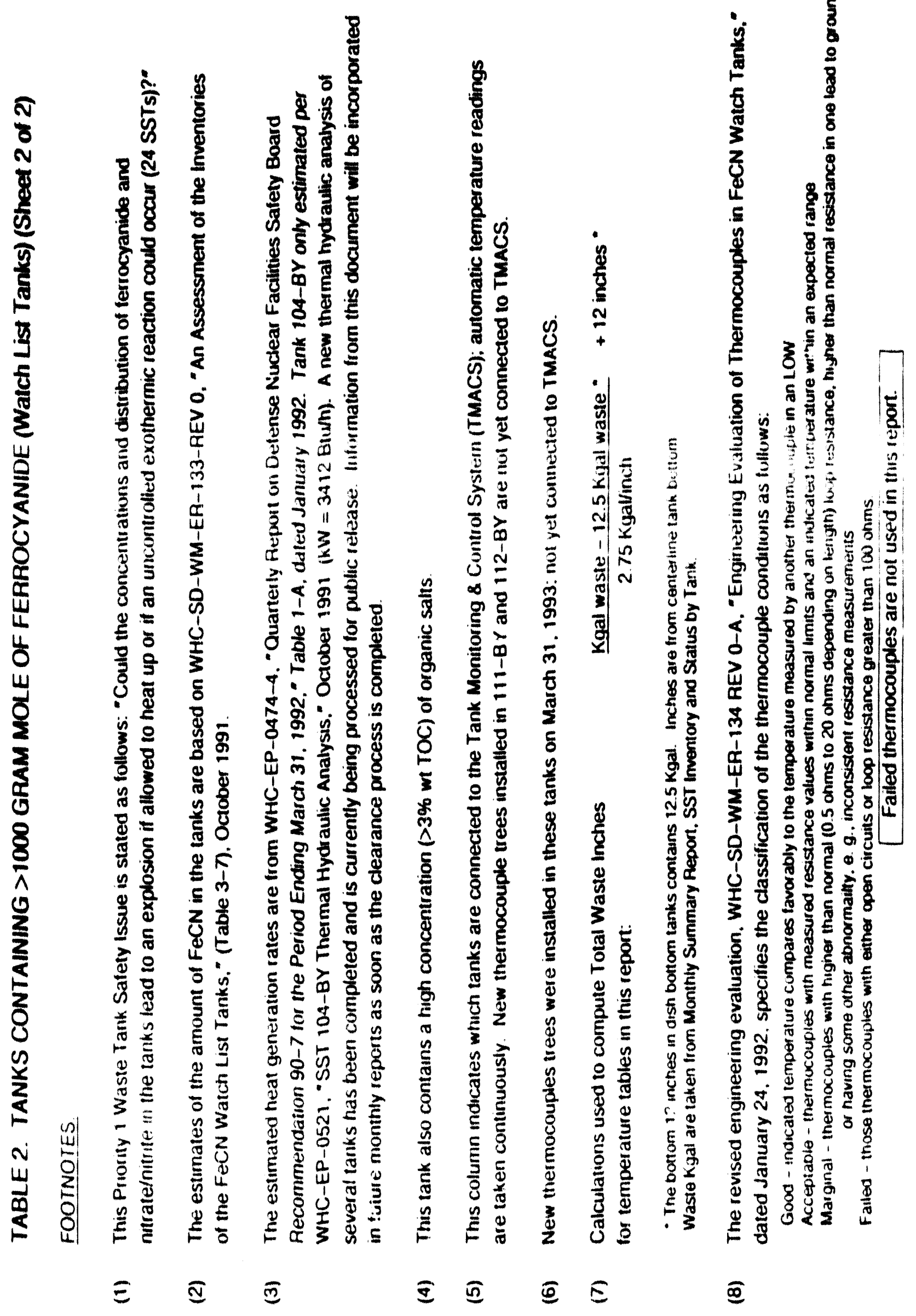



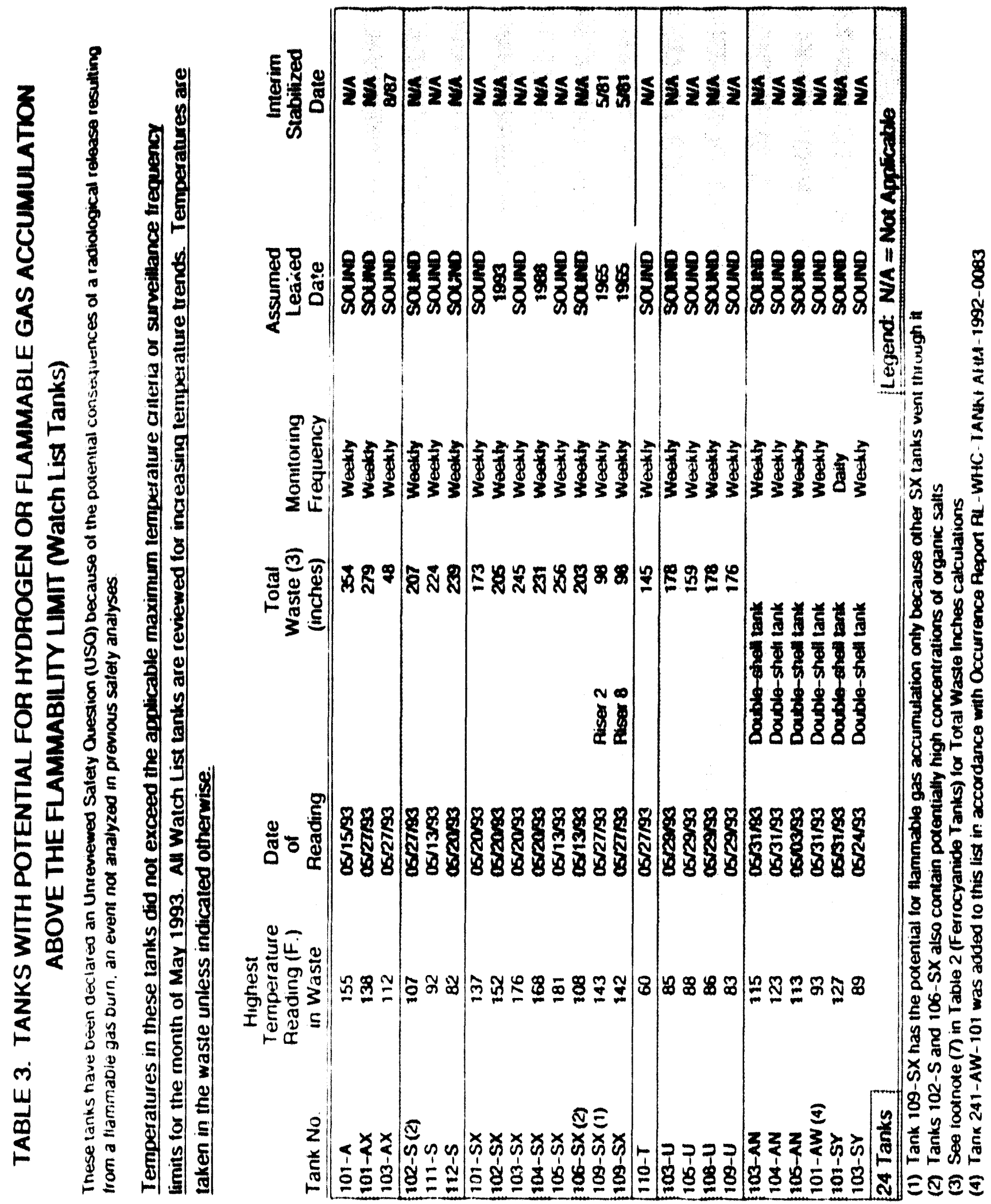
WHC-EP-0182-62
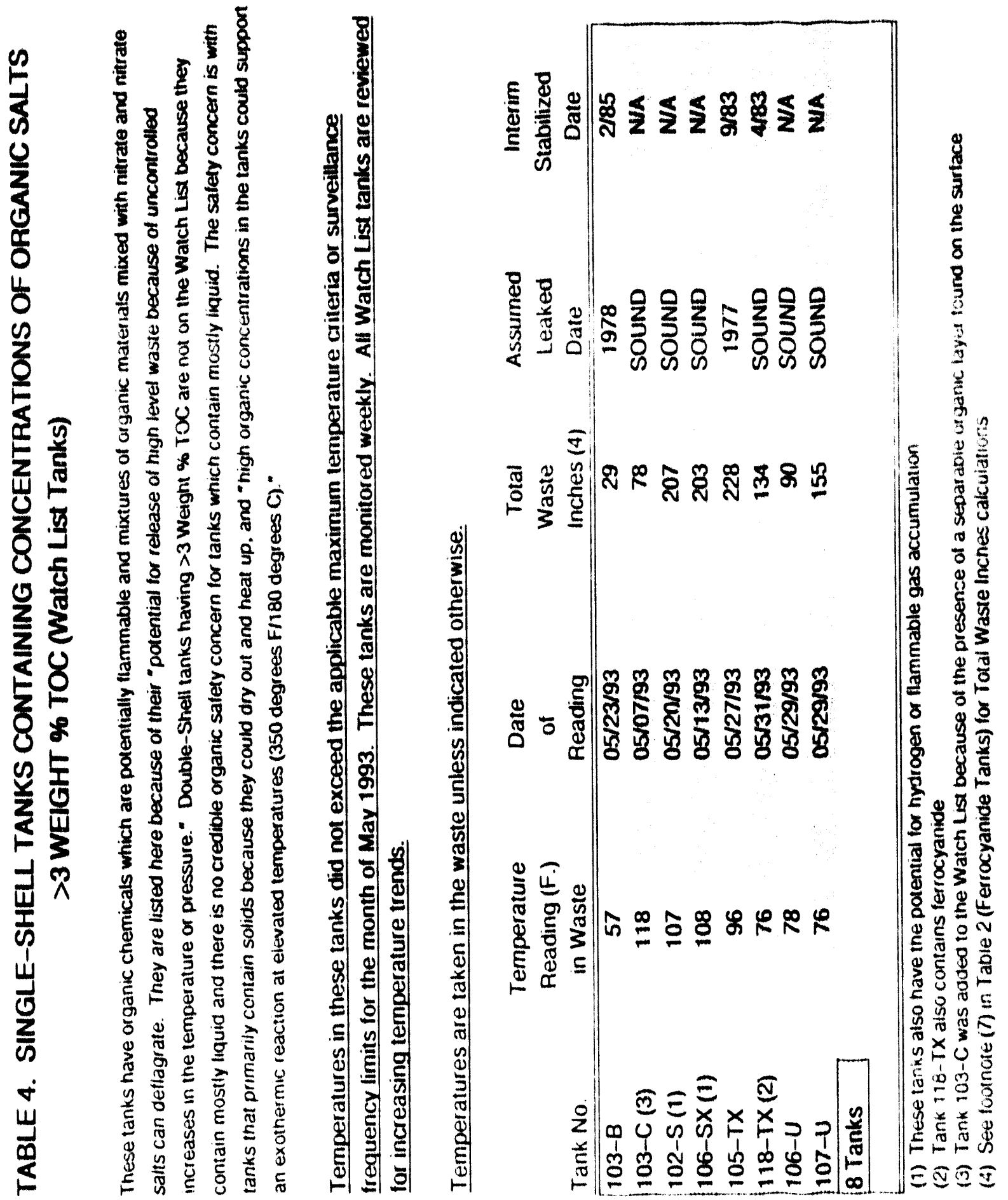


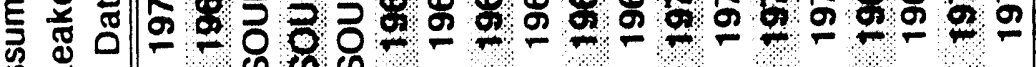
这

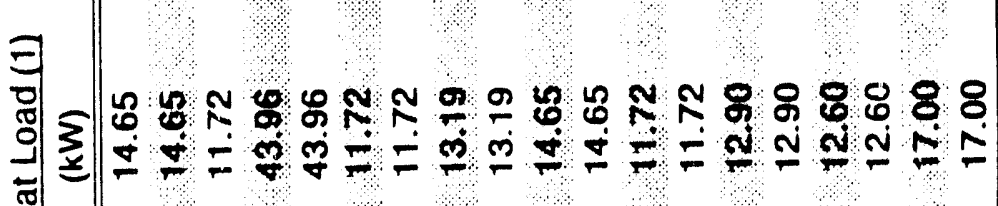

I

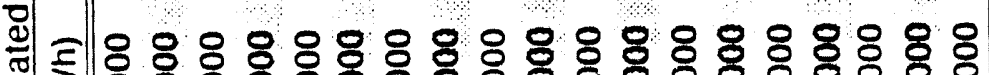

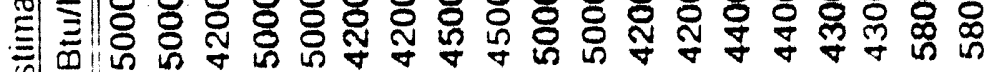

ติ山

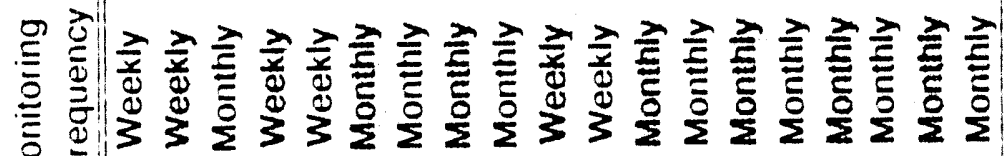

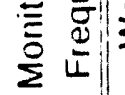

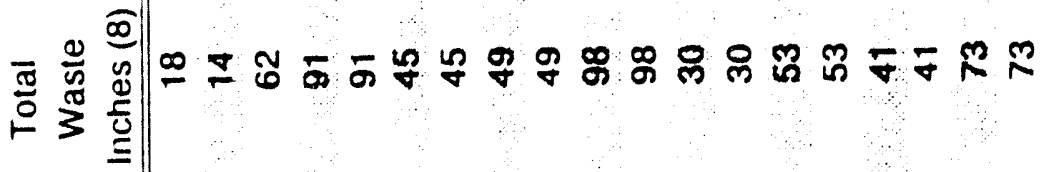

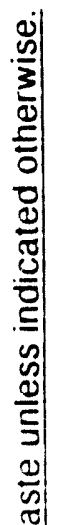

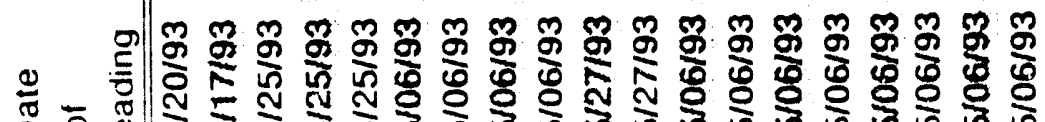

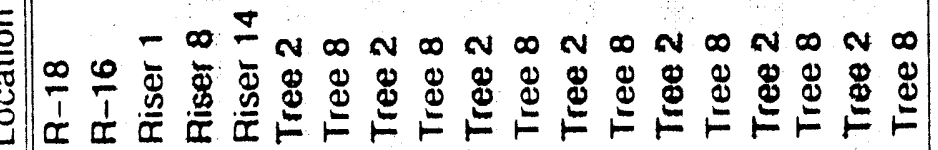

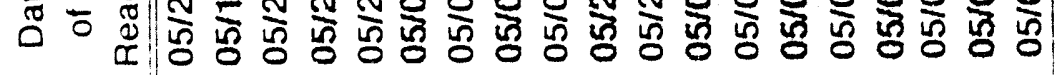

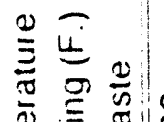

능요 윤

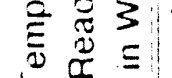

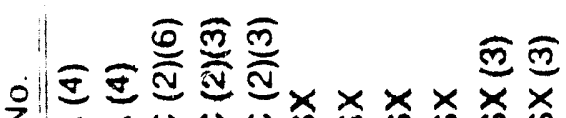

z

妾

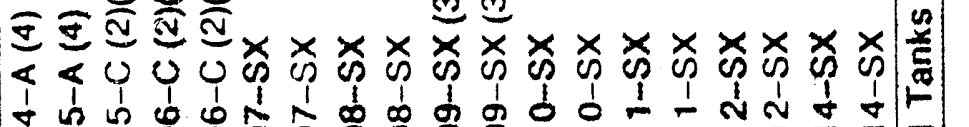




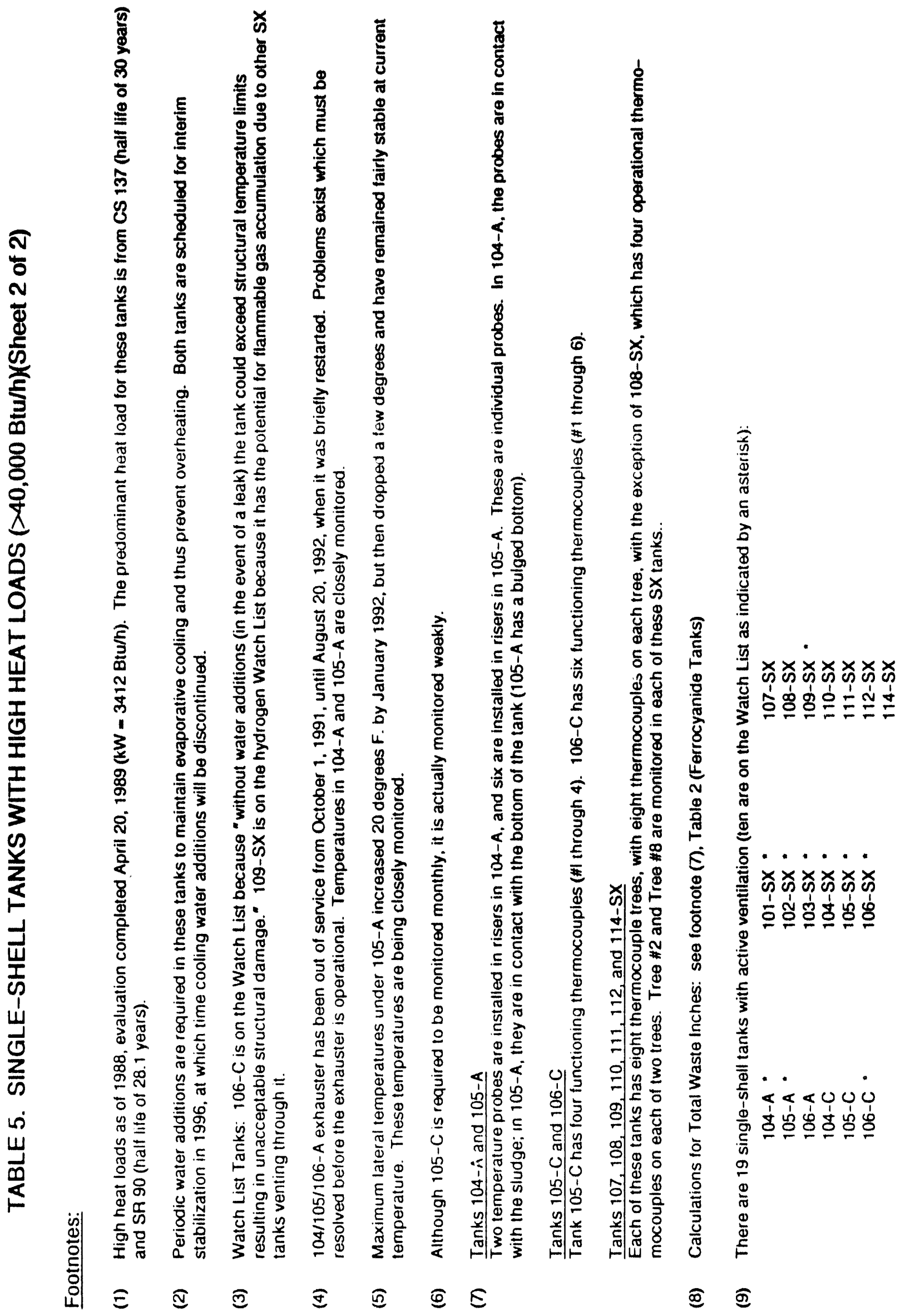


WHC-EP-0182-62

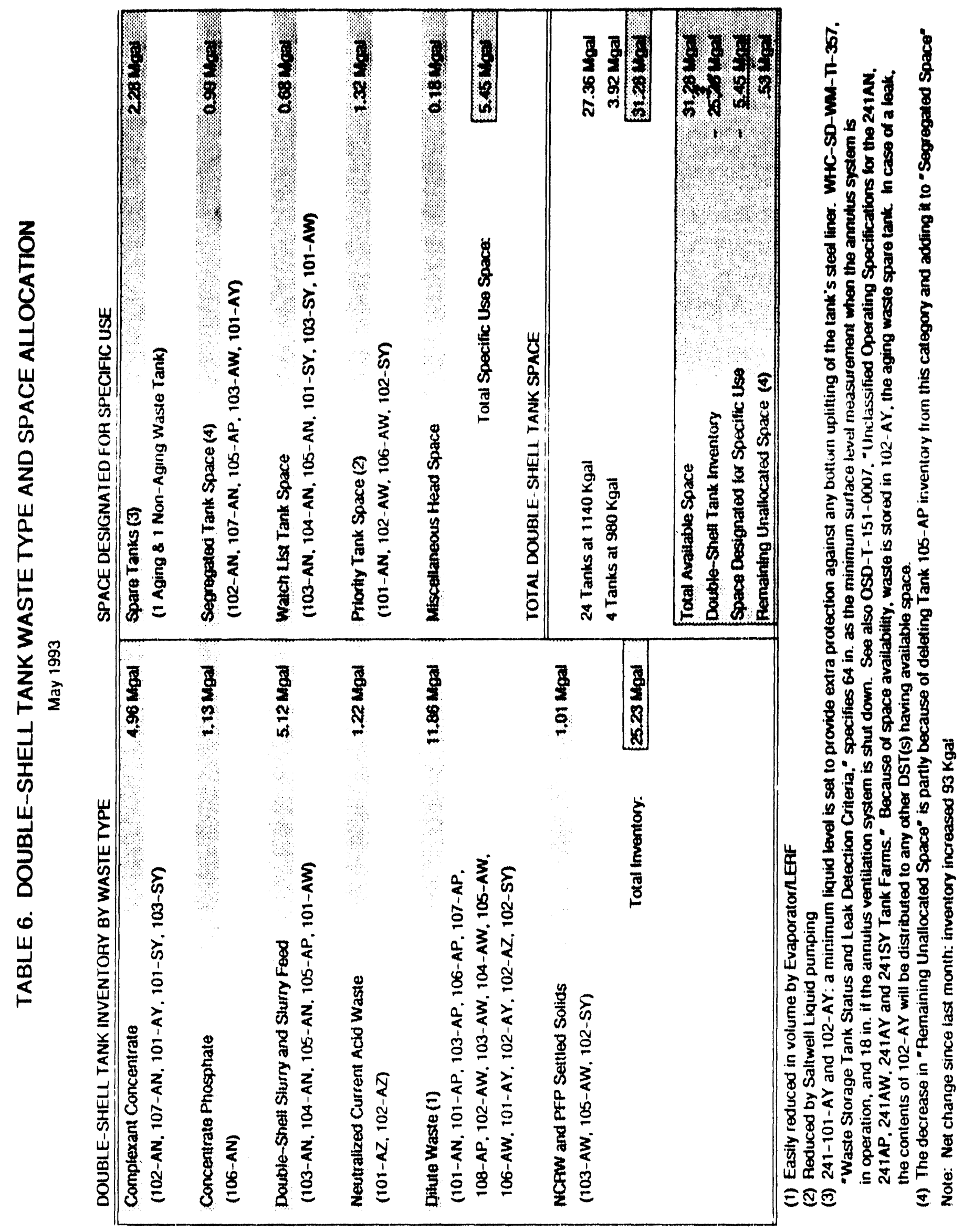




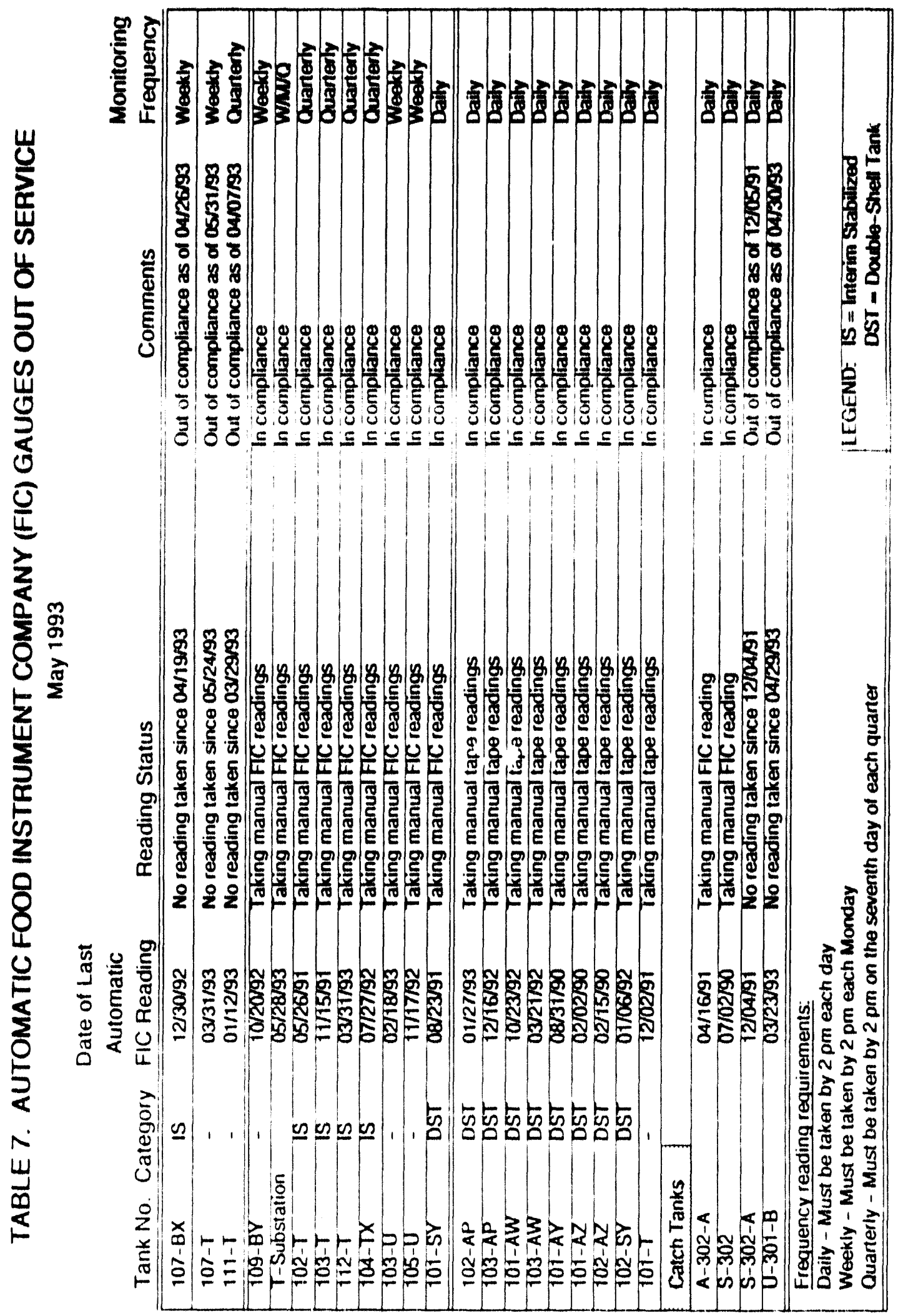




\section{TABLE 8. SINGLE-SHELL TANKS MONITORING COMPLIANCE STATUS 149 TANKS (Sheet 1 of 5)}

The following table indicates whether Single-Shell tank monitoring is in compliance with the requirements as specified in the applicable documents:

NOTE:

All Watch List and High Heat tank temperature monitoring is in compliance.

All Dome Elevation Survey monitoring is in compliance.

All Drywell monitoring is in compliance.

Psychrometrics (2)

In-Iank Pholographs (3)

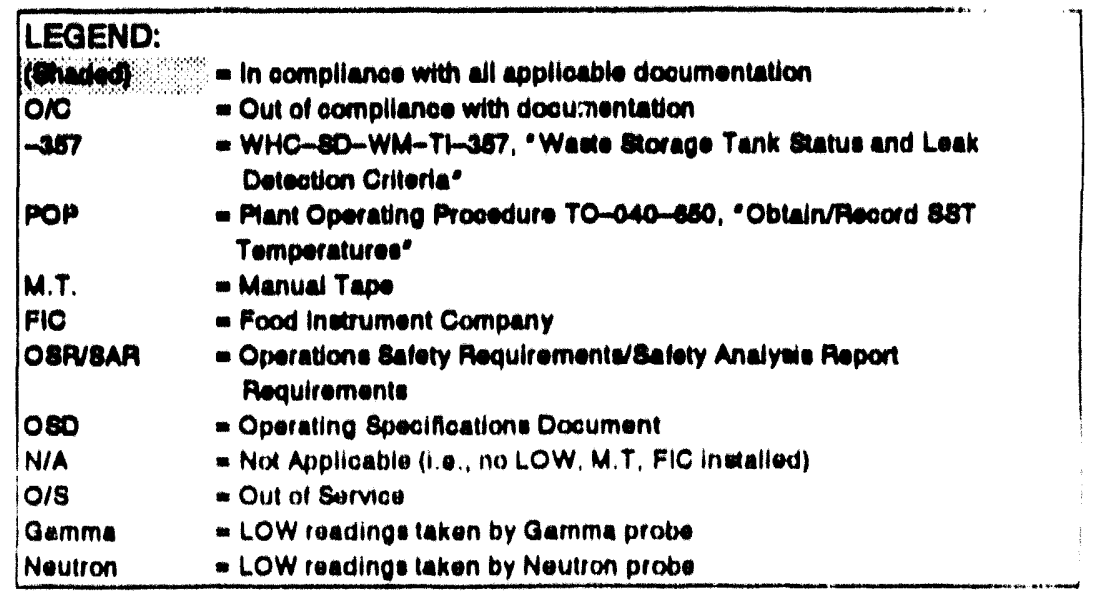

Information as of $5 / 31 / 93$

\begin{tabular}{|c|c|c|c|c|c|c|c|c|c|c|}
\hline \multirow{4}{*}{$\begin{array}{l}\text { Tank } \\
\text { Number }\end{array}$} & \multirow{2}{*}{\multicolumn{2}{|c|}{ Category }} & \multirow{4}{*}{$\begin{array}{l}\text { Temperature } \\
\text { Readings (5) } \\
\end{array}$} & \multirow{3}{*}{\multicolumn{2}{|c|}{$\begin{array}{l}\text { Surface Level } \\
\text { Readings (1) } \\
(-357)\end{array}$}} & \multirow{3}{*}{\multicolumn{2}{|c|}{$\begin{array}{c}\text { LOW Peadings } \\
(-357)\end{array}$}} & \multicolumn{2}{|c|}{ Radiation Readings } & \multirow{4}{*}{$\begin{array}{c}\text { Dome } \\
\text { Elevation } \\
\text { Surveys } \\
\text { (OSRVSAP) }\end{array}$} \\
\hline & & & & & & & & \multirow{3}{*}{$\begin{array}{c}\text { Lateral } \\
\text { Readings } \\
(-357) \\
\end{array}$} & \multirow{3}{*}{$\begin{array}{c}\text { Drywell } \\
\text { Aoadings } \\
\text { (OSFVSAR) }\end{array}$} & \\
\hline & \multirow{2}{*}{$\begin{array}{c}\text { Watch } \\
\text { List }\end{array}$} & \multirow{2}{*}{$\begin{array}{l}\text { High } \\
\text { Heat }\end{array}$} & & & & & & & & \\
\hline & & & & M.T. & FTC & Gamma & Neutron & & & \\
\hline $102-A$ & & & & TMh & & NA & Wh & & & \\
\hline $103-A$ & & & & Wh & & & & & & \\
\hline $104-A$ & & $\bar{x}$ & & O/C & Nh & $T H$ & Wh & & & \\
\hline $101-A x$ & $\bar{x}$ & & & Wh & & & & & & \\
\hline $102-A x$ & & & OIC & & Nh & WA & WA & & & \\
\hline $103-A X$ & $\bar{x}$ & & & $\mathrm{Wh}$ & & WR & Wha & & & \\
\hline 104-Ax & & & & & WA & Wम & TWK & & & \\
\hline $101-8$ & & & & Wh & & Wh & $W h$ & & & \\
\hline $102-8$ & & & & Wh & & N/R & Wh & & & \\
\hline $107-8$ & & & & & WA & NA & Wh & & & \\
\hline $108-8$ & & & & NA & & Nम & NHA & & & \\
\hline $100-8$ & & & & & Wh & N/T & Nh & & & \\
\hline $110-B$ & & & OIC & OIC & MA & NA & NA & & & \\
\hline $111-8$ & & & $O / C$ & साA & & NIA & NIA & & & \\
\hline$\longdiv { 1 2 - 8 }$ & & & & NA & & NA & Wh & & & \\
\hline $201-8$ & & & & & NA & $\mathrm{Wh}$ & Wh & & & \\
\hline $202-8$ & & & & & NA & NIA & NIA & & & \\
\hline $203-8$ & & & & & NA & NसA & NIA & & & \\
\hline $204-8$ & & & & & N/A & NIA & NमA & & & \\
\hline $101-8 x$ & & & $O / C$ & & KIA & Wh & $N / A$ & & & \\
\hline $102-B x$ & $\bar{x}$ & & & & NIA & N/A & NIA & & & \\
\hline $103-3 x$ & & & O/C & NA & & NA & N/A & & & \\
\hline
\end{tabular}


TABLE 8. SINGLE-SHELL TANKS MONITORING COMPLIANCE STATUS 149 TANKS (Sheet 2 of 5 )

Intormation as of 5/31/93

\begin{tabular}{|c|c|c|c|c|c|c|c|c|c|c|}
\hline \multirow{4}{*}{$\begin{array}{l}\text { Tank } \\
\text { Number }\end{array}$} & \multirow{2}{*}{\multicolumn{2}{|c|}{ Cateaory }} & \multirow{4}{*}{$\begin{array}{l}\text { Temperature } \\
\text { Readings (5) }\end{array}$} & \multirow{3}{*}{\multicolumn{2}{|c|}{$\begin{array}{l}\text { Surtace Level } \\
\text { Readinos (1) } \\
(-35 n)\end{array}$}} & \multirow{3}{*}{\multicolumn{2}{|c|}{$\begin{array}{c}\text { LOW Readings } \\
(-357)\end{array}$}} & \multicolumn{2}{|c|}{ Radlation Peadings } & \multirow{4}{*}{$\begin{array}{c}\text { Doria } \\
\text { Elovatin! } \\
\text { Surveys } \\
\text { (OSRVSAH) }\end{array}$} \\
\hline & & & & & & & & \multirow{3}{*}{$\begin{array}{c}\text { Lateral } \\
\text { Readings } \\
(-357)\end{array}$} & \multirow{3}{*}{$\begin{array}{c}\text { Drywell } \\
\text { Peadings } \\
\text { (OSRVSAR) }\end{array}$} & \\
\hline & \multirow{2}{*}{$\begin{array}{c}\text { Watch } \\
\text { List }\end{array}$} & \multirow{2}{*}{$\begin{array}{l}\text { High } \\
\text { Heat }\end{array}$} & & & & & & & & \\
\hline & & & & M.T. & FTC & Gamme & Noutron & & & \\
\hline $108-8 x$ & & & $\partial t \mathrm{C}$ & & $\mathbf{N I N}$ & N/ & Nh & $\mathbf{N A}$ & & \\
\hline $100-8 X$ & & & DाC & $\mathrm{NWI}$ & & $\mathrm{NW}$ & 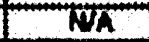 & NI & & \\
\hline $110-8 x$ & $\bar{x}$ & & & & NW & $N /$ & Wh & $N M$ & & \\
\hline $111-8 x$ & $\bar{x}$ & & & & $\mathrm{Mh}$ & & & $\mathrm{H} / \mathrm{W}$ & & \\
\hline $112-8 x$ & & & काट & NII & & Wh & Wh & NW & & \\
\hline $101-6 Y$ & $\bar{X}$ & & & & NIA & & & NIII & & \\
\hline $102-\mathrm{BY}$ & & & $01801 \mathrm{C}$ & & NV & & & N/ & & \\
\hline $103-6 Y$ & $\bar{x}$ & & & & WA & & & NA & & \\
\hline $104-6 Y$ & $x$ & & & & M/A & & & N/R & & \\
\hline $105-B Y$ & $\bar{x}$ & & & & NI & & & NIA & & \\
\hline $108-B Y$ & $x$ & & & & NA & & & NIA & & \\
\hline $107-B Y$ & $x$ & & & & NIA & & & NIA & & \\
\hline $108-B Y$ & $\bar{x}$ & & & & NA & NA & NA & NIA & & \\
\hline $100-3 Y$ & & & $0 / \mathrm{s}-0 / \mathrm{C}$ & $\mathrm{N} / \mathrm{A}$ & & & & $\mathrm{NIA}$ & & \\
\hline $110-B Y$ & $\bar{x}$ & & & & NA & & & NIA & & \\
\hline $111-8 Y$ & $\bar{x}$ & & & & PNA & & & NA & & \\
\hline $112-8 Y$ & $x$ & & & & NA & OIC & O/C & NA & & \\
\hline $101-c$ & & & & & N/A & $N / A$ & WA & NA & & \\
\hline $102-c$ & & & OIC & W/A & & WIA & NDA & Nh & NIA & \\
\hline $103-C$ & $x$ & & & $\mathrm{NA}$ & & NIA & NTI & NA & & \\
\hline $104-\bar{C}$ & & & & NA & & NIA & WA & NMA & & \\
\hline $10 \mathrm{~B}-\mathrm{C}$ & & $\bar{x}$ & & $N A$ & & $N A$ & WA & NIA & & \\
\hline $100-C(1)$ & $x$ & $x$ & & NIA & & NA & $\mathrm{N}$ & NH & & \\
\hline $107-c$ & & & & NA & & NIA & NII & $N / A$ & & \\
\hline $108-C$ & $x$ & & & & $\mathrm{~N} / \mathrm{A}$ & NA & NA & NA & & \\
\hline $109-\mathrm{C}$ & $x$ & & & & N/A & WA & NA & NA & & \\
\hline $110-\mathrm{C}$ & & & & & NT & NA & NDA & Nh & & \\
\hline $111-C$ & $x$ & & & & NIA & NMA & NIA & Wh & & \\
\hline $112-c$ & $\bar{x}$ & & & & NIA & NM & NM & WA & & \\
\hline $201-\bar{C}$ & & & & & NIA & NA & NI & NA & & \\
\hline $202-c$ & & & & & NW & NA & N/A & NA & & \\
\hline $203-C$ & & & & & MA & $N A$ & NA & Tin & & \\
\hline $204-C$ & & & OIC & & $\mathrm{N} / \mathrm{A}$ & NA & NMA & N/A & & \\
\hline $101-5$ & & & OIC & NA & & & & $\mathrm{N}$ & & \\
\hline $102-5$ & $x$ & & & NA & & & & NA & & \\
\hline $103-5$ & & & & NA & & & & $\mathbf{N A}$ & & \\
\hline $104-3$ & & & & & NA & NWA & NA & NAA & & \\
\hline $105-5$ & & & & NA & & & & NA & & \\
\hline $108-5$ & & & & $N / A$ & & & & NA & & \\
\hline $107-5$ & & & & $N A$ & & $\mathrm{NA}$ & NIA & $N / A$ & & \\
\hline $108-\mathrm{S}$ & & & & & & & & N/A & & \\
\hline $108-5$ & & & & $\mathrm{~N} / \mathrm{A}$ & & & & NAA & & \\
\hline $110-5$ & & & & NA & & & & NA & & \\
\hline $111-5$ & $x$ & & & $\mathrm{NIA}$ & & & & NIA & & \\
\hline $112-\mathrm{s}$ & $x$ & & & NA & & & & NIA & & \\
\hline $101-5 x$ & $\bar{x}$ & & & $N A$ & & & & $N / A$ & & \\
\hline $102-5 x$ & $x$ & & & $N / A$ & & & & NA & & \\
\hline $103-5 x$ & $\bar{x}$ & & & $\mathrm{~N} / \mathrm{A}$ & & & & $\mathrm{NiA}$ & & \\
\hline $104-5 x$ & $x$ & & & NIA & & $0 / \mathrm{s}-0 / \mathrm{C}$ & $0 / 5.01 \mathrm{C}$ & $N / A$ & & \\
\hline $105-5 x$ & $x$ & & & NIA & & & & $0 / C$ & & \\
\hline $106-5 x$ & $x$ & & & N/A & & & & NA & & \\
\hline $107-\$ x$ & & $x$ & & & $N / A$ & $\mathrm{NIA}$ & NIA & $\mathrm{OLC}$ & & \\
\hline $108-5 x$ & & $x$ & & & $N / A$ & NA & NA & OIC & & \\
\hline
\end{tabular}




\section{TABLE 8. SINGLE-SHELL TANKS MONITORING COMPLIANCE STATUS}

\section{TANKS (Sheet 3 of 5)}

Information as of 5/31/93

\begin{tabular}{|c|c|c|c|c|c|c|c|c|c|c|}
\hline \multirow{4}{*}{$\begin{array}{l}\text { Tank } \\
\text { Number }\end{array}$} & \multirow{2}{*}{\multicolumn{2}{|c|}{ Catogory }} & \multirow{4}{*}{$\begin{array}{l}\text { Temperature } \\
\text { Readings (5) }\end{array}$} & \multirow{3}{*}{\multicolumn{2}{|c|}{$\begin{array}{l}\text { Surface Levol } \\
\text { Peadings (1) } \\
(-35 n)\end{array}$}} & \multirow{3}{*}{\multicolumn{2}{|c|}{$\begin{array}{c}\text { LOW Readings } \\
(-357)\end{array}$}} & \multicolumn{2}{|c|}{ Aadiation Readings } & \multirow{4}{*}{$\begin{array}{c}\text { Dorine } \\
\text { Elevation } \\
\text { Surveys } \\
\text { (OSPVSAI3) }\end{array}$} \\
\hline & & & & & & & & \multirow{3}{*}{$\begin{array}{c}\text { Lateral } \\
\text { Peadings } \\
(-35 n) \\
\end{array}$} & \multirow{3}{*}{$\begin{array}{c}\text { Drvwell } \\
\text { Readings } \\
\text { (OSPVSAR) }\end{array}$} & \\
\hline & \multirow{2}{*}{$\begin{array}{l}\text { Watch } \\
\text { L/st }\end{array}$} & \multirow{2}{*}{$\begin{array}{l}\text { High } \\
\text { Heat }\end{array}$} & & & & & & & & \\
\hline & & & & M.T. & FTC & Gamma & Noutron & & & \\
\hline $110-8 x$ & & $x$ & & & $\mathrm{~W}$ & $N$ & WR & $\pi$ & & \\
\hline $111-8 x$ & & $\bar{x}$ & & & Nh & Wh & WA & $O / C$ & & \\
\hline $112-8 x$ & & $x$ & & & Tw/ & Non & Nom & OIC & & \\
\hline $115-5 x$ & & & OIS-OIC & & Non & W/A & N/A & O/C & & \\
\hline nI-T & $\bar{x}$ & & & & 018 & NAK & $N A$ & Wh & & \\
\hline $102-7$ & & & $018-0 / C$ & NIS & & Wh & NTK & $\mathbb{N} / \mathrm{A}$ & & \\
\hline $103-7$ & & & & Wh & & $N / A$ & $\mathrm{Wh}$ & $N 2$ & & \\
\hline $104-7$ & & & & & NIA & & & NIA & & \\
\hline $10 \mathbf{5}-\mathrm{T}$ & & & $0 / \mathrm{s}-0 / \mathrm{C}$ & $\mathrm{NIA}$ & & $\mathrm{NiA}$ & $\mathrm{NiA}$ & ina & & \\
\hline $110-1$ & $x$ & & & NIA & & & & N/A & & \\
\hline $111-1$ & & & & $\mathrm{NiA}$ & OIC & & & $\mathrm{NIA}$ & & \\
\hline $112-\mathrm{T}$ & & & & N/A & & NA & NIA & NA & & \\
\hline $201-T$ & & & & & $\mathrm{~W} / \mathrm{h}$ & NA & $\mathrm{Nin}$ & $\mathrm{NA}$ & & \\
\hline $202-7$ & & & & & $N \pi$ & NA & N/A & NA & & \\
\hline $203-1$ & & & & & NA & $\mathrm{NH}$ & NA & $N A$ & & \\
\hline $204-T$ & & & & & NWI & Wh & N/K & NIA & & \\
\hline $101-T X$ & & & $O / 8$ - O/C & NIA & & Tia & $\mathrm{K} K \mathrm{~K}$ & $\mathrm{~W} / \mathrm{K}$ & & \\
\hline $102-1 x$ & 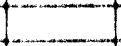 & 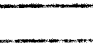 & & & N/A & & & NA & & \\
\hline $103-1 x$ & & & & $N A$ & & Wh & Wh & $\mathrm{N} / \mathrm{h}$ & & \\
\hline $104-1 x$ & & & & N/A & & NA & NIA & N/n & & \\
\hline $106-1 x$ & $\bar{x}$ & & & & $\mathrm{Nh}$ & $01 \mathrm{~B}-01 \mathrm{C}$ & $018-0 i \mathrm{C}$ & TWA & & \\
\hline $106-1 x$ & & & & & NA & & & N/A & & \\
\hline $115-1 x$ & & & & & NA & & & $\mathrm{NiA}$ & & \\
\hline $116-7 x$ & & 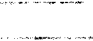 & OIS OIC & & N/A & N/A & N/A & $N A$ & & \\
\hline $111-1 x$ & & & O/C & & $\mathrm{N} / \mathrm{A}$ & & & Nh & & \\
\hline $118.1 x$ & $x$ & & & NIA & & & & N/A & & \\
\hline $101 \ldots T Y$ & $x$ & & 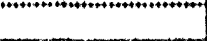 & NiA & & NiA & $\mathrm{N} / \mathrm{A}$ & $\mathrm{NIA}$ & & \\
\hline $102-1 Y$ & 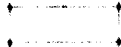 & & & $N / A$ & & NIA & N/A & NIA & & \\
\hline $1037 Y$ & $x$ & & & NIA & & & & $\mathrm{NiA}$ & & $m+1$ \\
\hline $104-1 Y$ & $x$ & 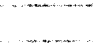 & & N/A & & NIA & NIA & NIA & & \\
\hline $105-T Y$ & $\ldots$ & & & & $\mathrm{NiA}$ & $\mathrm{Nin}$ & $\mathrm{NiA}$ & $\mathrm{NiA}$ & & \\
\hline $106-1 Y$ & 4 & & & & N/A & N/A & N/A & NIA & & \\
\hline $101-0$ & & & Uis & & $\mathrm{NiA}$ & NiA & $N A$ & $\mathrm{~N} / \mathrm{A}$ & & \\
\hline $102-4$ & & & & NIA & & & & NA & & \\
\hline 103.4 & $x$ & & 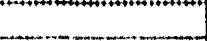 & $\mathrm{N} / \mathrm{A}$ & & & & NIA & & \\
\hline $104-U$ & & & $0 / 5-0 / C$ & & NIA & N/A & N/A & NA & & \\
\hline $105-0$ & $x$ & & & NiA & & & & $\mathrm{Nin}$ & & 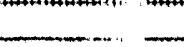 \\
\hline $106-U$ & $x$ & & & NIA & & & & NIA & & \\
\hline
\end{tabular}


TABLE 8. SINGLE-SHELL TANKS MONITORING COMPLIANCE STATUS 149 TANKS (Sheet 4 of 5)

Intormation as of 5/31/93

\begin{tabular}{|c|c|c|c|c|c|c|c|c|c|c|}
\hline \multirow{4}{*}{$\begin{array}{l}\text { Tank } \\
\text { Number }\end{array}$} & \multirow{2}{*}{\multicolumn{2}{|c|}{ Category }} & \multirow{4}{*}{$\begin{array}{l}\text { Temperafure } \\
\text { Ruadings (5) }\end{array}$} & \multirow{3}{*}{\multicolumn{2}{|c|}{$\begin{array}{l}\text { Surtace Levol } \\
\text { Aoadings (1) } \\
(-357)\end{array}$}} & \multirow{3}{*}{\multicolumn{2}{|c|}{$\begin{array}{c}\text { LOW Aesdings } \\
(-357)\end{array}$}} & \multicolumn{2}{|c|}{ Aadiaflon headinges } & \multirow{4}{*}{$\begin{array}{l}\text { Dome } \\
\text { Elovation } \\
\text { Survoy: } \\
\text { (OSPSAl) }\end{array}$} \\
\hline & & & & & & & & \multirow{3}{*}{$\begin{array}{c}\text { Lateral } \\
\text { Readings } \\
(-357)\end{array}$} & \multirow{3}{*}{$\begin{array}{c}\text { Drvwell } \\
\text { Racilings } \\
\text { (OBPJSAA) }\end{array}$} & \\
\hline & \multirow{2}{*}{$\begin{array}{l}\text { Watch } \\
\text { List }\end{array}$} & \multirow{2}{*}{$\begin{array}{l}\text { Hlon } \\
\text { Heal }\end{array}$} & & & & & & & & \\
\hline & & & & M.T. & FIC & Gamma & Noutron & & & \\
\hline $107-U$ & $\bar{x}$ & & & NIA & & & & Wh & & \\
\hline $108-U$ & $\bar{x}$ & & & NWK & & & & Wh & & \\
\hline 1000 & $\bar{x}$ & & & Wh & & & & $\mathrm{W}$ & & \\
\hline $110-0$ & & & & Th & & $\mathbf{M A}$ & Wh & TWh & & \\
\hline $111-0$ & & & & KIA & & & & $\mathrm{W} / \mathrm{K}$ & & \\
\hline $112-U$ & & & & & WI & NW & Wh & Wh & & \\
\hline $201-0$ & & & Oाट & & ThK & 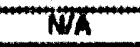 & $W h$ & $\mathrm{~N} / \mathrm{K}$ & & \\
\hline $202-U$ & & & & & TWh & Wh & WR & Wh & & \\
\hline $203-0$ & & & orc & & $\mathrm{Wh}$ & Wh & NI & Wh & & \\
\hline $201-0$ & & & ठट & & WWA & THA & NA & N/A & & \\
\hline
\end{tabular}

Catch Tanks and Special Surveillance Facililies

\begin{tabular}{|c|c|c|c|c|c|c|c|c|c|c|}
\hline$A-302-A$ & $N / A$ & $N / A$ & N/A & NIA & & NIA & N/A & NIA & TIA & $\mathrm{N} / \mathrm{A}$ \\
\hline$A-302-6$ & $N / A$ & N/A & NIA & & NA & N/A & NIA & NIA & NIA & NIA \\
\hline $311-E A$ & N/A & NIA & $N / A$ & NA & & $N / A$ & NIA & NIA & NIA & $N / A$ \\
\hline $152-A \bar{x}$ & $N / A$ & NIA & NIA & & NIA & & & & & \\
\hline$1 5 \longdiv { - A 2 }$ & NIA & $N I A$ & NIA & NIA & & NA & NIA & NIA & NIA & TIA \\
\hline $154-A 2$ & NIA & $N / A$ & NIA & & NIT & NIA & NIA & NI & NIA & WA \\
\hline BX-TKSMP & $N / A$ & NIA & N/A & & NA & & & & & \\
\hline A-TKVBMP & NIA & N/A & NA & & NIA & & & & & \\
\hline 204-AA & $N / A$ & NIA & NA & & & & & & & \\
\hline $417-4$ & NIA & NIA & Wh & & & & & & & \\
\hline Vont sia. & $N / A$ & NIA & N/A & & NA & NIA & NA & NA & NWA & NM \\
\hline $8-302$ & N/A & NIA & Wh & NIA & & NIA & NMA & N/ & NMA & NA \\
\hline $5-302-A$ & NIA & NIA & NA & Nh & OIC & $\mathrm{NA}$ & WA & NIA & Nh & TVI \\
\hline $5-304$ & N/A & N/A & N/A & & NI & N/A & NA & NA & NA & WA \\
\hline$r x-302-8$ & NIA & N/A & NI & OIC & NA & NIA & NIA & $N /$ & TW & $\mathbf{N}$ \\
\hline$x-302-c$ & NIA & NIA & NMA & NIA & & NA & Wha & $\mathbf{N A}$ & NA & MN \\
\hline$U-301-B$ & N/A & NIA & $\mathrm{NA}$ & NA & OIC & WA & $\mathrm{NA}$ & NA & Wh & $\mathrm{WA}$ \\
\hline$U x-302-A$ & NIA & NIA & NA & NA & & $\mathrm{N} / \mathrm{A}$ & N/A & TIA & $N / A$ & NA \\
\hline $141-5$ & $N / A$ & N/A & NIA & & NA & NIA & NIA & $\mathrm{NTA}$ & NMA & $N A$ \\
\hline $142-3$ & N/A & NIA & Na & & WA & NA & NA & $\mathbf{N A}$ & NII & NA \\
\hline \multirow[t]{2}{*}{$\begin{array}{l}\text { Totale: } \\
149 \operatorname{tanke}\end{array}$} & \multirow{2}{*}{$\begin{array}{c}48 \\
\text { Watch } \\
\text { Un } \\
\text { Tanke } \\
\text { (4) }\end{array}$} & \multirow{2}{*}{$\begin{array}{c}11 \\
\text { High } \\
\text { Hoat } \\
\text { Tanks } \\
\text { (4) }\end{array}$} & \multirow{2}{*}{$\begin{array}{l}\text { Olc: } \\
20 \text { ianke - } \\
\text { (momiannual } \\
\text { monitoring } \\
\text { hequency) (5) }\end{array}$} & \multirow[t]{2}{*}{$\begin{array}{l}\text { O/C } \\
0 \text { tanke } \\
\text { I calch Iank }\end{array}$} & \multirow[t]{2}{*}{$\begin{array}{l}O / C \\
3 \text { tanke } \\
2 \text { calch Ianks }\end{array}$} & $\begin{array}{l}O / C \\
3 \text { tanke }\end{array}$ & $\begin{array}{l}O / C \\
3 \text { tanke }\end{array}$ & \multirow[t]{2}{*}{$\begin{array}{l}\text { O/C } \\
\text { o tanke }\end{array}$} & \multirow[t]{2}{*}{0} & \multirow[t]{2}{*}{0} \\
\hline & & & & & & $\begin{array}{r}50 \operatorname{tank} \\
12 \mathrm{a}\end{array}$ & $\begin{array}{l}\text { LOWO } \\
\text { 8) }\end{array}$ & & & \\
\hline
\end{tabular}

\section{Footnotes:}

(1) All SSTs have oither manual lape or FIC, with the excoplion of 108-S and 101-T, which have both. Tank $101-T$ also has a if cord. All SST FICs are connecled lo CASS, with the exceplion of 106-Bx; however, the connection tor many lanks is broks: For such cases, manual readings are laken. Manual surface lovel readings include readings taken by manual tape, manual FIC (not connected to Computer Automated Surveillance Systern, 106-BX), manual readings of automatic FIC (il CASS is printing " $D^{*}$ ), or automatic FIC. In some casos, the surlace level readings are taken using a zip cord. While less accurate, such readings are accoplable lor me日ting the surlace level reading requirements.

(2) Psychrometric readings are to be taken on tanks with active exhausters (High heat tanks 104/105-A, 105/106-C, 107, 108, 109, 110,111, 112, and 114-SX). Psychrometrics were not laken from 9/91 10 9/92. Exhauster down on 104/105-A; no readings being taken. 105/106-C psychrometric readings are required to be taken on a monthly frequency. Readings were taken in high heat SX lanks in March 1993. Psychrometic trequency not specilied in - 357 or operating procedures.

(3) In-lank Pholographs are not required by -357. In-lank photographs were not taken belween 1991 and 1993.

(4) Two tanks are on both category lists (106-C, 109- SX) 
TABLE 8. SINGLE-SHELL TANKS MONITORING COMPLIANCE STATUS 149 TANKS (Sheet 5 of 5 )

\section{Foolnotes continued:}

(5) Temperature readinge may be regulated by OSD, -357 , or POP. Addilionally, hloh heat load lanke are regulated by OSFSAR. Thermcouples in the 0 lanke deaignated OVS-OC are oul of service; there are eliher no thermocouple probes in inese lanks, of probes have been cut off, covered over, or are otherwise unable lo lunction. The 080 doee nol require readinges or repait of out-ol-service thermocouples lor the 82 non-heat load $(240,000$ Blu/hr) tanks. However, operating procedure (POP) requiree that aftempts are 10 be made eemiannually in January and July to obtain readings from these tanks. In March 1993, ceven additional TX Iank Iemperature readinge were oblained by measuring resistance uaing the wall of the pipe as ground. Temperatures were comparable lo last lemperalures laken in 1983.29 lank: are O/C because elther no allempls were made lo read them January - March or readinges could not be oblained. 


\section{TABLE 9. DOUBLE-8HELL TANKS MONITORING COMPLIANCE 8TATUS 28 TANK8}

The following table indicates whether Double-shell tank monitoring is in compliance with the requirements as epecified in the appliceble documents:

Note:

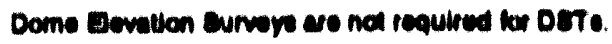

Popahrometrites (l)

In-lank Phocogrephe (a)

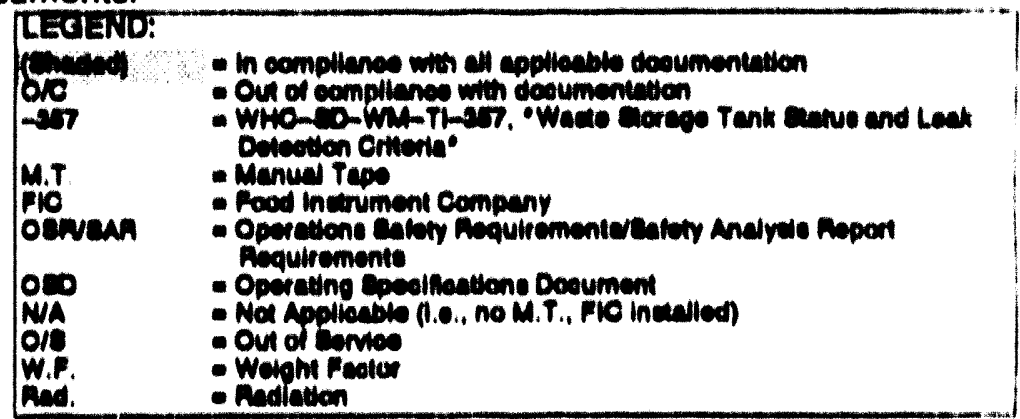

Intormation es of 8/31/93

\begin{tabular}{|c|c|c|c|c|c|c|c|}
\hline \multirow{3}{*}{$\begin{array}{l}\text { Tank } \\
\text { Number }\end{array}$} & \multirow[b]{3}{*}{ Watch Liat } & \multirow{3}{*}{$\begin{array}{c}\text { Temperalure } \\
\text { Aeadines } \\
(4) \\
\text { (OSD) }\end{array}$} & \multirow{2}{*}{\multicolumn{2}{|c|}{$\begin{array}{l}\text { Surfuce Level } \\
\text { Readinge (1) } \\
\text { (-357, OSPVAR) }\end{array}$}} & \multicolumn{3}{|c|}{ Acdiation Resdines } \\
\hline & & & & & \multicolumn{2}{|c|}{$\begin{array}{c}\text { Leak Detcolion } \\
\text { Plts (6) } \\
\text { (-387, OsPysAR) }\end{array}$} & \multirow{2}{*}{$\begin{array}{l}\text { Annulun } \\
(-357)\end{array}$} \\
\hline & & & M.T. & TrTe & & मad. & \\
\hline 101-AN & & & N/A & & & & \\
\hline 102-AN & & & Nh & & & J\% & \\
\hline TOS-AN & $x$ & & NWh & & & & రाट \\
\hline TO4-AN & $\mathbf{x}$ & & TIR & & & orc & \\
\hline TOL-AN & $\bar{x}$ & & NWh & & & & \\
\hline TOSAN & & & $\mathrm{N} / \mathrm{T}$ & & & & \\
\hline $107=A N$ & & & WWI & & & & \\
\hline \multicolumn{8}{|l|}{ TO1-A } \\
\hline \multicolumn{8}{|l|}{$102-A$} \\
\hline TOJ-A & & & & का & & oro & \\
\hline 106-1: & & & OII & & & OKe & \\
\hline \multicolumn{8}{|l|}{$106-A$} \\
\hline $100-A$ & & OK & & & & oro & \\
\hline $107-\lambda$ & & oke & & & & ore & \\
\hline $106-x^{2}$ & & OK & & & & OK & \\
\hline 101-AW & $\bar{X}$ & & & Oा5 & & & \\
\hline \multicolumn{8}{|l|}{ DOE-AW } \\
\hline TOEAW & & & & का & & & \\
\hline \multicolumn{8}{|l|}{$104-A W$} \\
\hline TOLAW & & & & 하 & & & \\
\hline \multicolumn{8}{|l|}{ TOCAW } \\
\hline TOT-AY & & & & का & & & $\sigma \%$ \\
\hline $102-A Y$ & & & & & & OKe & $8 \%$ \\
\hline 10t-n & & & & ㅇ․ & & काठ' & \\
\hline $102-\lambda 2$ & & & & ठा5 & & & \\
\hline 101-sY & $x$ & & & & & & \\
\hline $102-8 Y$ & & & & 018 & & & \\
\hline $103-87$ & $x$ & & & & & & \\
\hline $\begin{array}{l}\text { Towale: } \\
28 \text { lenks }\end{array}$ & Walch Lin Tanko & $\begin{array}{l}\text { ON: } \\
\text { (14) }\end{array}$ & $0 / C$ & $\begin{array}{l}\text { O/C } \\
0\end{array}$ & ore: & $\begin{array}{l}\text { O/C. } \\
12 \text { tanke }\end{array}$ & $\begin{array}{l}\text { O/C: } \\
\text { J lanke }\end{array}$ \\
\hline
\end{tabular}

(1) All DSTs have bolh manual tape and FIC, with ine exceplion of the AN Tank Farm which only has FICs. The manual lape is used when Ihe FIC is oul of service. O/C will be shown when no readings are oblained.

(2) Psychromelric readings are taken on DSTs. All DSTs are equinped with active exhausters. Psychromelrics were nol taken lrom 9/91 lo 9/92: readings on some lanks were resumed beginning Oclober 1992. Frequency of psychrometric moniloring is not specilied in -357 or operaling procedures.

(3) In-lank photographs are not required by -357. Last in-lank pholographs in DSTs were taken April 1989.

(4) OSD requires DST temperaturea to be laken weokly. Tank 101-SY temperatures are oblained shiftwise with increased readings laken prior to and lollowing gas venting. Four AP lanks are O/C because the transmilting equipment is O/S.

(5) Failure of both leak detection systems requires repair of at least one syatem within 5 working days. Fallure of one syatem only, repair must be within 10 working days. Per -357. If repair of out-ol-service syetems exceeds inese ilmetrames, systems are O/C. OUl-ol-service systems which have not exceeded inese timelrames will be shown as $0 / 8$.

- Although data is being received lor 101-AZ, il is considered questlonable. 
WHC-EP-0182-62

\section{APPENDIX A}

TANK AND EQUIPMENT CODE AND STATUS DEFINITIONS

$A=i$ 
WHC-EP-0182-62

This page intentionally left blank 


\section{TANK AND EQUIPMENT CODE/STATUS DEFINITIONS}

\section{May 1993}

\section{IANK STATUS GODES}

\section{HASTE TYPE}

$\begin{array}{ll}\text { AGING } & \text { Aging Waste (Neutralized Current Acid Waste [NCAW]) } \\ C C & \text { Complexant Concentrate Waste } \\ C P & \text { Concentrated Phosphate Waste } \\ D C & \text { Dilute Complexed Waste } \\ \text { DN } & \text { Dllute Non-Complexed Waste } \\ \text { OSS } & \text { Double-Shell Slurry } \\ \text { OSSF } & \text { Double-Shell Slurry Feed } \\ \text { NCPLX } & \text { Non-Complexed Waste } \\ \text { PD/PN } & \text { Plutonium-Uranium Extraction (PUREX) Neutralized Cladding } \\ & \text { Removal Waste (NCRW) transuranic Waste (TRU) } \\ P T & \text { Plutonium Finishing Plant (PFP) TRU Solids }\end{array}$

IANK USE (DOUBLE-SHELL TANKS ONLY)

CWHT Concentrated Waste Holding Tank

DRCVR Dllute Receiver Tank

EVFD Evaporate Feed Tank

GRTFD Grout Feed Tank

SRCVR Slurry Recelver Tank

2. SOLID AND LIQUID VOLUME DETERMINATION METHOOS

F Food Instrument Company (FIC) Automatic Surface Level Gauge

$M \quad$ Manual Tape Surface Level Gauge

P Photo Evaluation

S Sludge Level Measurement Device

3. DEEINITIONS

WASTE TANKS - GENERAL

Waste Tank

Safety lisue

Watch: List Tank
A potentially unsafe condition in the handing of waste material in underground storage tanks that requires corrective action to reduce or eliminate the unsafe condition

An underground storage tank containing waste that requires special safety precautions because it may have serious potential for release of high level radioactive waste because of uncontrolled increases in temperature or pressure. Special restrictions have been placed on these tanks by "Safety Measures for Waste Tanks at Hanford Nuclear Reservation," Section 3137 of the National Defense Authorization Act or Fiscal rear 1991. November 5, 1990. Public Law 101-510. (also known as the Wyden Amendment). 
WASTE TYPES

Aging Waste (ÁGING)

Concentrated Complexant (CC)

Concentrated Phosphate Waste (CP)

Dllute Complexed Waste (DC)
High level, first cycle solvent extraction waste from the PUREX plant (NCAW)

Concentrated product from the evaporation of dilute complexed waste.

Waste originating from the decontamination of $100 \mathrm{~N}$ Area reactor. Concentration of this waste produces concentrated phosphate waste.

Characterized by a high content of organic carbon including organic complexants:

ethylenediaminetetra-acetic acid (EDTA), citric acid, hydroxyethyl-ethylenediaminetriacetic acid (HEDTA), and iminodiacetate (IDA) being the major complexants used. Main sources of DC waste in the OST system are saltwell liquid inventory.

Dilute Non-Complexed Waste (DN)

Low activity liquid waste originating from $T$ and $S$ Plants, the 300 and 400 Areas, PUREX facllity (decladding supernatant and miscellaneous wastes), $100 \mathrm{~N}$ Area (sulfate waste), B Plant, saltwells, and PFP (supernate).

Double-Shell

Slurry (DSS)

Double-shell

slurry Feed (DSSF)

Non-complexed

(NCPLX)

PUREX Decladding

$(P D / P N)$

PFP TRU Solids

(PT)

Drainable

Interstitial

Liquid (DIL)
Waste that exceeds the sodium aluminate saturation boundary in the evaporator without exceeding receiver tank composition limits. For reporting purposes, DSS is considered a solid.

Waste concentrated just before reaching the sodium aluminate saturation boundary in the evaporator without exceeding receiver tank composition limits. This form is not as concentrated as DSS.

General waste term applied to all Hanford site liquors not identified as complexed.

PUREX Neutralized Cladding Removal Waste (NCRW) is the solids portion of the PUREX plant neutralized cladding removal waste stream; received in Tank Farms as a slurry. NCRW solids are classified as transuranic (TRU) waste.

TRU solids fraction from PFP PIant operations.

Interstitial liquid that is not held in place by capillary forces, and will therefore migrate or move by gravity. 
Supernate

Ferrocyanide

WASTE STATUS

In-Service Tank

Out-of-Service

Tank
The liquid above the solids in waste storage tanks.

A compound of iron and cyanide commonly expressed as FeCN. The actual formula for the ferrocyanide anion is $\left[\mathrm{Fe}(\mathrm{CN})_{6}\right]^{-4}$.

The waste classification of a tank being used, or planned for use, for the storage of liquid (in excess of a minimum supernatant liquid heel) in conjunction with production and/or waste processing.

A tank which does not meet the definition of an inservice tank. Before September 1988, these tanks were defined as inactive in this report. [Note: All single-shell tanks (SST) are out of service.]

STABILIZATION (Single-Shell Tanks only)

Interim

Stabilized

(IS)
A tank which contains less than $50,000 \mathrm{gal}$ of drainable interstitial liquid and less than $5,000 \mathrm{gal}$ of supernatant liquid. If the tank was jet pumped to achieve interim stabilization, then the jet pump flow must also have been at or below $0.05 \mathrm{gpm}$ before interim stabilization criteria is met.

\section{ISOLATION (Single-Shell Tanks only)}

Partially

Interim Isolated

(PI)

Interim Isolated

(II)

\section{TANK INTEGRITY}

Sound

Assumed Leaker

Assumed Re-Leaker
The administrative designation reflecting the completion of the physical effort required for Interim Isolation except for isolation of risers and piping that is required for jet pumping or for other methods of stabilization.

The administrative designation reflecting the completion of the physical effort required to minimize the addition of liquids into an inactive storage tank, process vault, sump, catch tank, or diversion box.

The integrity classification of a waste storage tank for which surveillance data indicate no loss of

liquid attributed to a breach of integrity.

The integrity classification of a waste storage tank for which surveillance data indicate a loss of

liquid attributed to a breach of integrity.

A condition that exists after a tank has been declared as an "assumed leaker" and then the 
survelllance data indicates a new loss of liquid attributed to a breach of integrity.

TANK INVESTIGATION

Intrusion

A term used to describe the infiltration of liquid into a waste tank.

\section{SURVEILLANCE INSTRUMENTATION}

Drywells

Laterals

Surface Levels

Automatic FIC
Drywells are vertical boreholes with 6-in. (internal diameter) carbon steel casings positioned radially around SSTs. Periodic monitoring is done by gamma radiation or neutron sensors to obtain scan proffles of radiation or moisture in the soll as a function of well depth, which could be indicative of tank leakage. These wells range between 50 and $250 \mathrm{ft}$ in depth, and are monitored between the range of 50 to $150 \mathrm{ft}$. The wells are sealed when not in use. They are called drywells because they do not penetrate to the water table and are therefore usualiy "dry." The drywell frequency monitoring schedule calls for 105 drywel1s weekly, 91 biweekly, 41 monthly, 151 quarterly, and 371 annually.

Laterals are horizontal drywells positioned under single-shell waste storage tanks to detect radionuclides in the soil which could be indicative of tank leakage. These drywells are monitored by radiation detection probes. Laterals are 4-in. inside diameter steel pipes located 8 to $10 \mathrm{ft}$ below the tank's concrete base. There are three laterals per tank. Laterals are located only in $A$ and $S X$ farms.

The surface level measurements in all waste storage tanks are monitored by manual or automatic conductivity probes, and recorded and transmitted or inputted to the Computer Automated Surveillance System (CASS).

An automatic waste surface level measurement device is manufactured by the Food Instrument Company (FIC). The instrument consists of a conductivity electrode (plummet) connected to a calibrated steel tape, a steel tape reel housing and a controller that automatically raises and lowers the plummet to obtain a waste surface level reading. The controller can provide a digital display of the data and also transmit the reading to the CASS. Some tanks have gauges connected to CASS and others are read manualiy. 
Annulus

Liquid Observation Well (LOW)

Thermocouple (TC)

In-tank

Photography
The annulus is the space between the inner and outer shells on DSTs. Drain channels in the insulating and/or supporting concrete carry any leakage to the annulus space where conductivity probes are installed. Alarms from the annunciators are recoived by CASS. Continuous Air Monitoring (CAM) alarms are also located in the annulus. The annulus conductivity probes and radiation detectors are the primary means of leak detection for all DSTs.

In-tank 1iquid observation wells are used for monitoring the interstitial liquid level (ILL) in single-shell waste storage tanks. The wells are constructed of fiberglass or TEFZEL*-reinforced epoxy-polyester resin, sized to extend to within 1 in. of the bottom of the tank steel liner. They are sealed at their bottom ends and have a nominal outside diameter of $3.5 \mathrm{in}$. Three probes are used to monitor changes in the ILL: acoustic; gamma; and neutron, which can indicate intrusions or leakage by increases or decreases in the ILL. There are 58 LOWs (56 are in operation) installed in SSTs that contain or are capable of containing greater than 50,000 gal of drainable interstitial liquid, and in two DSTs only. The LOWs installed in two DSTs, (102-SY and 103-AW Tanks), are constructed of stee1 and are used for special surveillance purposes only.

A thermocouple is a thermoelectric device used to measure temperature. More than one thermocouple on a device (probe) is called a thermocouple tree. In DSTs there may be one or more thermocouple trees in risers in the primary tank. In addition, in DSTs only, there are thermocouple elements installed in the insulating concrete, the lower primary tank knuckle, the secondary tank concrete foundation, and in the outer structural concrete. These monitor temperature gradients with in the concrete walls, bottom of the tank, and the domes. In SSTs, there may be one or more thermocouple trees installed directly in a tank, although some SSTs do not have any trees installed. A single thermocouple may be installed in a riser, or lowered down an existing riser or LOW. There are also four thermocouple laterals beneath Tank 105-A in which temperature readings are taken in 34 thermocouples.

In-tank photographs may be taken to aid in resolving in-tank measurement anomalies and determine tank integrity. Photographs help determine sludge and liquid levels by visual examination.

* TEFzEL, a trademark of E. I. du Pont de Nemours \& Company 
4. INYENTORY AND STATUS BY TANK - COLUMN CALCULATIONS (SINGLE-SHELL TANKS) COLUMU HEADING

Total Waste Solids Volume plus Supernatant liquid.

Supernatant Drainable Llquid Remaining minus Drainable Interstitial. Liquitd Supernate is usually derived by subtracting the solids level measurement from the liquid level measurement.

Drainable

Interstitial

Total Jet

Pumped

Drainable

Liquid

Remaining

Pumpable

Liquid

Remaining

Sludge

Saltcake

Solids Volume

Update

Solids Update Source - See Footnote

Last Photo

Date

Change Since Last Monthly Report
Drainable Liquid Remaining minus Supernate.

Drainable Interstitial Liquid is calculated based on the saltcake and sludge volumes, using average porosity values or actual data for each tank, when available.

Cumulative total pumped 1979 to date.

Supernate plus Drainable Interstitial.

Drainable Liquid Remaining less undrainable heel volume.

Solids formed during sodium hydroxide additions to waste. sludge usually was in the form of suspended solids when the waste was originally received in the tank from the waste generator. In-tank photographs may be used to estimate the volume.

Results from crystalization and precipitation after concentration of liquid waste, usually in an evaporator. If saltcake is layered over sludge, it is only possible to measure total solids volume. In-tank photographs may be used to estimate the saltcake volume.

Indicates the latest update of any change in the solids volume.

Indicates the source or basis of the latest solids volume update.

Date of latest in-tank photographs taken.

Indicates any change made since the previous month. Explanation for the change follows the Inventory and Status by Tank section. 
WHC-EP-0182-62

APPENDIX B

TANK FARM CONFIGURATION, STATUS, AND FACILITY CHARTS 
WHC-EP-0182-62

This page intentionally left blank. 

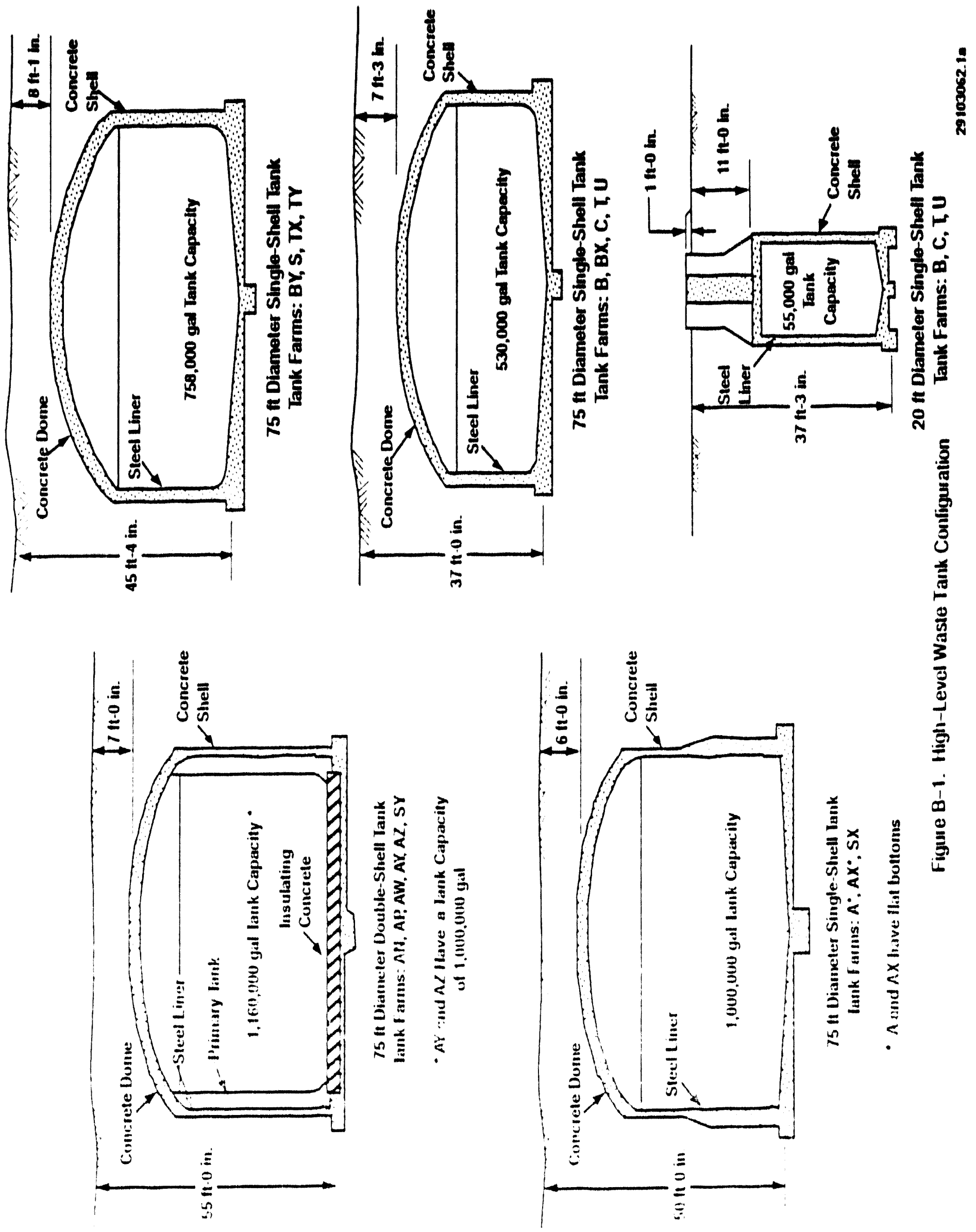


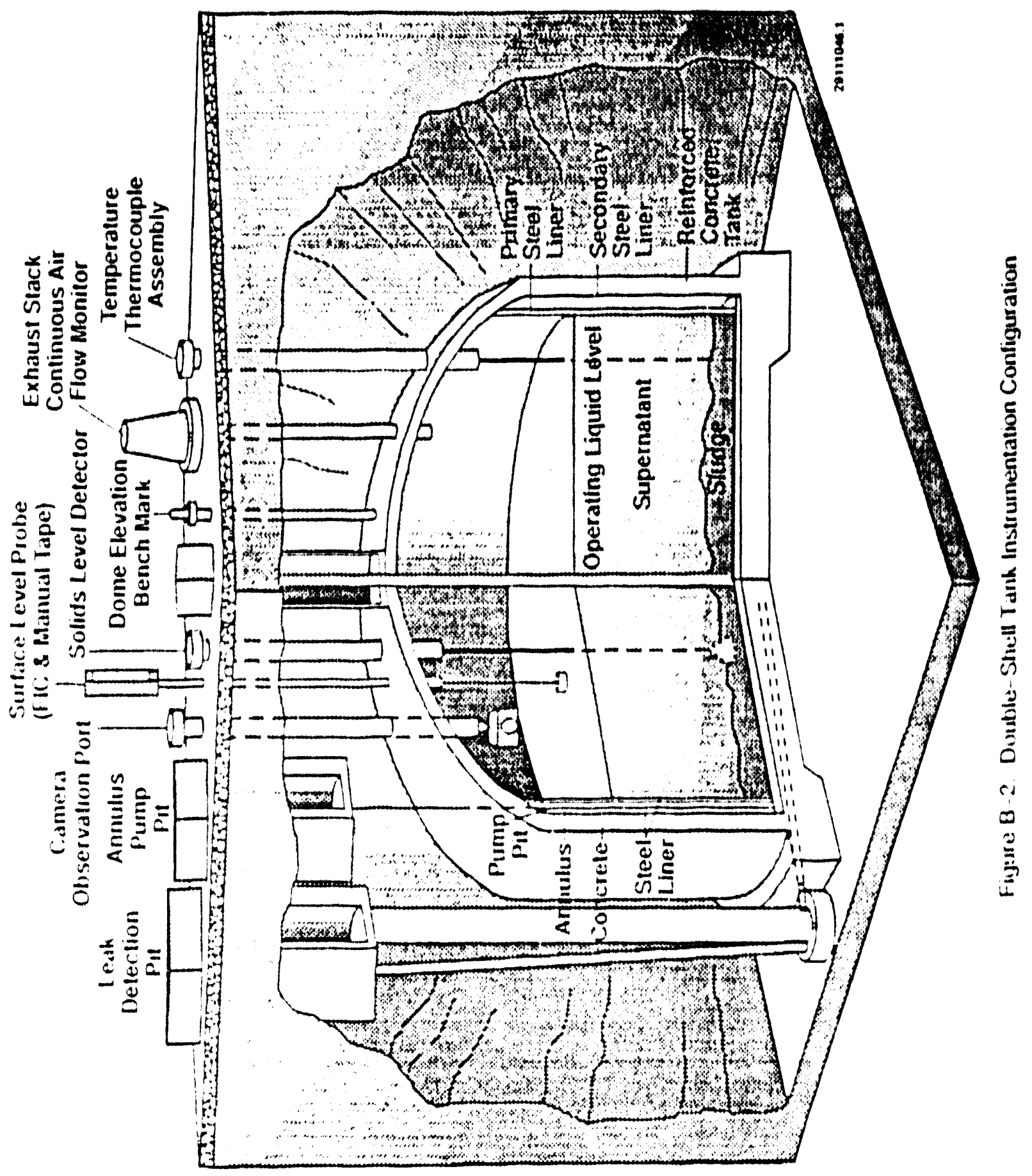




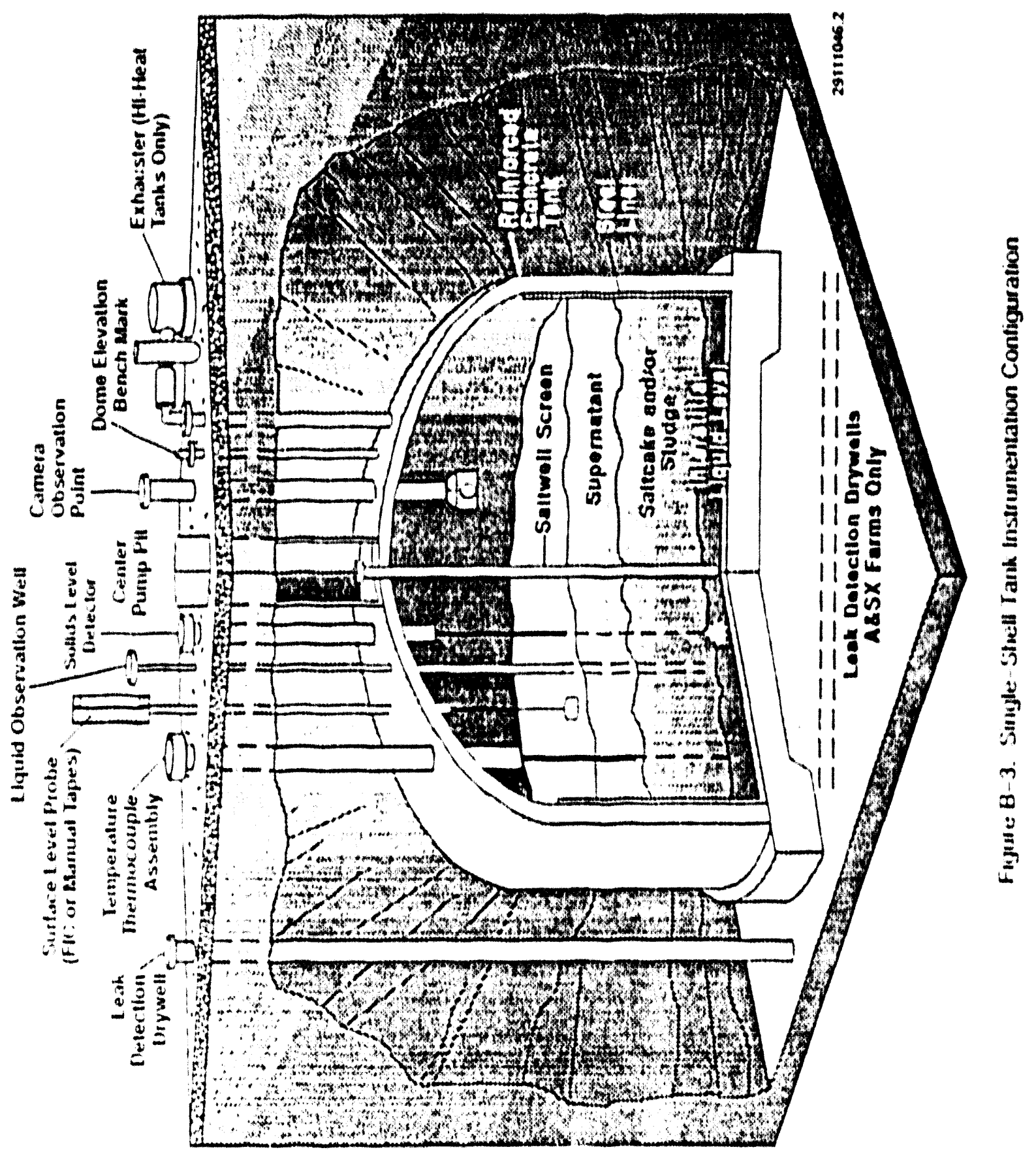


WHC-EP-0182-62

This page intentionally left blank. 


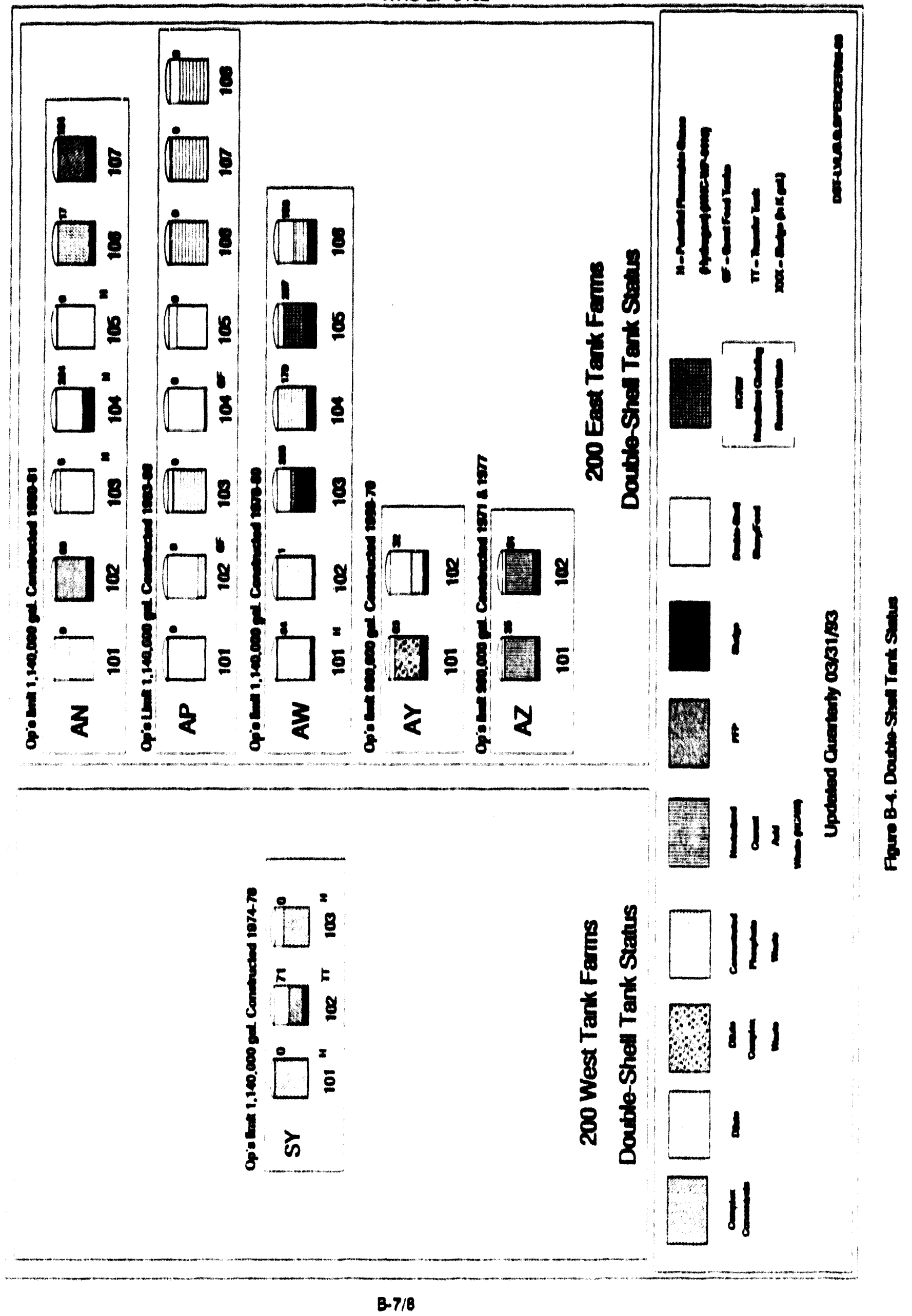


1,000,000 gad. unke Conorratiod 19a4ss

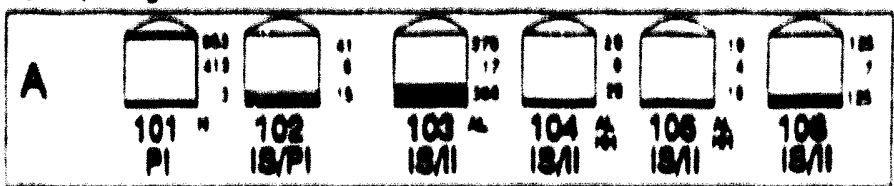

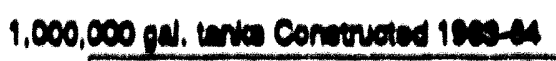

Ax

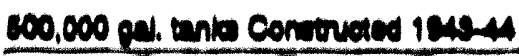

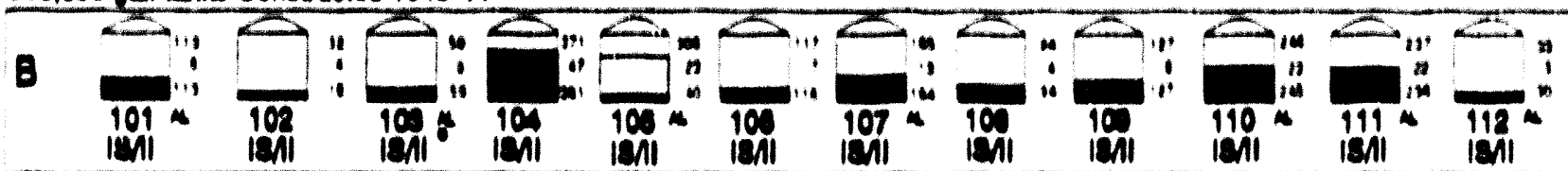

${ }^{201}$ "

500,000 ged. anke Conorveted lespat7

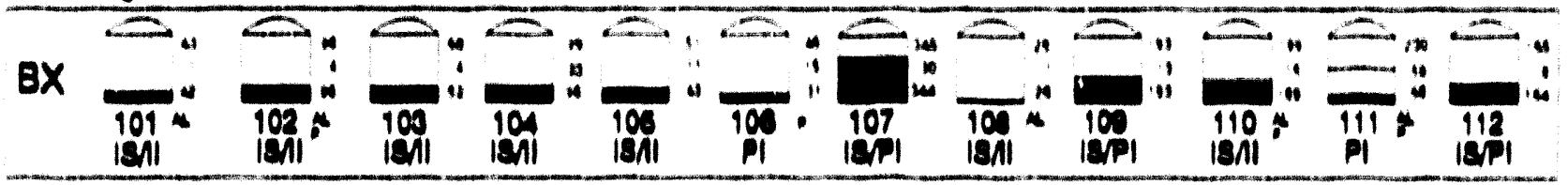

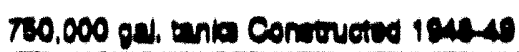

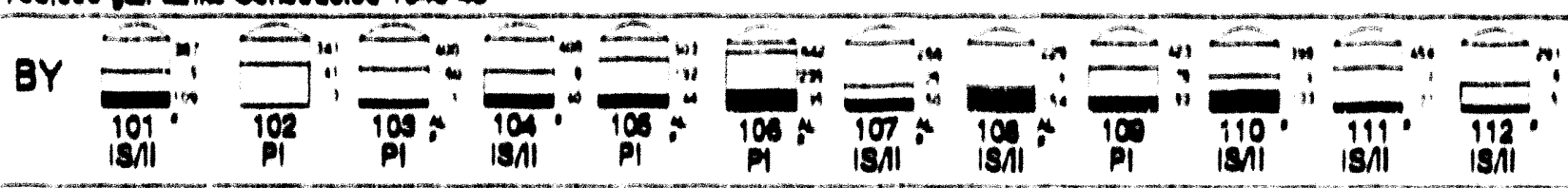

600.000 ped anto Conoruored 18434

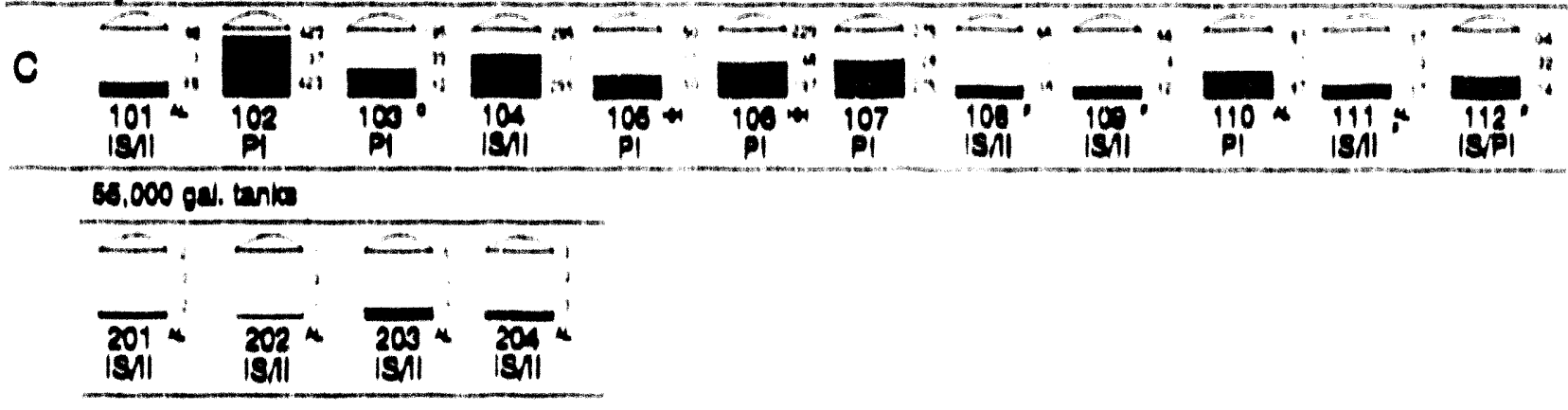

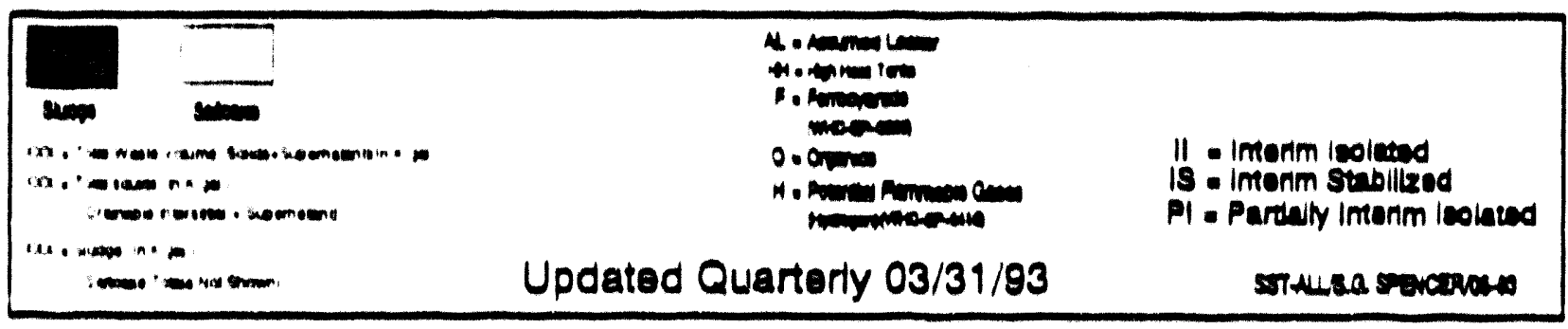

Figure B-5. 200E Single-Shell Tank Status 


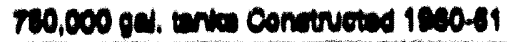

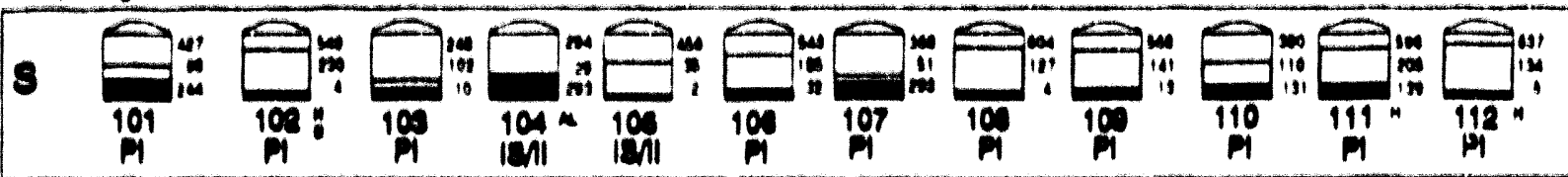

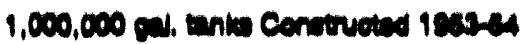

sx

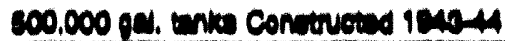

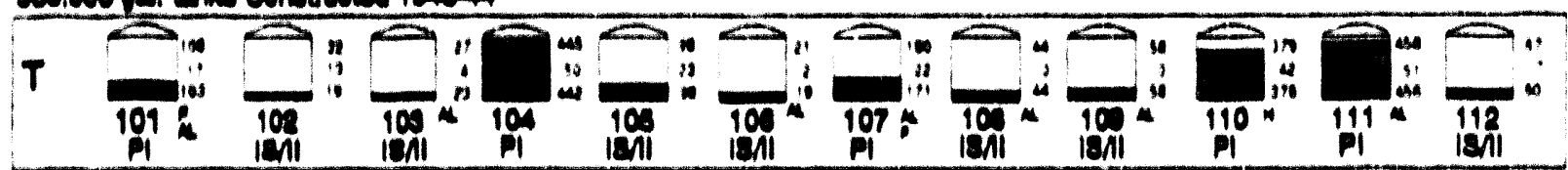

0,000 oul bing

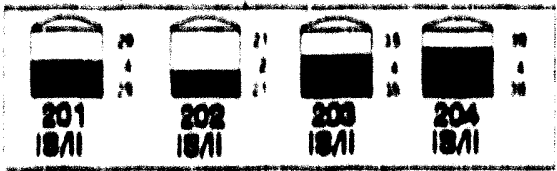

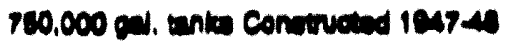

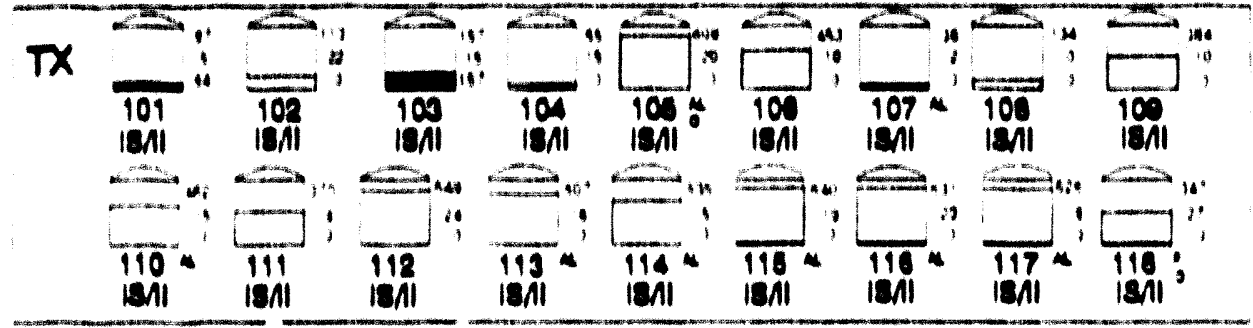

750.000 gel. unia Conenuoted 1931 to

$\mathbf{T}$

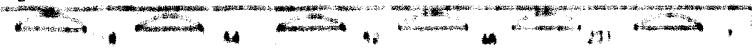

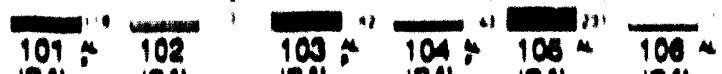

$18 A 1$ 18AI $18 A 1$ ISAI IBAI ISAI

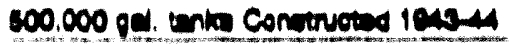

U

-

$=0-10-10$

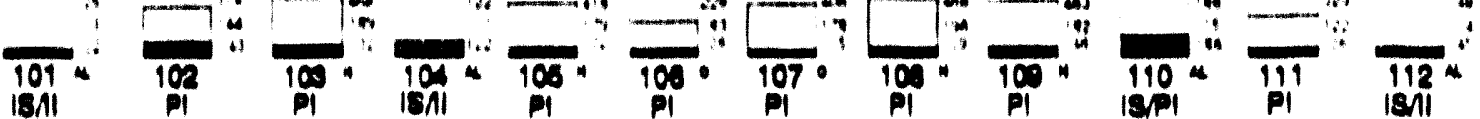

8.000 onl ins

\begin{tabular}{|c|c|c|c|}
\hline 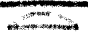 & $x \rightarrow$ & 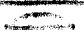 & 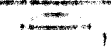 \\
\hline $\begin{array}{l}201 \\
1811\end{array}$ & $\begin{array}{l}202 \\
18 \text { | }\end{array}$ & $\begin{array}{l}800 \\
18 / 1\end{array}$ & $\frac{204}{\mid 8 / 1}$ \\
\hline
\end{tabular}

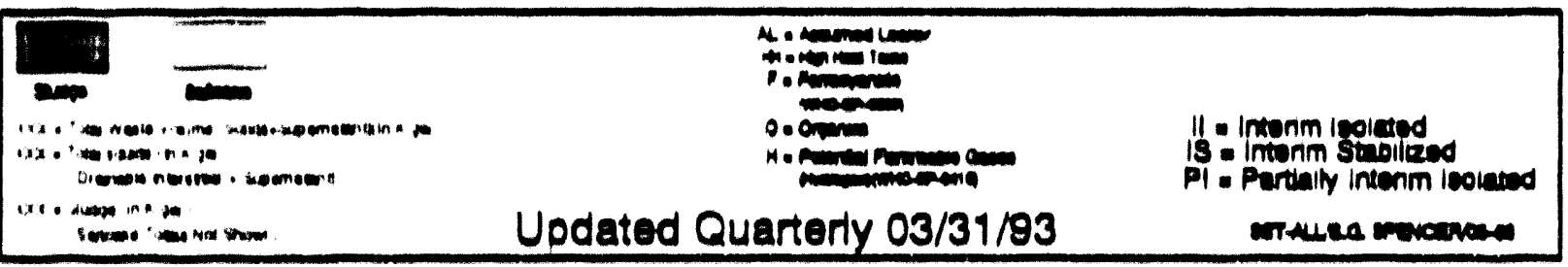

Fig. B-6. 200W Single-Shell Tank Status 


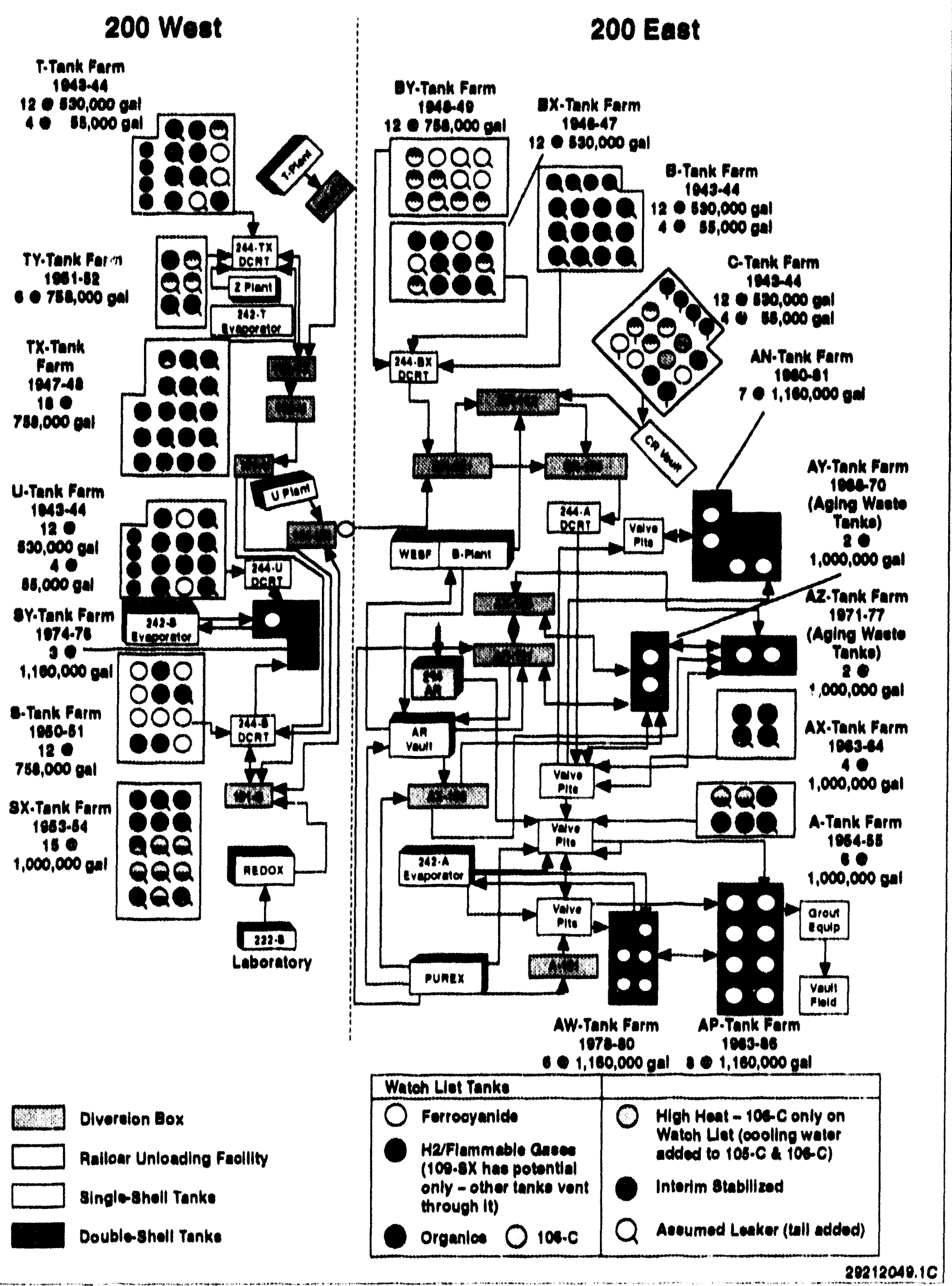




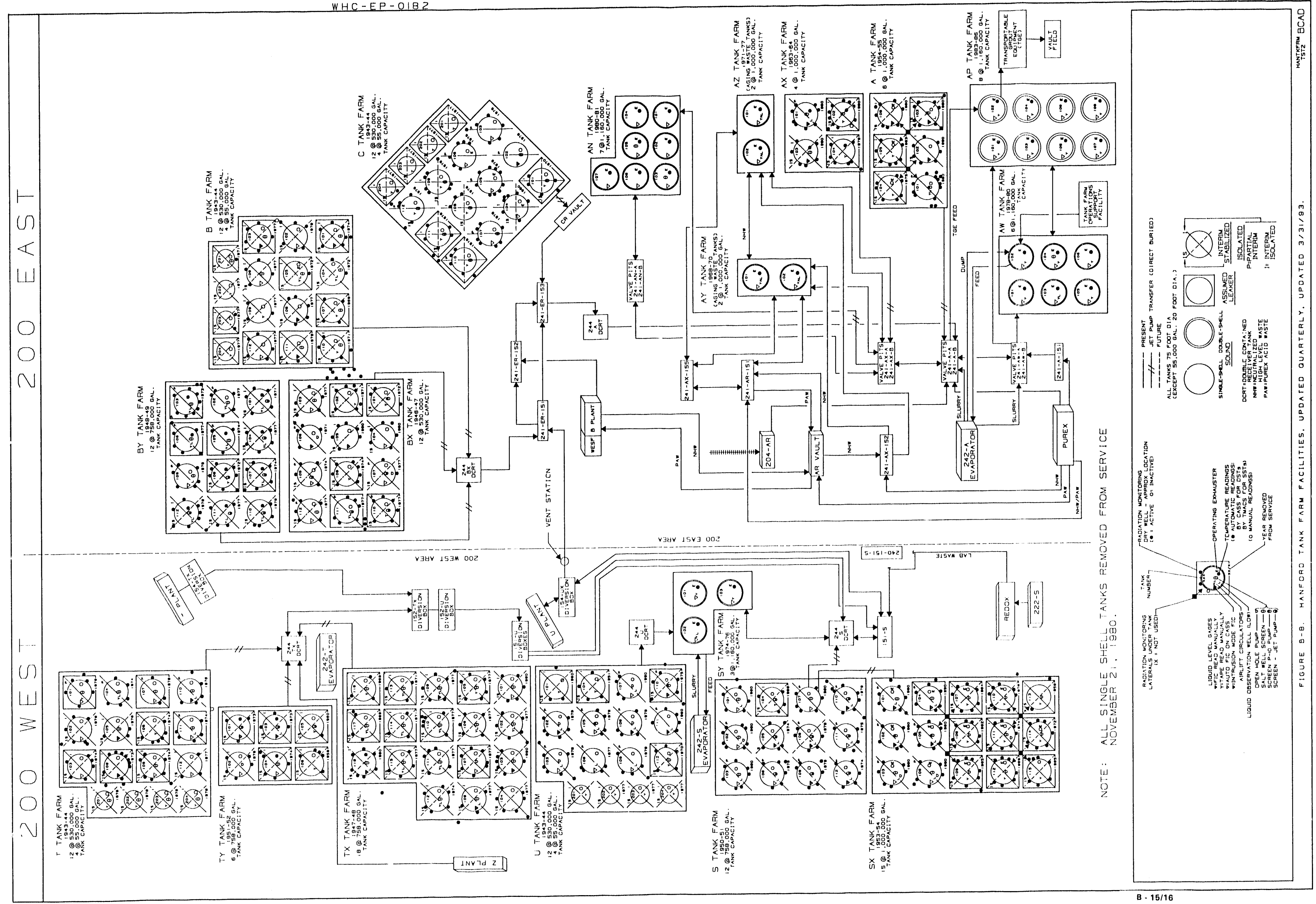


WHC-EP-0182-62

\section{APPENDIX C}

\section{MONTHLY SUMMARY}

TANK USE SUMMARY

INVENTORY SUMMARY BY TANK FARM INVENTORY AND STATUS BY TANK 
VHHC-EP-0182-02

This page intentionally left blank. 


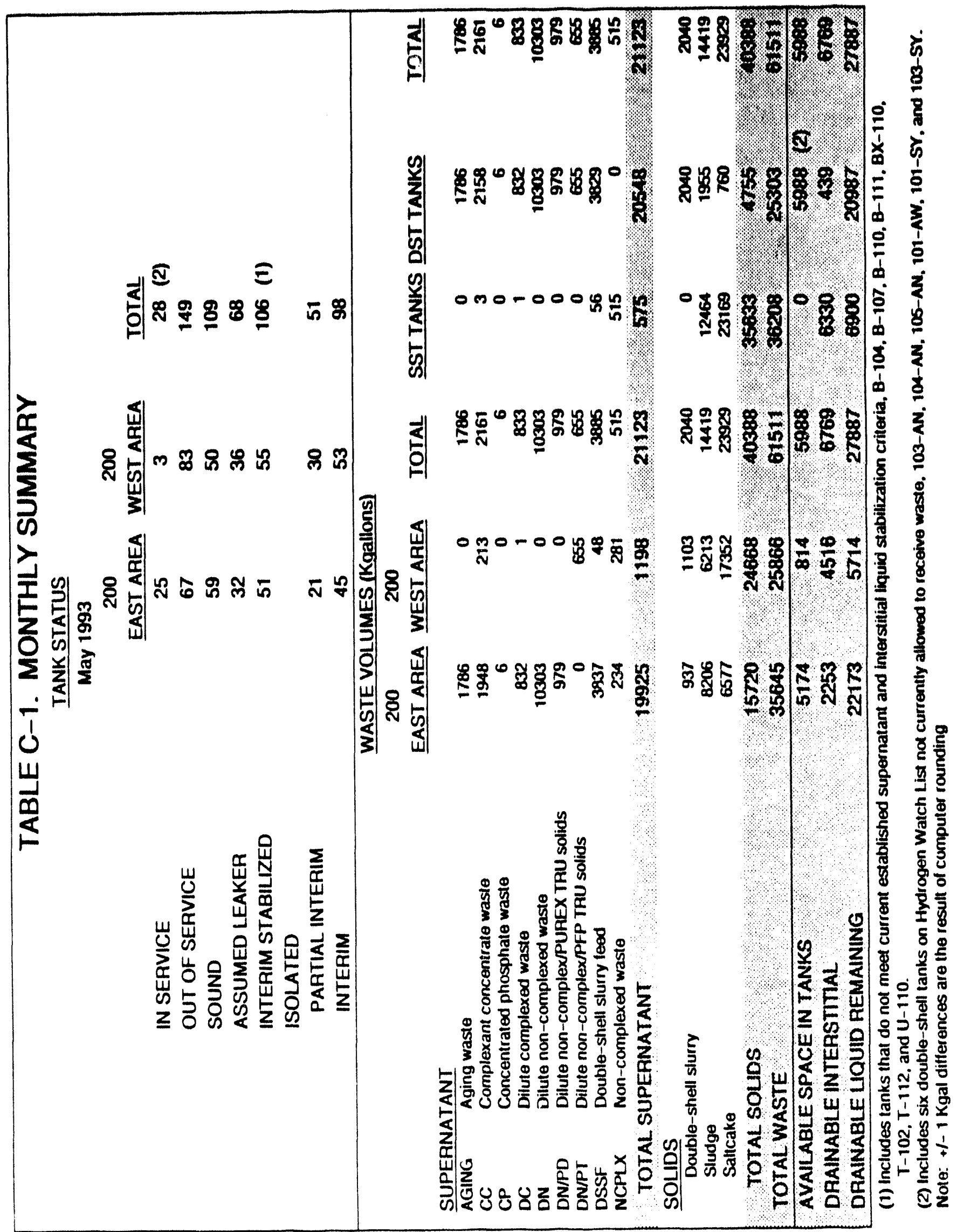




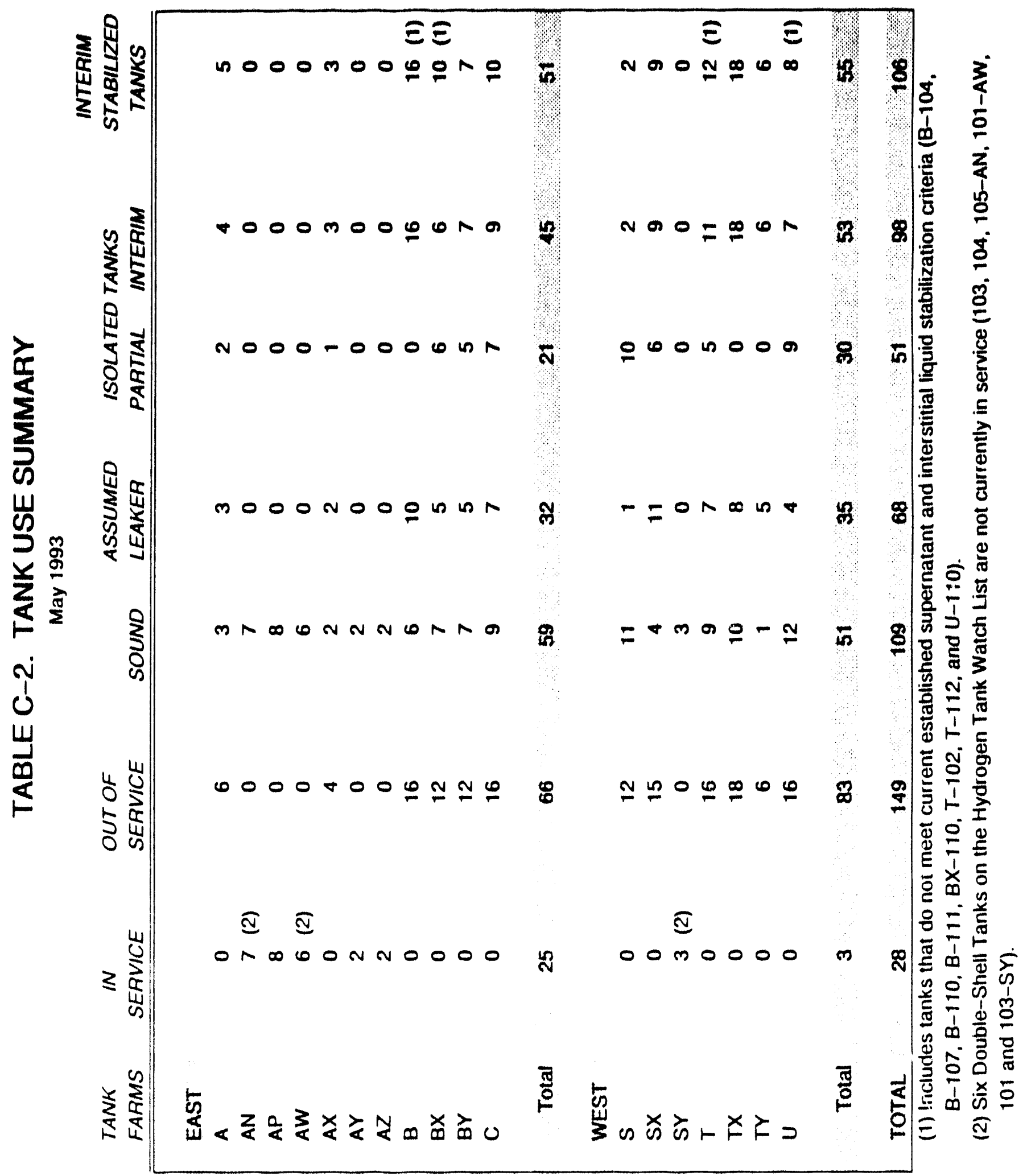


WHC-EP-0182-62

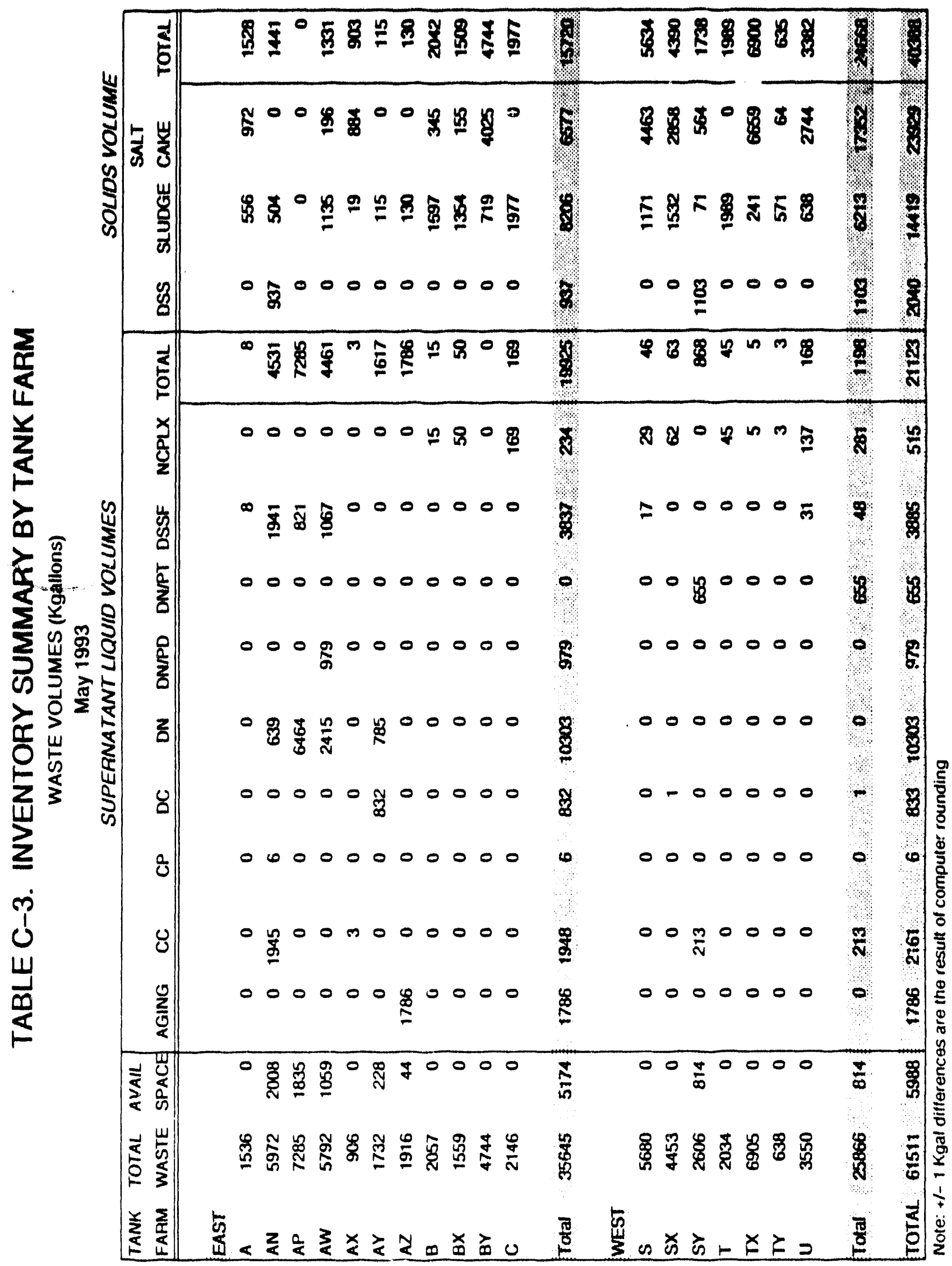


WHC-EP-0182-62

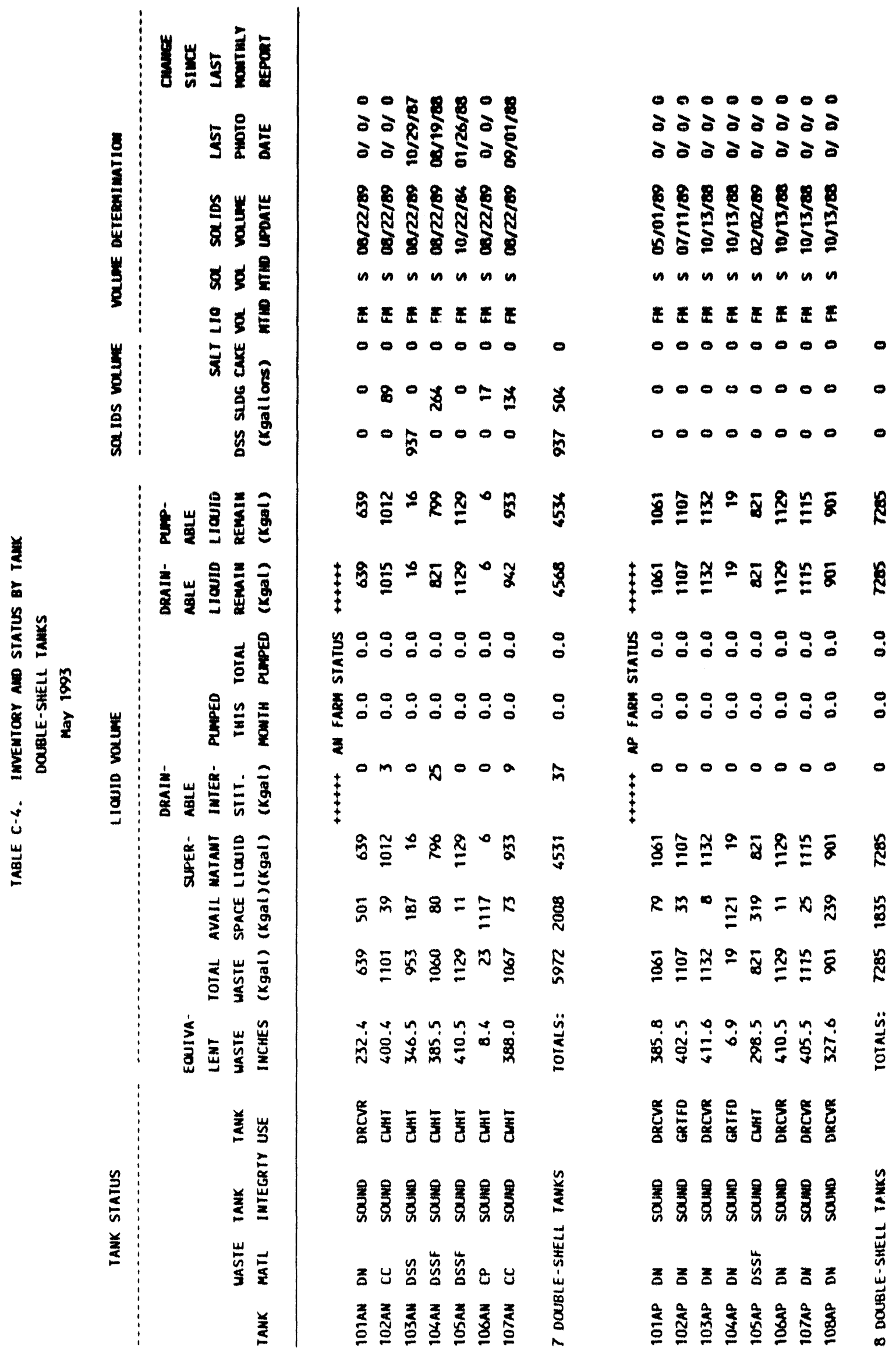


ilHC-E:?-0102-32

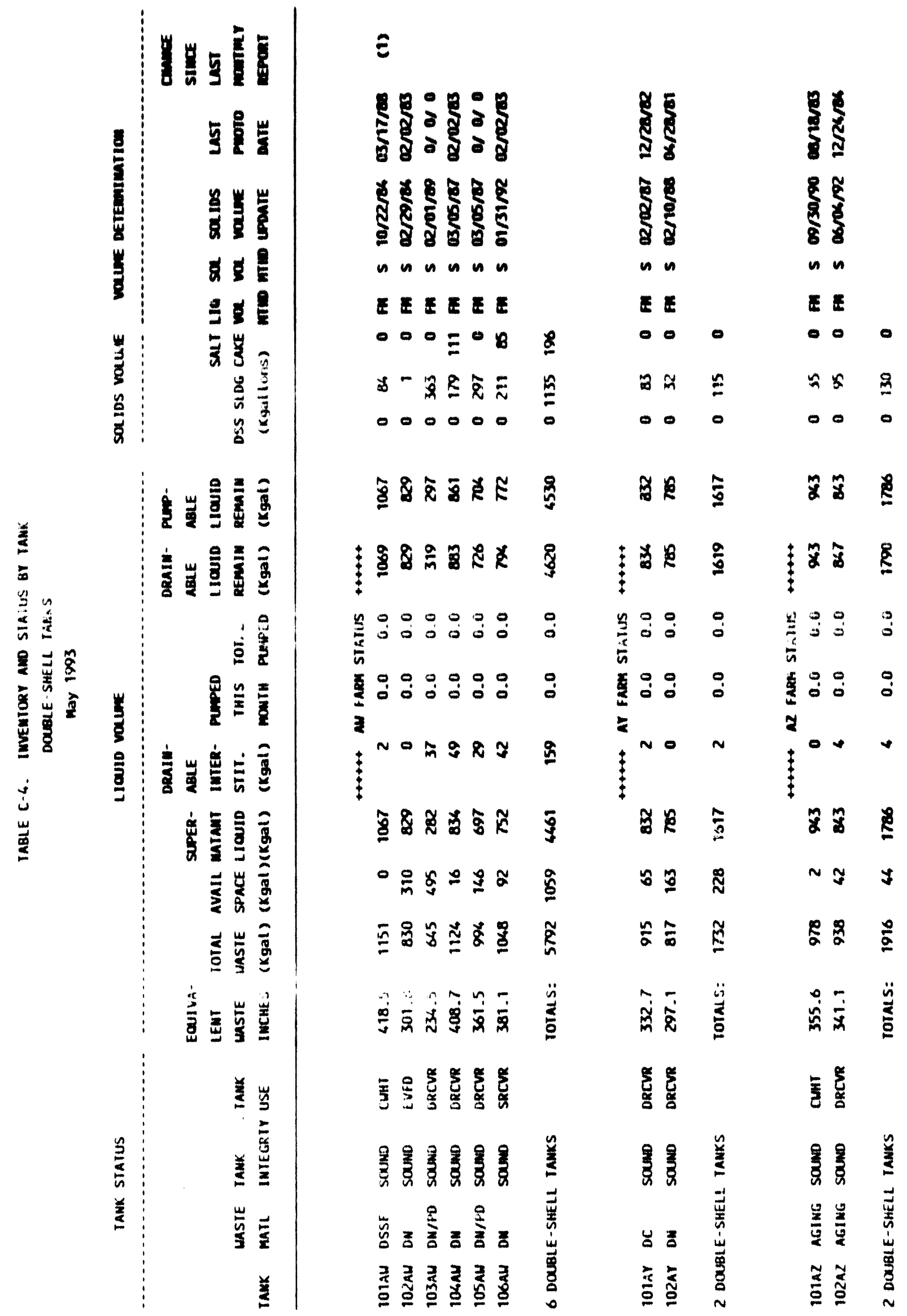




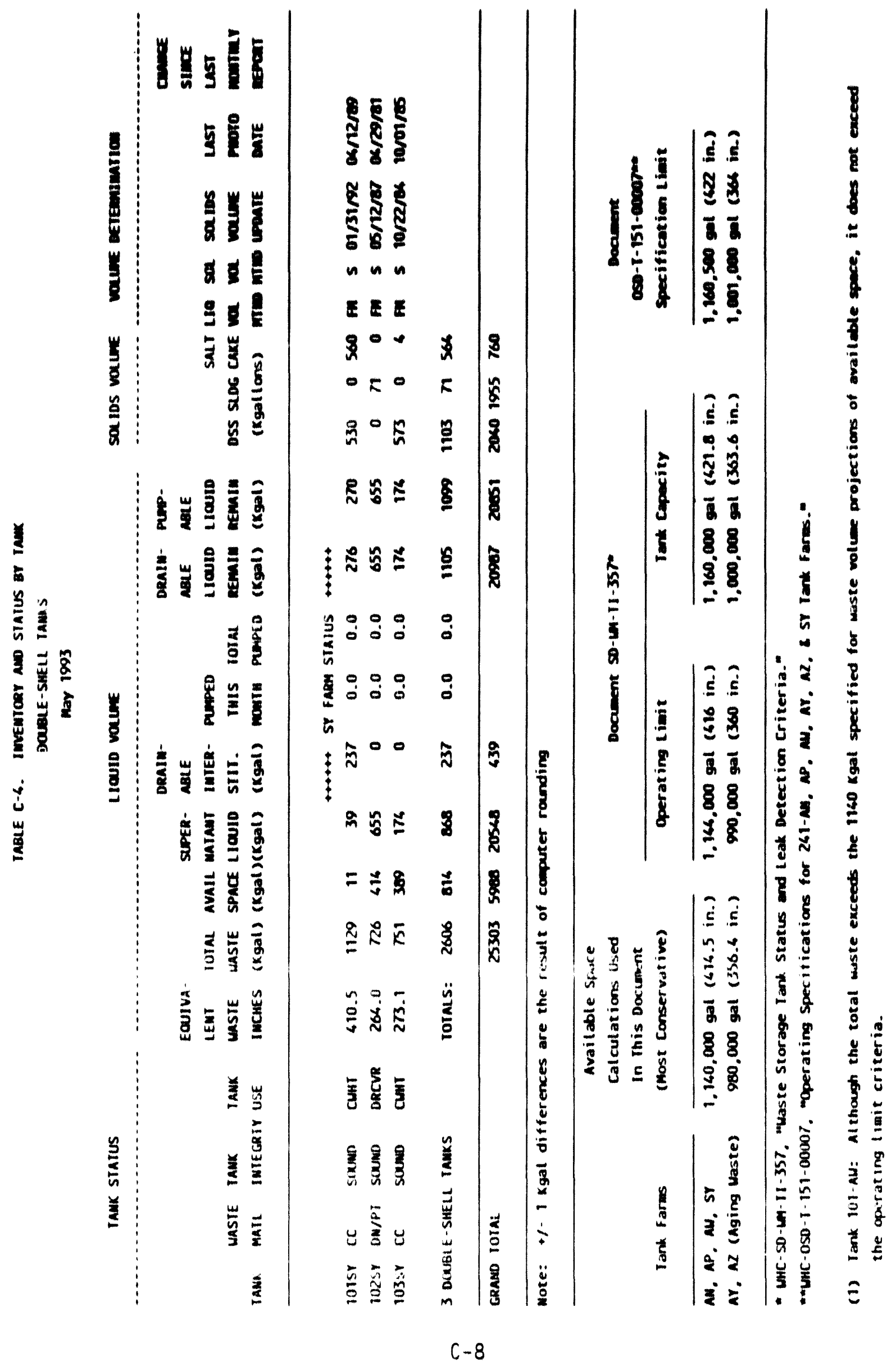




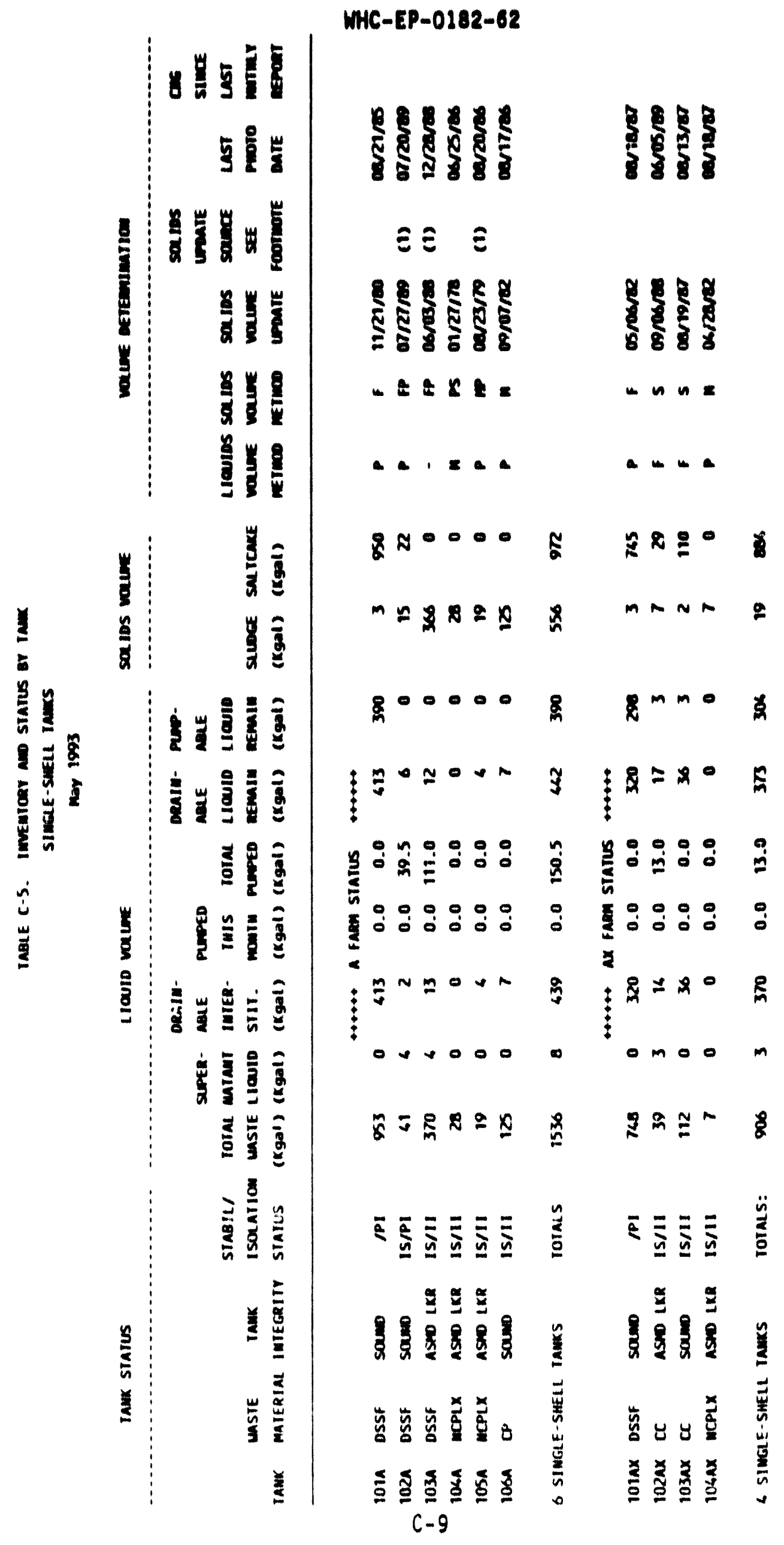




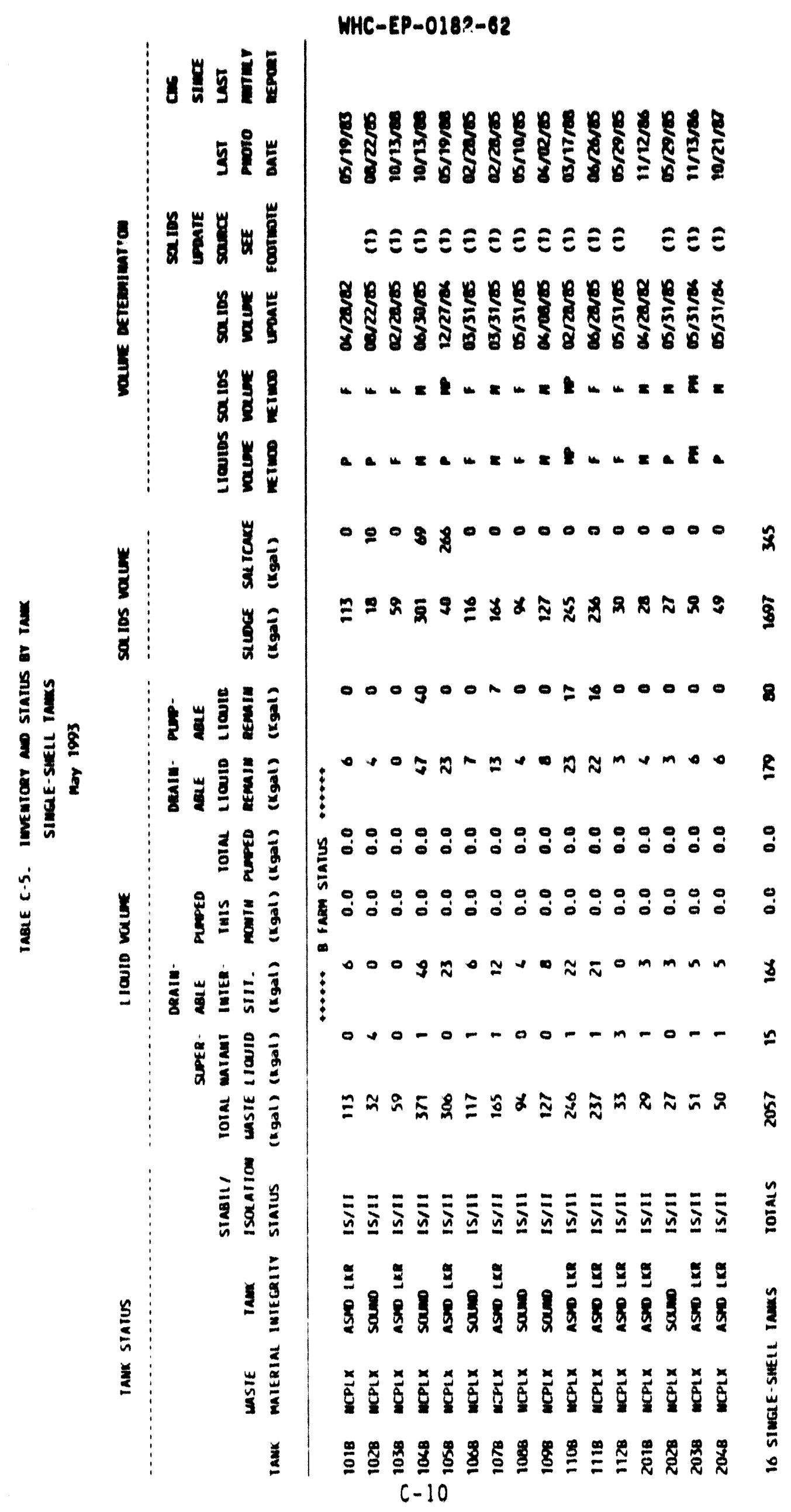




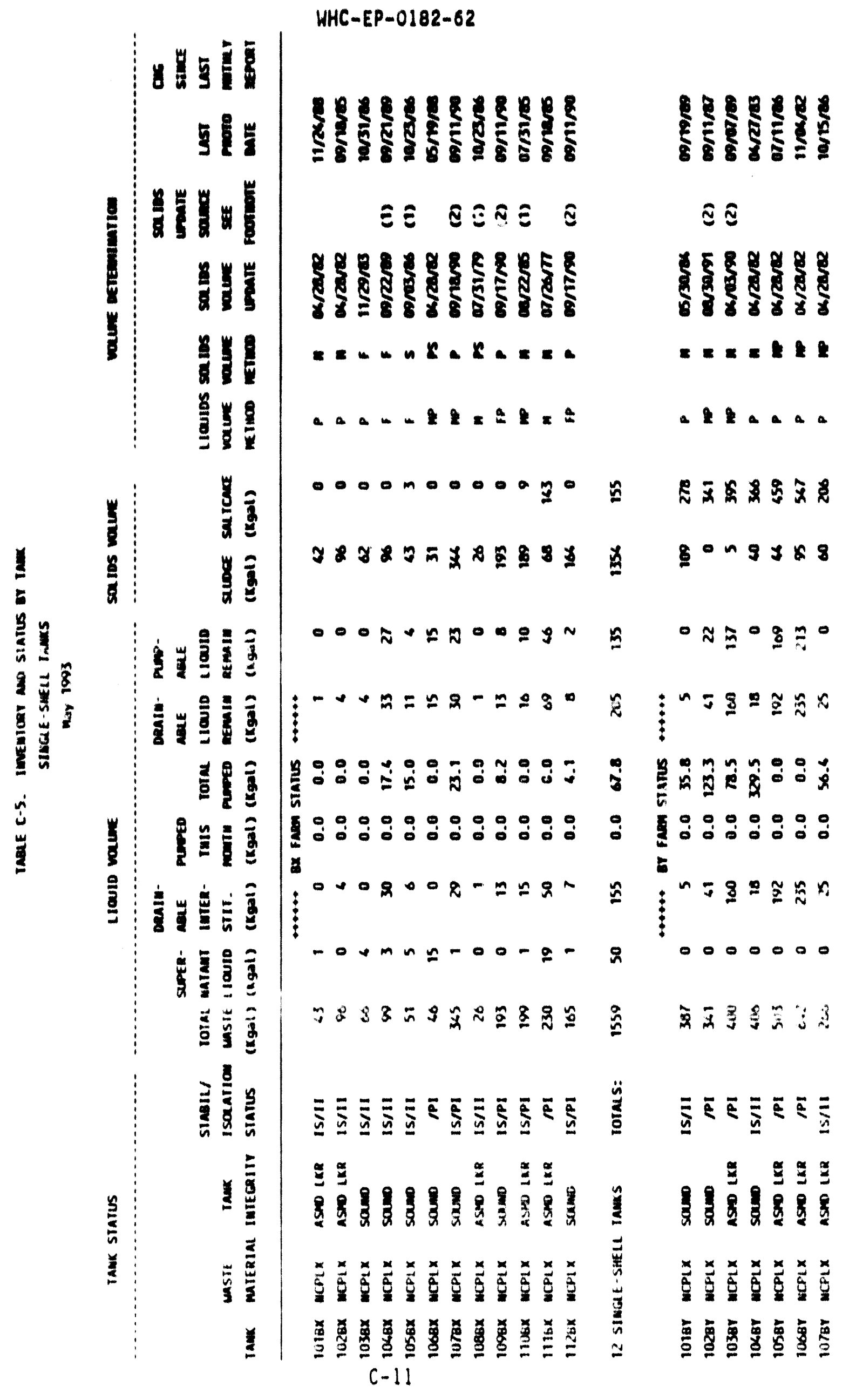




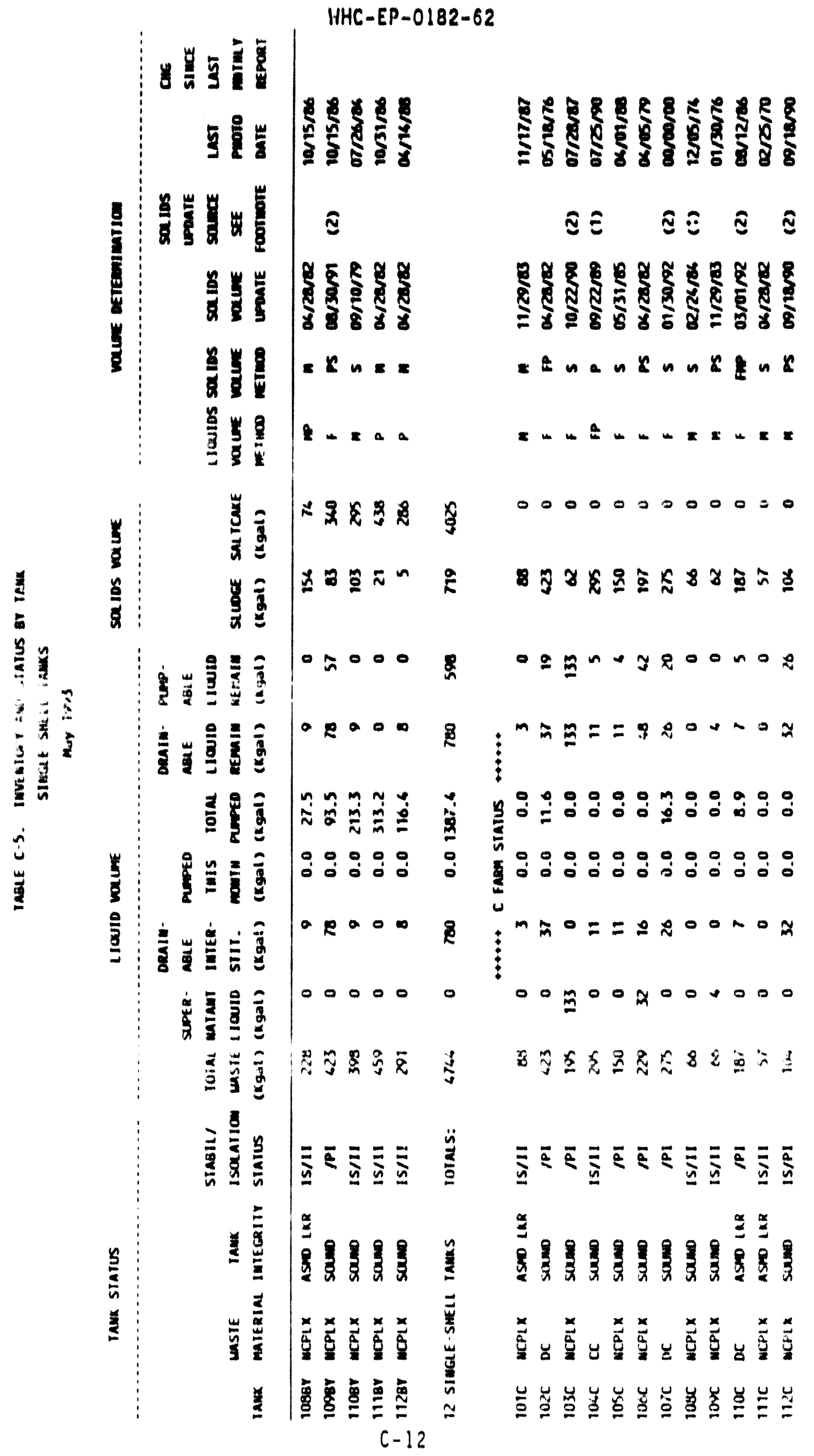




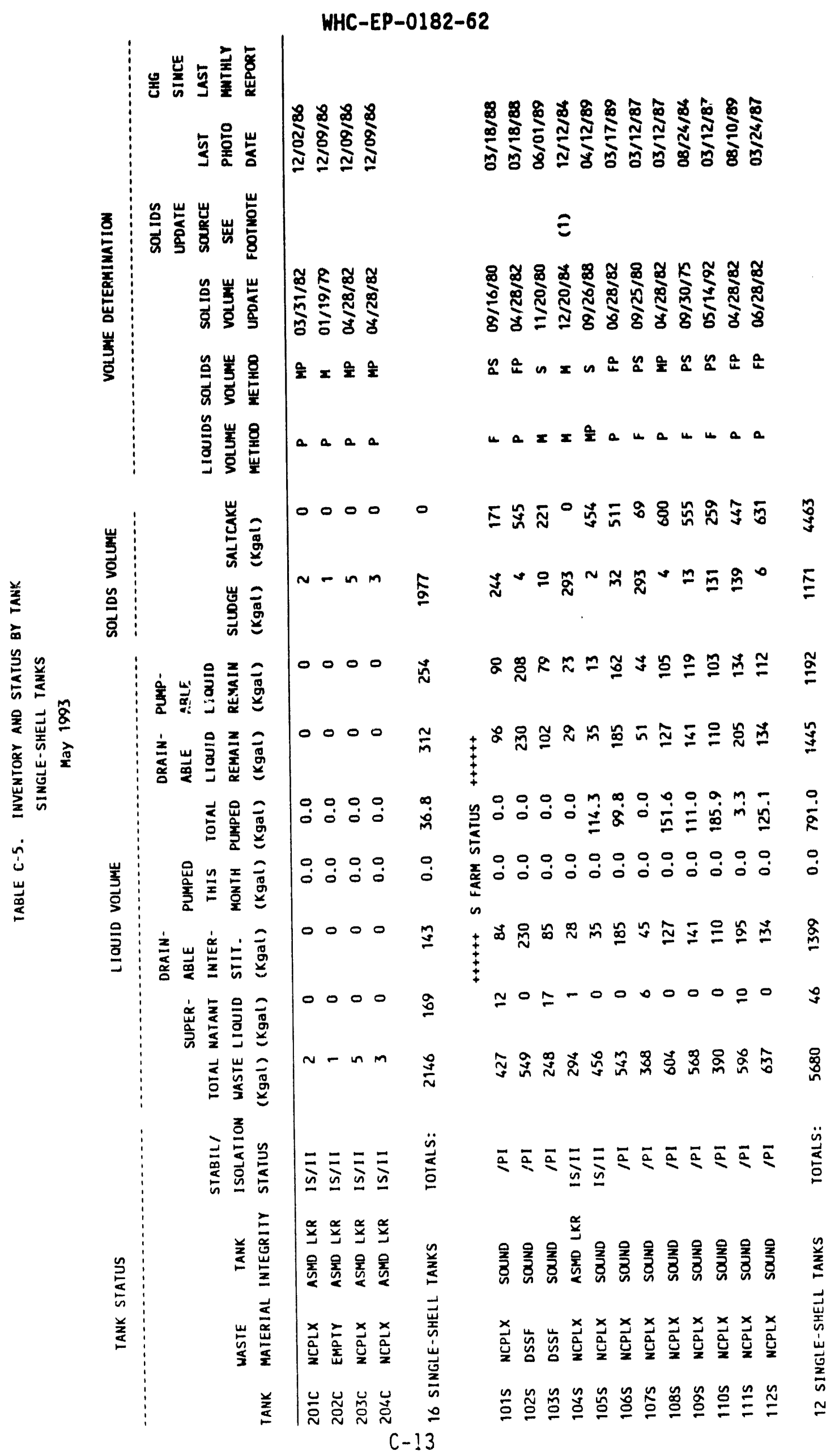




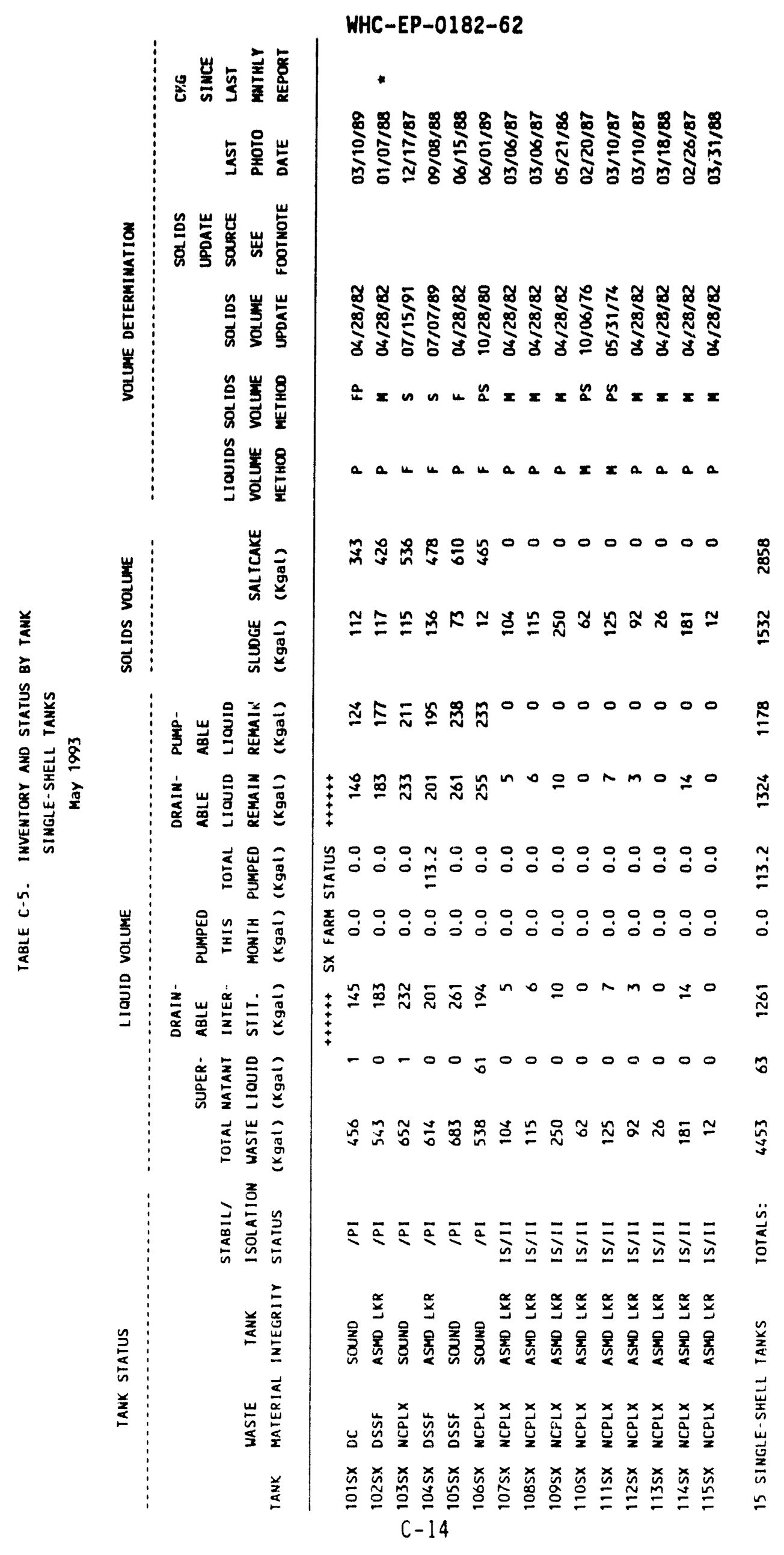




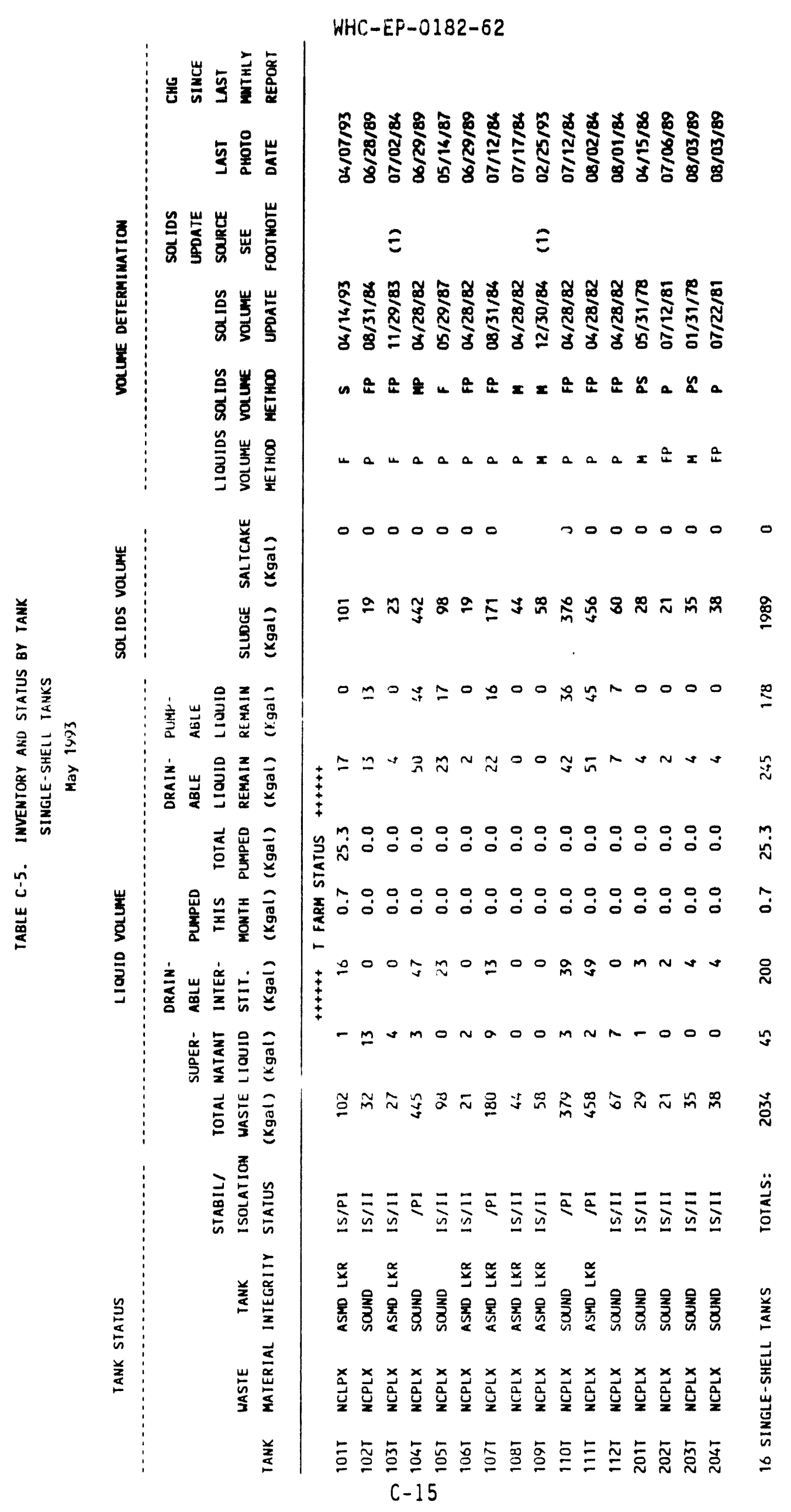




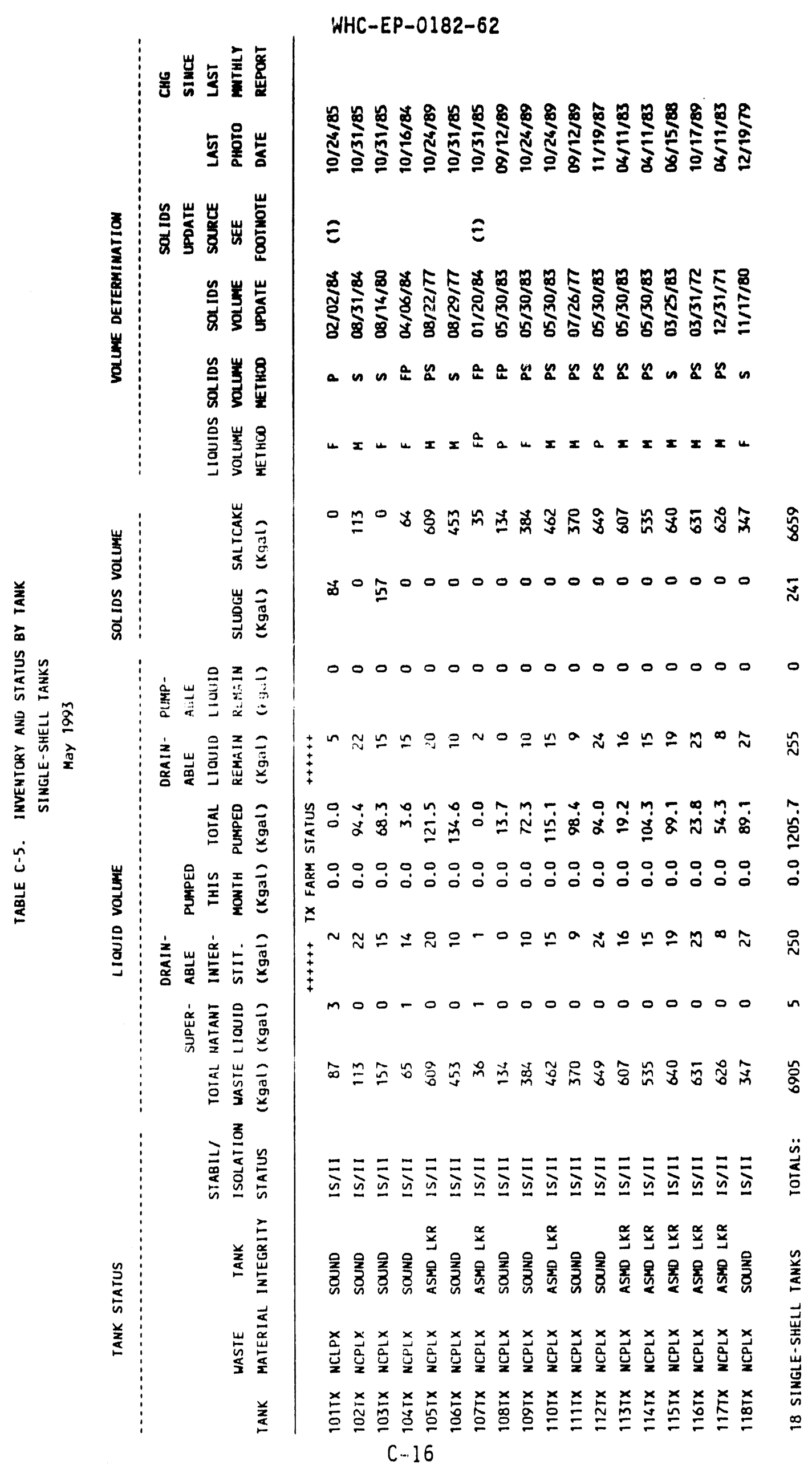




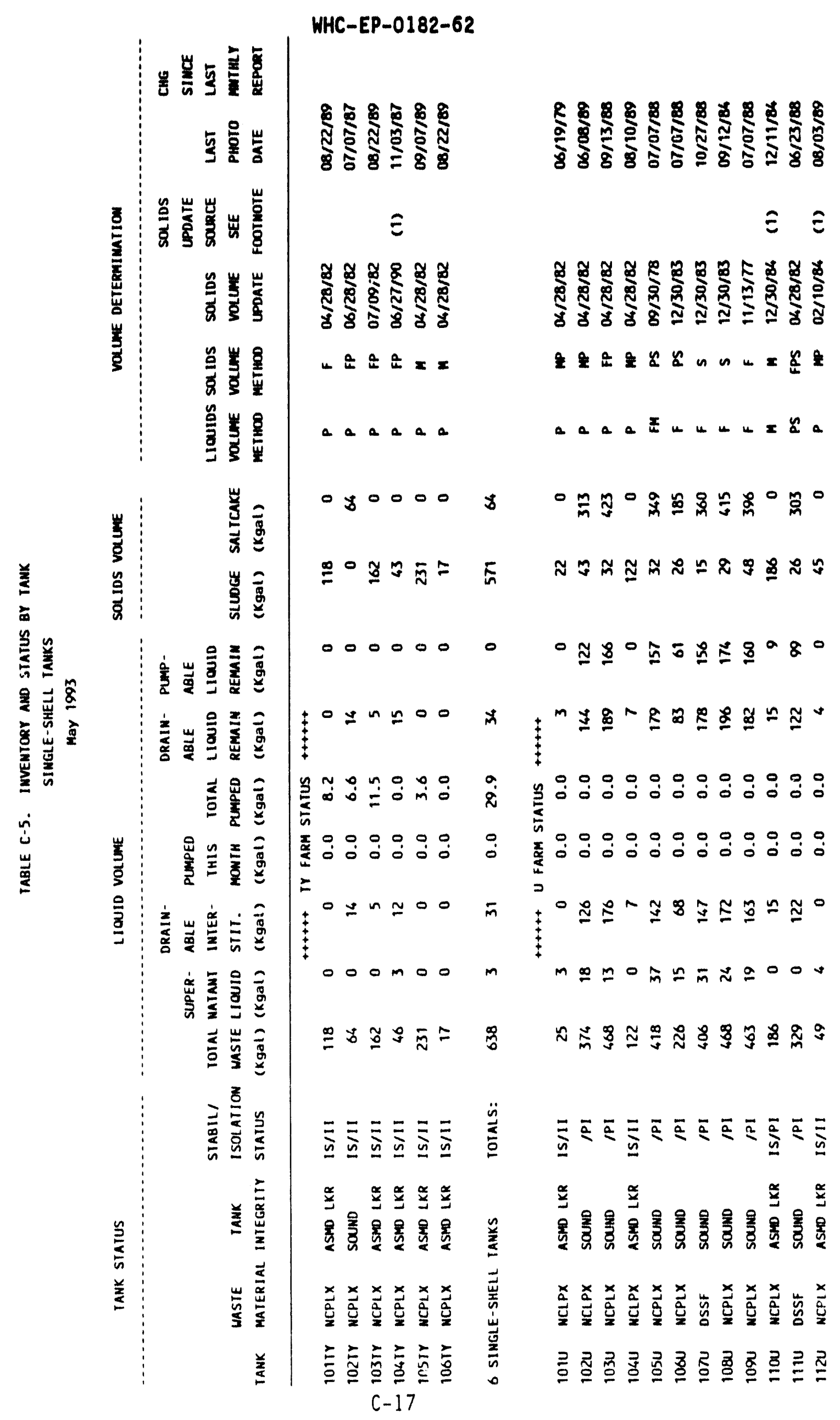




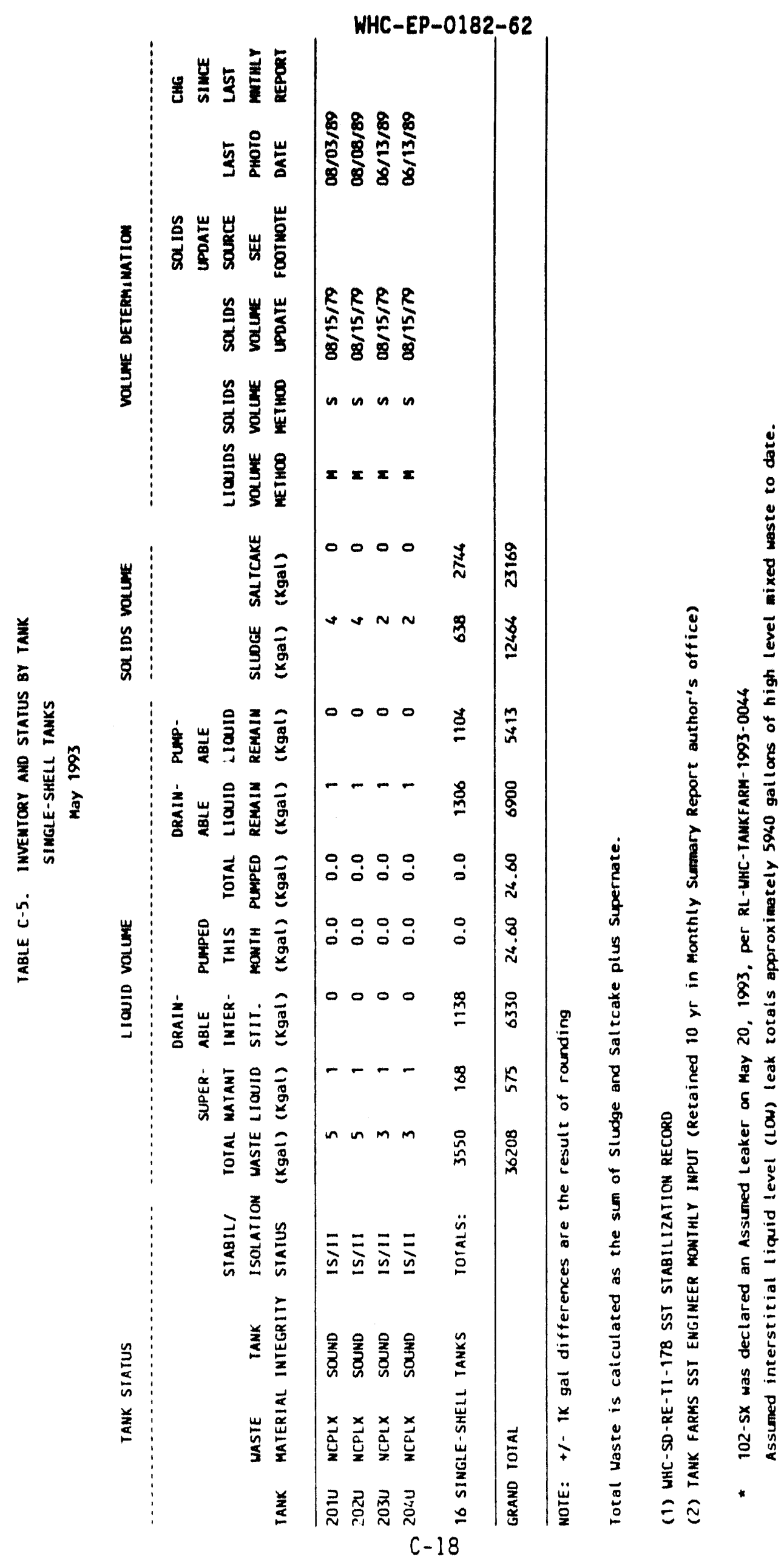


WHC-EP-0182-62

\section{APPENDIX D}

PERFORMANCE SUMMARY 


\section{WHC-EP-0182-62}

This rage intentionally left blank. 


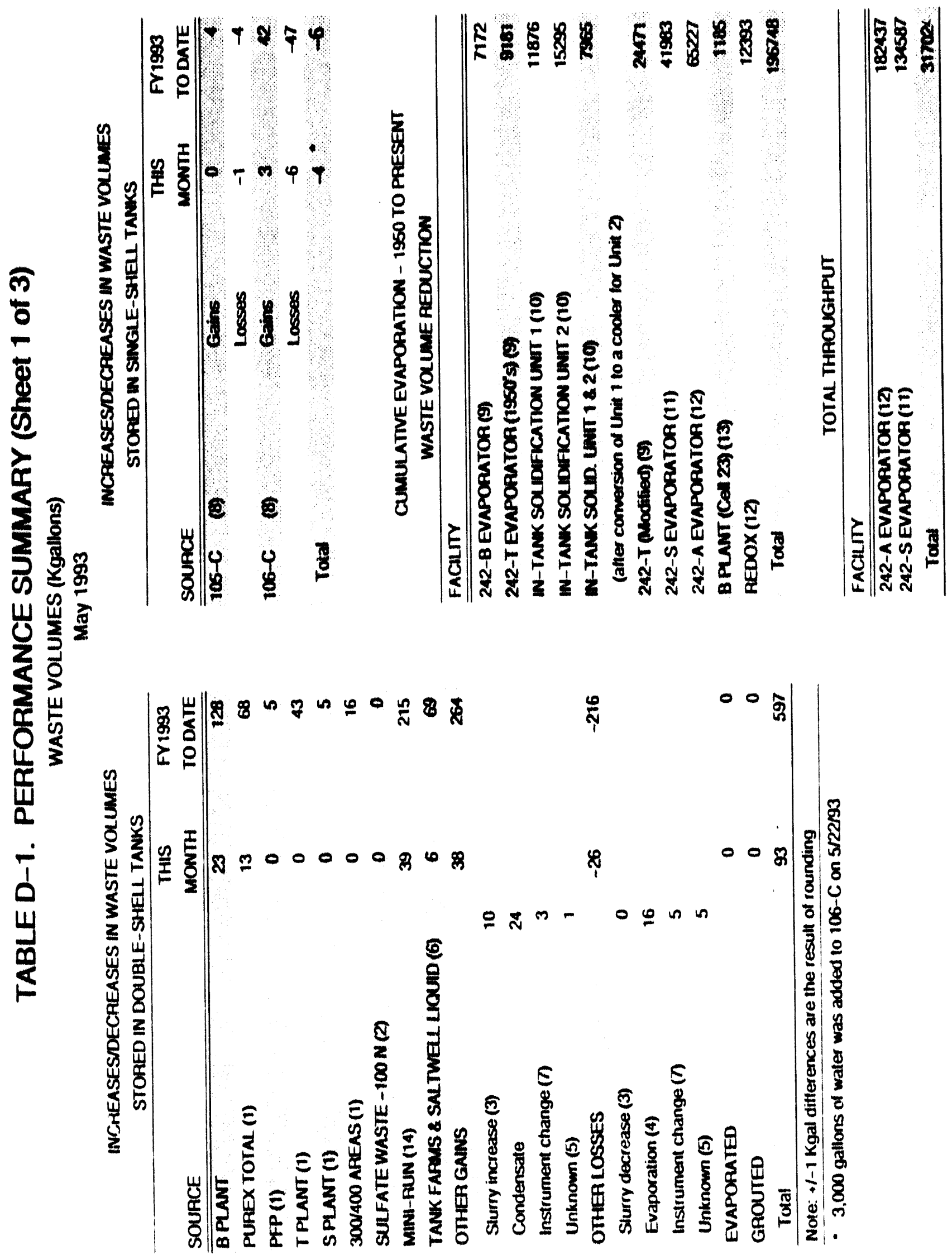

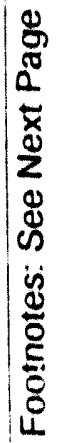




\section{TABLE D-1. Performance Summary (Sheet 2 of 3)}

Footnotes:

INCREASES/DECREASES IN WASTE VOLUMES

(1) Including Flush

(2) Sulfate waste is generated from ton exchange backflushing and sand fllter clean out, resulting in sulfate waste $\left(\mathrm{Na}_{2} \mathrm{SO}_{4}\right)$.

(3) Slurry increase/growth is caused by gas generation within the waste. The gas which is trapped in the waste expands in the tank causing the surface level and volume to increase. Slurry decrease results from the pertodic release of gas in the waste.

(4) Aging waste tanks

(5) Unknown waste gains or losses may be the result of rounding calculations, clean water slowly leaking through a valve, changes in levels (expansion/contraction) because of anbient temperature changes, different measuring devices being used by Tank Farm operators, transfers taking place during the end of the month, Tank Farm activities such as miscellaneous water additions not associated with facllity waste generation, or the addition of water which is added to aging waste tanks and then evaporated off.

(6) Includes Tank Farms miscellaneous flushes (flushes are used to "clean out" pipelines and reduce personnel exposure, reduce potential for waste incompatibility, prevent line plugging, and reduce waste content of potential spills or leaks), and saltwell liquid, which results from pumping of single-shell tanks to double-shell tanks.

(7) Liquid level measurement instrument changes from the automatic FIC to manual tape (and vice versa) result in unusual gains or losses because the manual tape may rest on an uneven crust surface giving a different reading from that of the automatic FIC. These instrument changes are made when the automatic FIC is out of service and the reading from the manual tape is used for reporting purposes. The reported reading reverts back to the automatic FIC when it is repaired.

(8) Water is periodically added to $105-C$ and $106-C$ to provide evaporative cooling. Losses due to evaporation are calculated assuming all losses are evaporative losses. Orywell monitoring for leak detection is done monthly on tank 105-C. Some drywells are monitored weekly and some are monitored every two weeks on tank 106-C. If there are any indications of a leak from these tanks, the assumption that all losses are due to evaporation will be reevaluated. 
WHC-EP-0182-62

TABLE D-1. Performance Summary (Sheet 3 of 3 )

WASTE YOLUME REDUCTION

(9) Currently inoperative. These evaporator systems (242-B and 242-T) were installed in 1952 in each of the two operating areas to remove water from the waste, and ran for approximately 4 yr after which both units were shut down. The 242-T Evaporator was reactivated in December 1965, and shut down again in April 1976.

(10) Currently inoperative. These two in-tank solidification (ITS) units provided in-tank heating to promote in-tank bolling or evaporation. The ITS Unit I started up March 196:., and ITS Unit 2 started up February 1968. In August 1971, ITS Unit I was converted from an evaporator to a cooler for ITS Unit 2. Both units were shut down June 1974.

(11) Currently inoperative. The 242-S Evaporator-Crystallizer was started up November 1973, and shut down March 1980, when tts processing campaign was complated. It is in standby mode with no future mission. This evaporator operates under a vacuum, employing evaporative concentration with subsequent crystalization and precipitation of salt crystals.

(12) Currently inoperattve. The 242-A Evaporator-Crystallizer was started up March 1977, and shut down April 1989 because of regulatory issues, and has remained shut down for subsequent upgrading. The restart schedule has been revised and now specifies mid-July 1993, as the projected start-up date. This evaporator operates under a vacuum, ampioying evaporative concentration with subsequent crystallization and precipitation of salt crystals (forining saltcake).

(13) Currently inoperative. Additional concentration of wastes was completed by using the concentrators at REDOX and $B$ Plant. The REDOX concentrator was us'd from July 1967 to June 1972, while the B Plant concentrator was used from July 1967 to February 1968.

(14) Waste generated for training and testing purposes prior to Evaporator restart 
VHC-EP-0182-62

This page intentionally left blank. 
WHC-EP-0182-62

\section{APPENDIX E}

\section{LIQUID STATUS AND PUMPABLE LIQUID}

REMAINING IN TANKS 
WHC-EP-0182-62

This page intentionally left blank. 


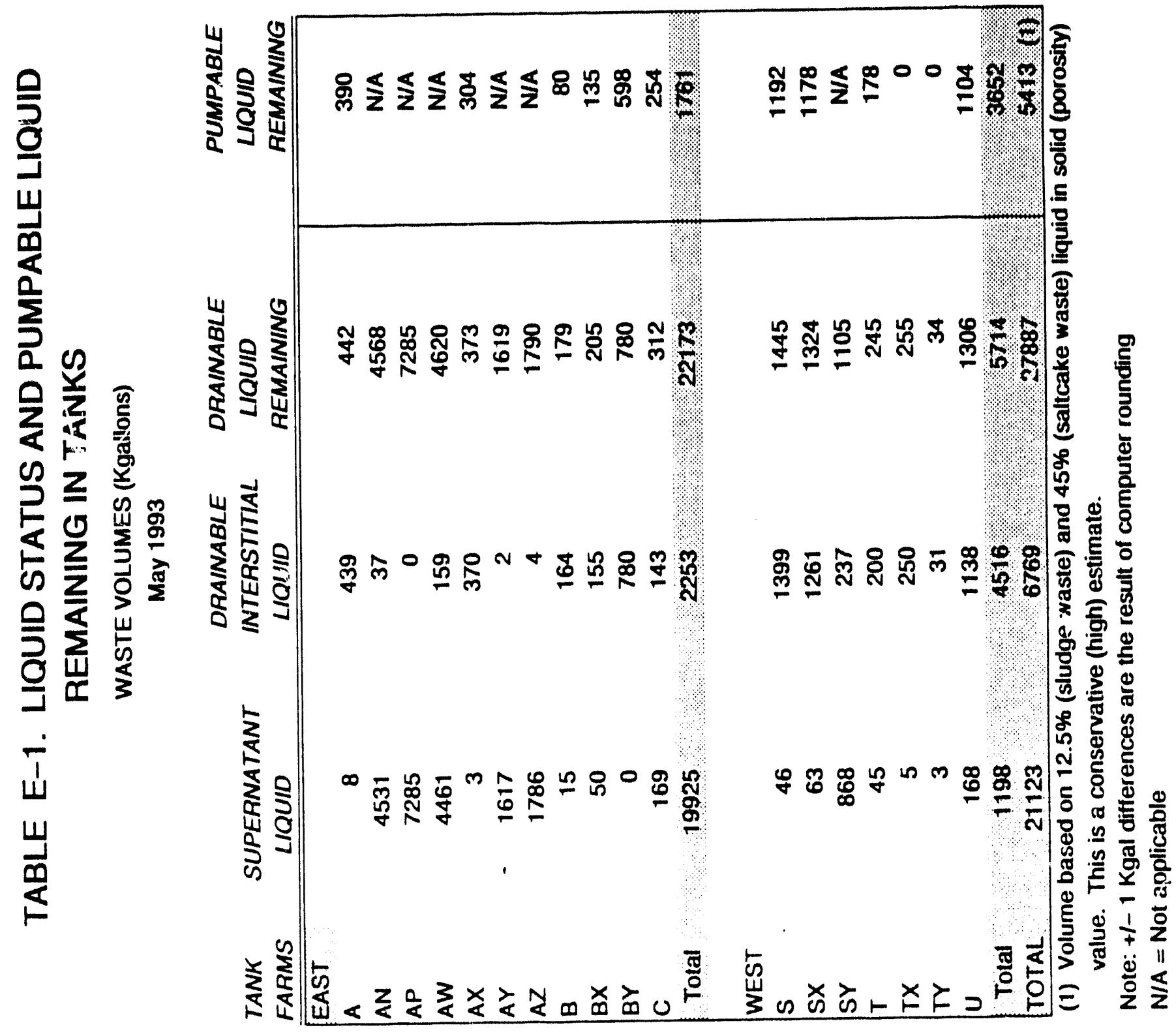


WHC-EP-0182-62

This page intentionally left blank. 
WHC-EP-0182-62

\section{APPENDIX F}

\section{PUMPING RECORD}


WHC-EP-0182-62

This page intentionally left blank.

$F-2$ 


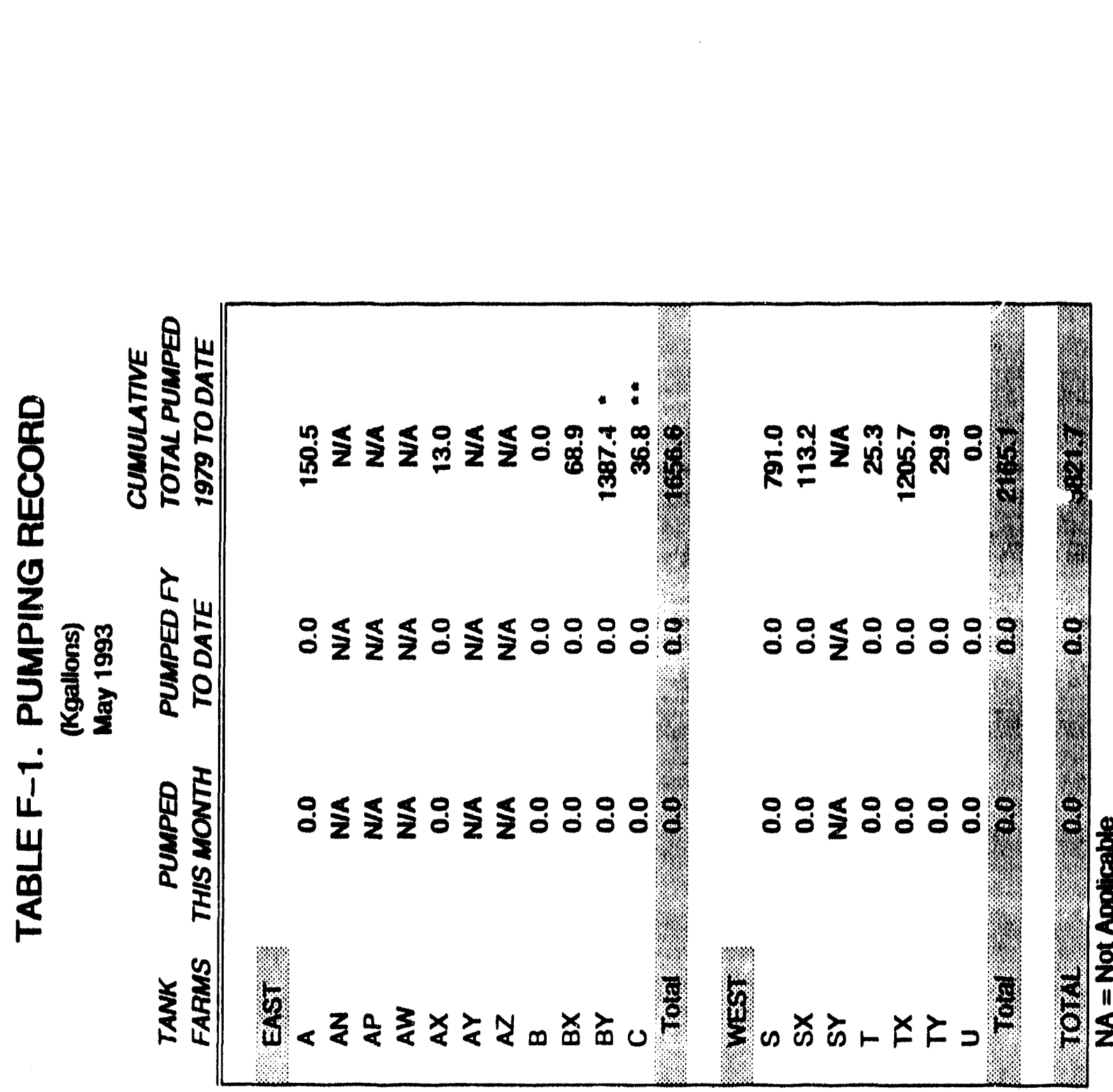

है

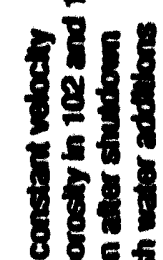

$8 \frac{8}{8}$

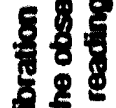

을

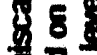

E 8

8

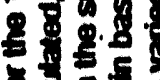

858

$88 \%$

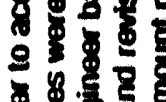

8 है

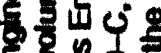

1

들당

ल휴.

क人

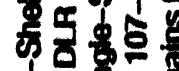
करण 동을 o 85

5 8 용

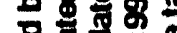

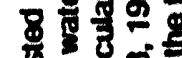
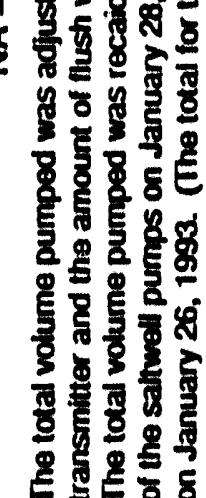

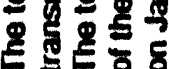


IHHC-EP-0132-62

Thts page intentionally left blank. 
WHC-EP-0182-62

\section{APPENDIX G}

CATCH TANKS AND SPECIAL SURVEILLANCE FACILITIES

$G-1$ 
WHC-EP-0182-62

This page intentionally left blank. 
总

约

3

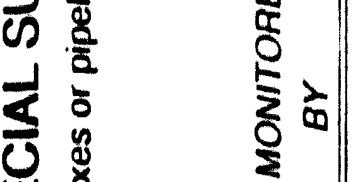

픙

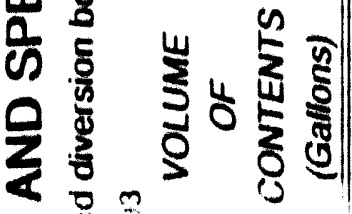

(2)

富

$F$

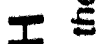

రํㅗㅇ

8

4

II

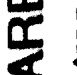

$<$

$\bar{c}$

II

3

른

5

$\omega$

出

京
気
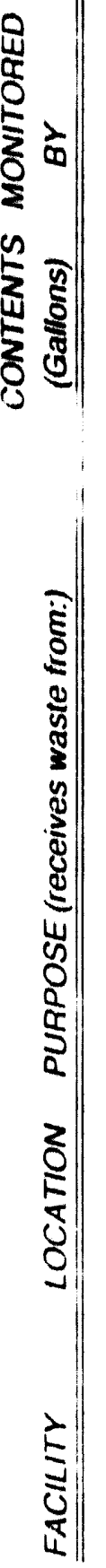

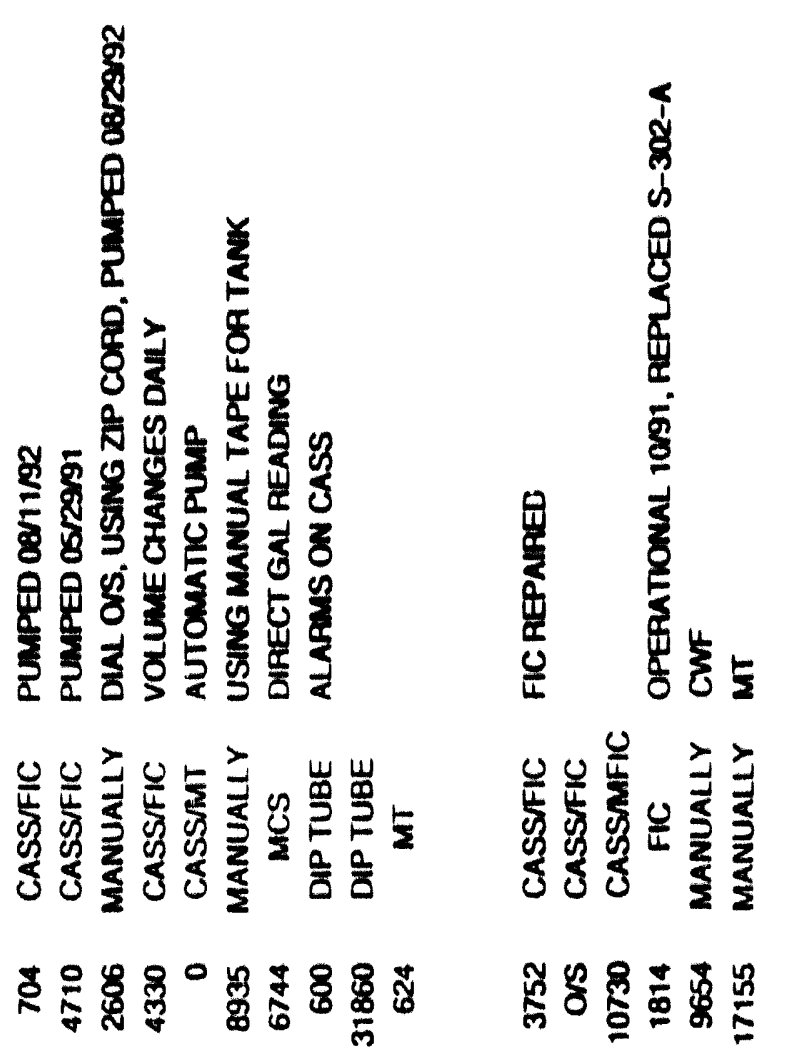

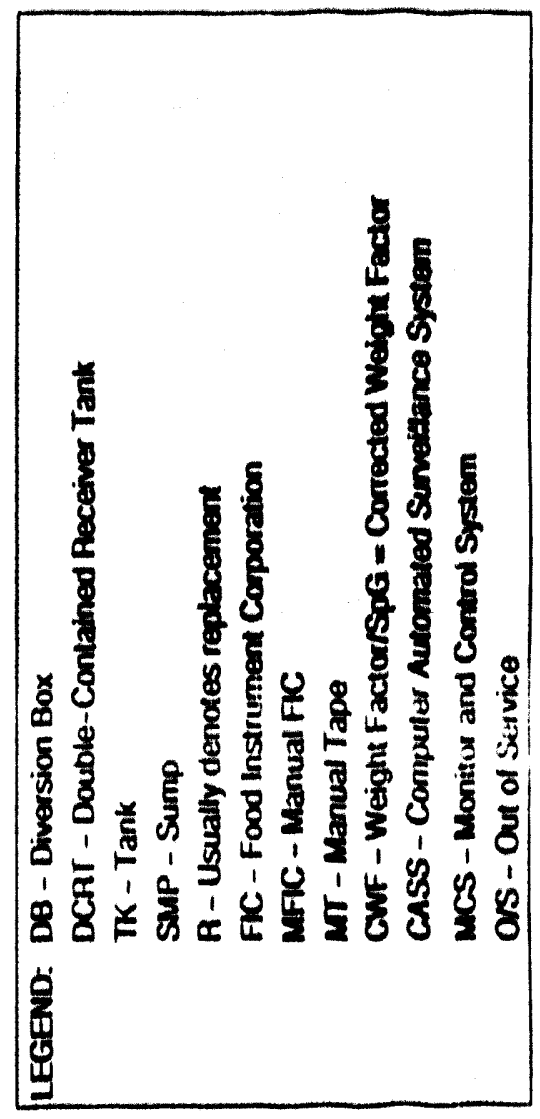

0

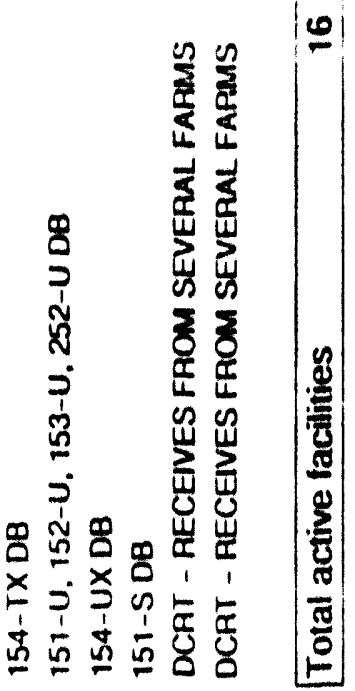

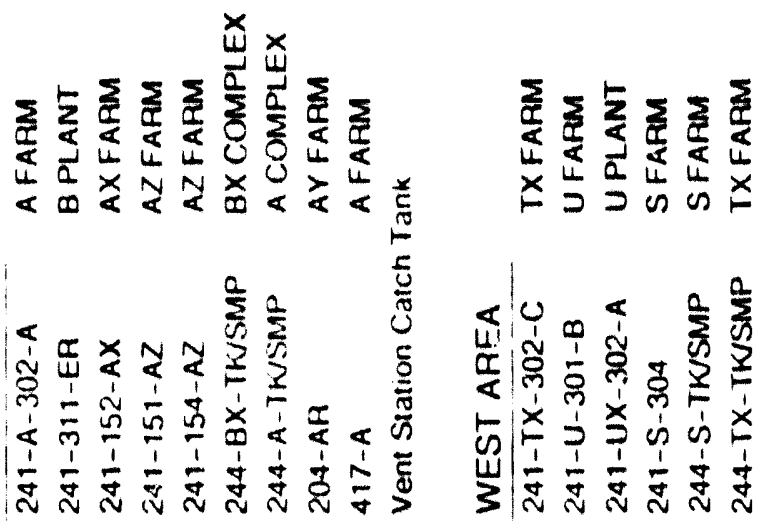

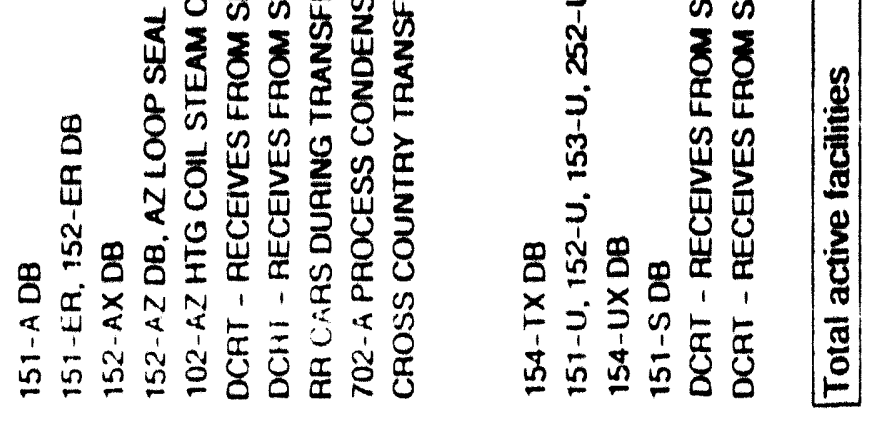




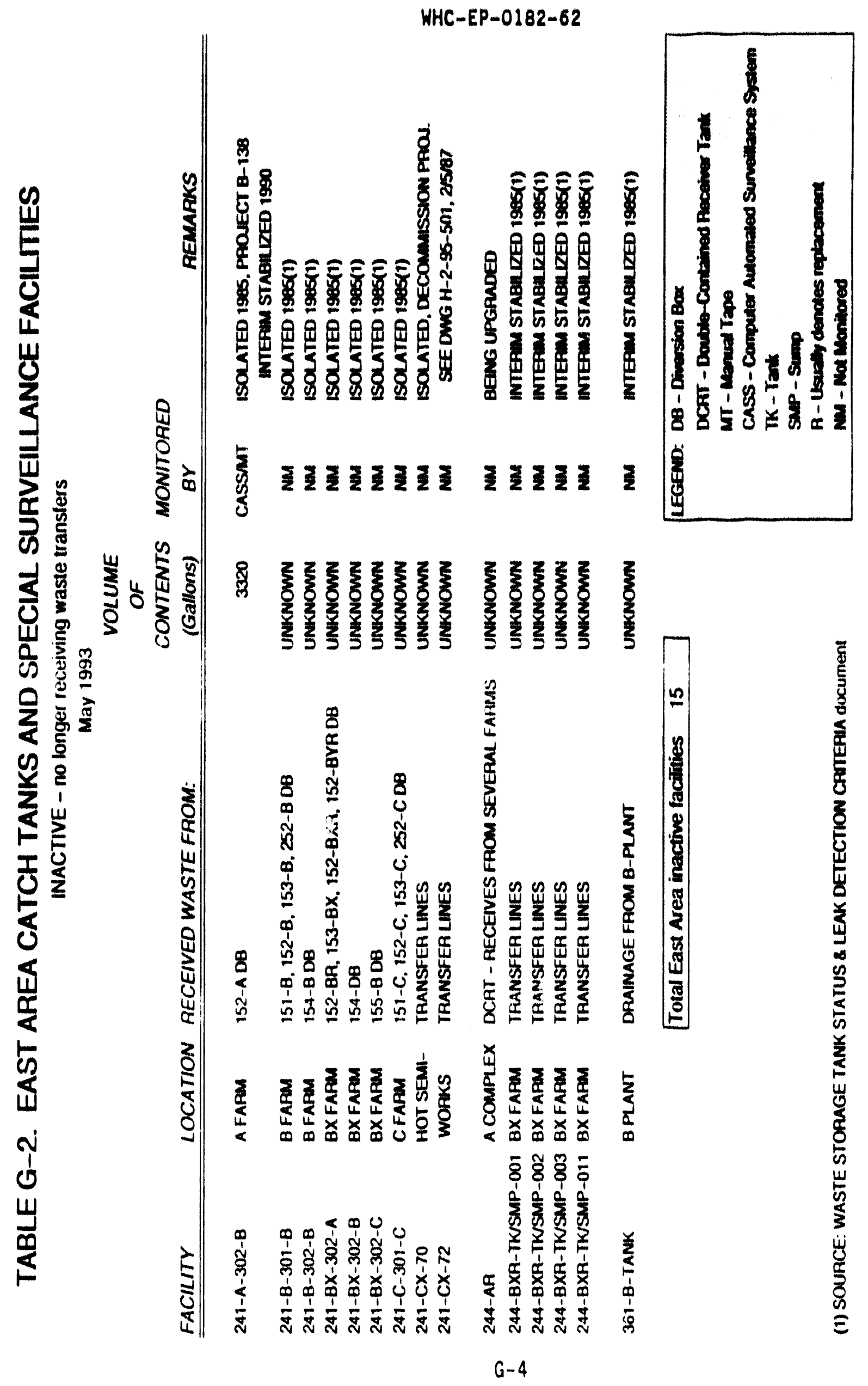


WHC-EP-0182-62
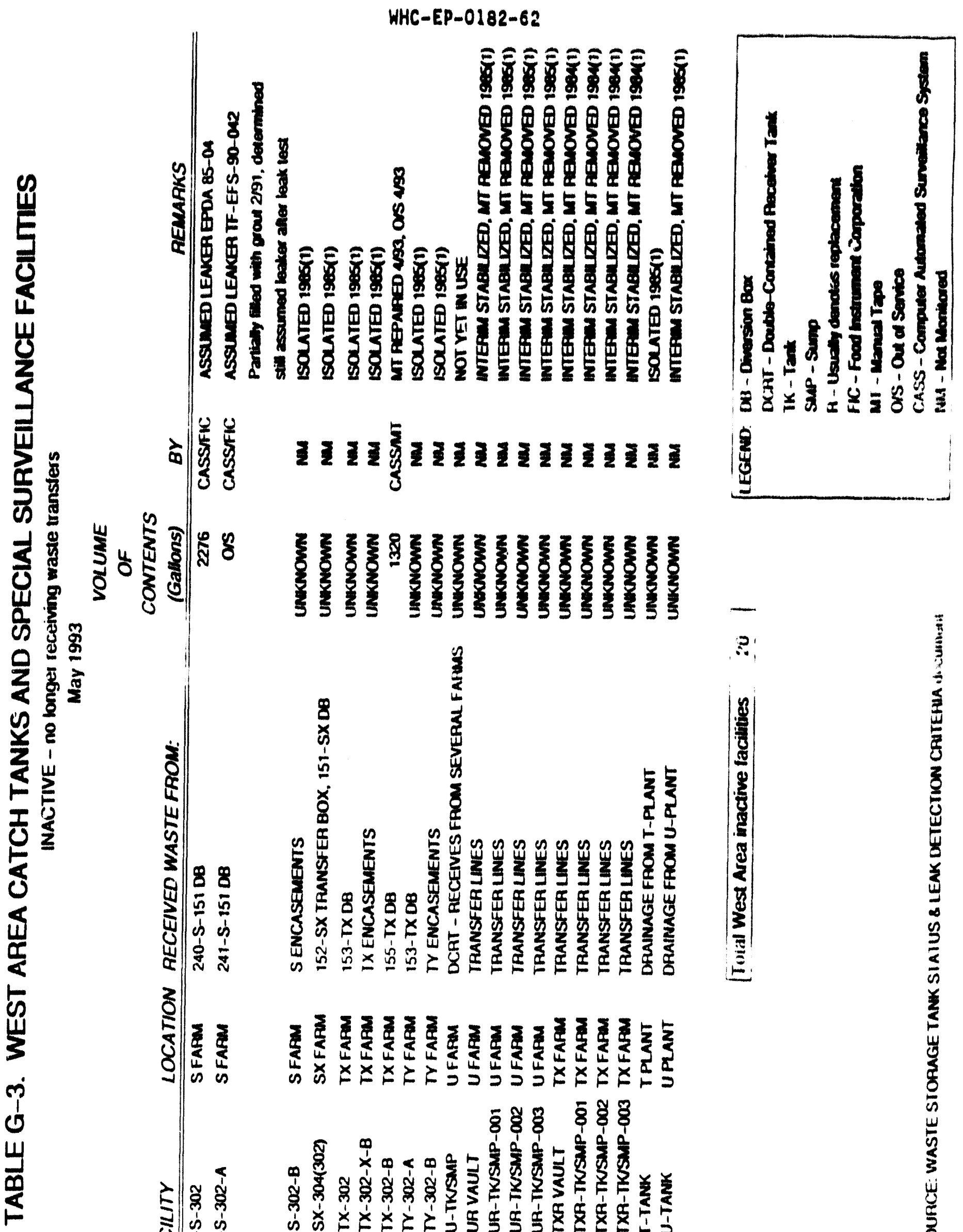
WHC-EP-0182-62

This page intentionally left blank. 
MHC-EP-0182-62

\section{APPENDIX H}

\section{LEAK VOLUME ESTIMATES}

$\mathrm{H}-1$ 
MHC-EP-0182-62

This page intentionally left blank.

$H-2$ 


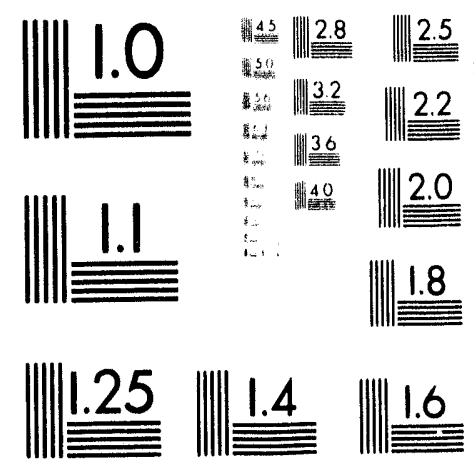



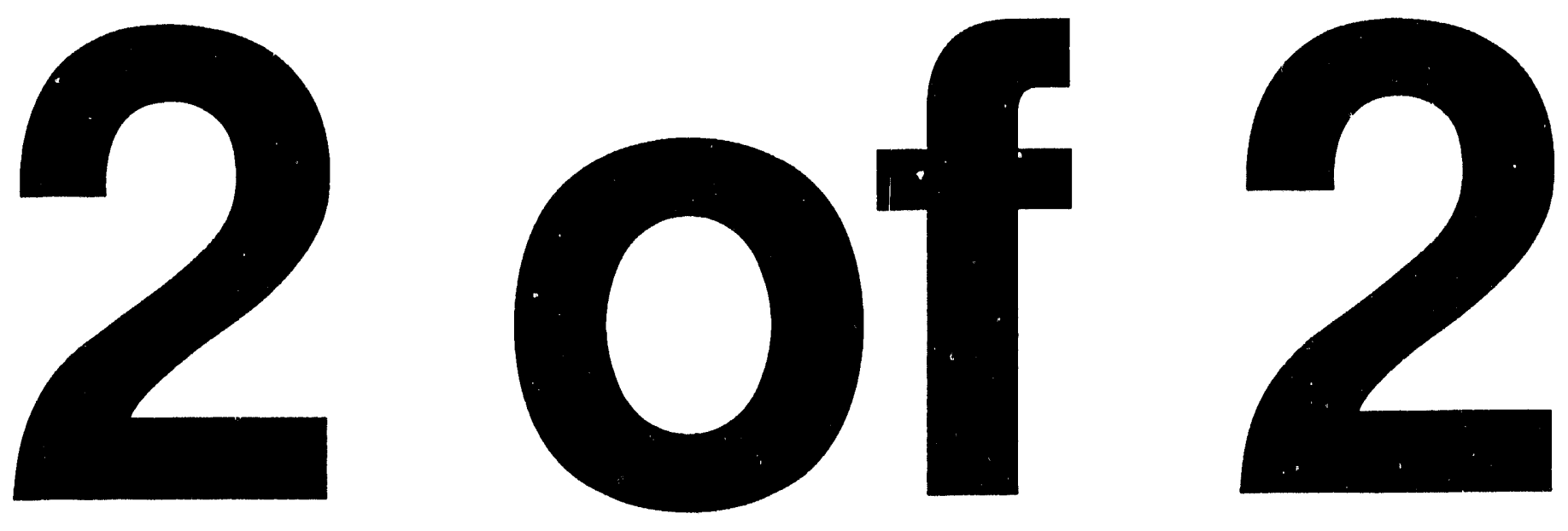
WHC-EP-0182-62

TABLE H-1. SINGLE-SHELL TANK LEAK VOLUME ESTIMATES (4) (Sheet 1 of 5)

Date Declared

Associated

Interim

Confirmed or

Volume (2)(4) KiloCuries

Stabilized

Leak Estimate

Tank No.

Assumed Leaker (3)

(Gallons) $137 \operatorname{cs~(10)~}$

Date

Updated Reference

241-A-103

$241-A-105$

(1)

1987
1975

1975
1963

5500 (9)
500 to 2500
10000 to

277000

$\begin{array}{ll}244-A X-102 & 198 \\ 241-A X-104 & 1977 \\ 241-B-104 & 1974\end{array}$

$241-8-105$

$241-8-107$
$241-8-110$

241-B-111

$241-8-112$

241-B-203

241-B-204

241-BX-101

$241-8 x-102$

$241-8 x-108$

$241-B X-111$

241-BY-103

241-BY-105

241-BY-106

241-BY-107

241-BY-108

241-C-101

241-C-110

$241-C-111$

241-C-202

241-C-203

241-C-204

241-S-104

$241-5 x-102$

$241-5 X-104$

241-SX-107

241-SX-108 (6)

241-SX-109 (6)

241-SX-110

$241-5 X-111$

241-SX-112

241-SX-113

$241-5 X-114$

241-SX-115

241-T-101

241-T-103

241-T-106

241-T- 107

241-T-108

241-T-109

241-T-111

$241-T X-105$

$241-T X-107$

241-TX-110

241-TX-113

$241-T X-114$

$241-T X-115$

$241-T X-116$

241-TX-117

$241-T Y-101$

$241-T Y-103$

241-TY-104

$241-T Y-105$

$241-T Y-106$

$241-U-101$

241-U-104

241-U-110

$241-U-112$

1988

3000

1974

1978

1980

1981

1978

$\begin{array}{ll}1978 & 2000 \\ 1980 & 1200\end{array}$

$\begin{array}{r}1980 \\ 1983 \\ 1984 \\ \hline\end{array}$

1984

197
1974

1971
1974

1976

1984

68 Tanks

$---8$

$\begin{array}{lll}0.8 \text { to } 1.8 & \text { (a) } \\ 85 \text { to } 760 & \text { (b) }\end{array}$

Date

$\begin{array}{lll}8 / 88 & 1987 & \text { (1) } \\ 9 / 78 & 1983 & \text { (a) (a) }\end{array}$

(7)

$\begin{array}{ll}9 / 88 & 1989 \\ 8 / 81 & 1989\end{array}$

$-8$

-

10000 (9)

1973

1984
1984

1972

1980

(5) $\quad 198$

(5) $\quad 1988$

2000

(7)

300 (9)

400 (9)

70000

0000
2500
--8

$--$

50
0.5

1968

1993
1988

1964
(6) $\quad 1962$

(6) $\begin{array}{r}1965 \\ \quad 1976 \\ \hline\end{array}$

1976

1969
1962

1962
1972

500102000

30000

1972

1992
1974
1973

1974

(5)

1974

1977

1977
1984
1977

1974

1974

1977

1977

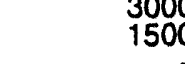

50000

(7)

\begin{tabular}{c}
$3 /$ \\
12 \\
$3 / 2$ \\
12 \\
\hline
\end{tabular}

$3 / 81$
$2 / 85$
$2 / 84$
$3 / 85$
$12 / 84$

195

1989

(b)

1974

1977

$\begin{array}{r}1973 \\ \quad 1981 \\ \hline\end{array}$

1973
1981

1960
1959

1959

1961

1980

$N / A=$ not applicable (not yet Interim stabilized)

FOOTNOTES: SEE NEXT PAGE 
TABLE H-1. Single-Shell Tank Leak Volume Estimates (Sheet 2 of 5 )

Footnotes:

(1) Current estimates (see reference b) are that $610 \mathrm{Kgal}$ of cooling water was added to Tank 241-A-105 from November 1970 to December 1978 to aid in evaporative cooling. In accordance with Dangerous Waste Regulations (Washington Administrative Code 173-303-070 (2)(a)( $i$ i), as amended, Washington State Department of Ecology, 1990, 0lympia, Washington), any of this cooling water that has been added and subsequently leaked from the tank must be classified as a waste and should be included in the total leak volume. In August 1991, the leak volume estimate for this tank was updated and moved into compliance with the WAC regulations. Previous estimates excluded the cooling water leaks from the total leak volume estimates because the waste content (concentration) in the cooling water which leaked should be much less than the original liquid waste in the tank (the sludge is relatively insoluble). The total leak volume estimate in this report $(10 \mathrm{Kgal}$ to $277 \mathrm{Kgal})$ is based on the following (see References).

1. Reference (b) contains an estimate of $5 \mathrm{Kgal}$ to $15 \mathrm{Kgal}$ for the initial leak prior to August 1968.

2. Reference (b) contains an estimate of $5 \mathrm{kgal}$ to $30 \mathrm{Kgal}$ for the leak while the tank was being sluiced from August 1968 to November 1970 .

3. Reference (b) contains an estimate of $610 \mathrm{Kgal}$ of cooling water added to the tank from November 1970 to December 1978 but it was estimated that the leakage was small during this period. This reference contains the statement "Sufficient heat was generated in the tank to evaporate most, and perhaps nearly all, of this water." This results in a low estimate of zero gallons leakage from November 1970 to December 1978.

4. Reference (c) contains an estimate that 378 to $410 \mathrm{Kgal}$ evaporated out of the tank from November 1970 to December 1978. Subtracting the minimum evaporation estimate from the cooling water added estimate provides a range from 0 to $232 \mathrm{Kgal}$ of cooling water leakage from November 1970 to December 1978.

\section{Low Estimate High Estimate}

Prior to August 1968

August 1968 to November 1970

November 1970 to December 1978 Totals

$$
\begin{array}{r}
5,000 \\
5,000 \\
10,000
\end{array}
$$

15,000

30,000

$\frac{232,000}{277,000}$

(2) These leak volume estimates do not include (with some exceptions), such things as: (a) cooling/raw water leaks, (b) intrusions (rain infiltration) and subsequent leaks, (c) leaks inside the tank farm but not through the tank liner (surface leaks, pipeline leaks, leaks at the joint for the overflow or fill lines, etc.), and (d) leaks from catch tanks, diversion boxes, encasements, etc. 
TABLE H-1. Single-Shell Tank Leak Volume Estimates

(Sheet 3 of 5 )

(3) In many cases, a leak was suspected long before it was identified or confirmed. For example, reference (d) shows that Tank 241-U-104 was suspected of leaking in 1956. The leak was "confirmed" in 1961. This report lists the "assumed leaker" date as 1961. Using present standards, Tank 241-U-104 would have been declared as assumed leaker in 1956. In 1984, the criteria designations of "suspected leaker," "questionable integrity," "confirmed leaker," "declared leaker," "borderline," and "dormant," were merged into one category now reported as "assumed leaker." See refertnce (f) for explanation of when, how long, and how fast some of the tanks leaked. It is highly likely that there have been undetected leaks from single-shell tanks because of the nature of their design and instrumentation.

(4) There has been an effort in the past two years to reevaluate these leak volume estimates. During the FY 1993 funding reviews, this reevaluation of leak volumes was given a priority which resulted in this activity no longer being funded. The priority versus funding will be reevaluated as part of the prior to FY 1994 budget planning.

(5) The leak volume estimate date for these tanks is before the "declared leaker" date because the tank was in a "suspected leaker" or "questionable integrity" status; however, a leak volume had been estimated prior to the tank being reclassified.

(6) The increasing radiation levels in drywells and laterals associated with these three tanks could be indicative of a continuing leak or movement of existing radionuclides in the soil. There is no conclusive way to confirm these observations.

(7) Methods were used to estimate the leak volumes from these 19 tanks based on the assumption that their cumulative leakage is approximately the same as for 18 of the 24 tanks identified in footnote (10). For more details see reference $(\mathrm{g})$. The total leak volume estimate for these tanks is $150 \mathrm{Kgal}$ (rounded to the nearest $10 \mathrm{Kgal}$ ), for an average of approximately $8 \mathrm{Kgal}$ for each of the 19 tanks.

(8) The total has been rounded to the nearest $50 \mathrm{Kgal}$. Upperbound values were used in many cases in developing these estimates. It is likely that some of these tanks have not actually leaked.

(9) Leak volume estimate is based solely on observed liquid level decreases in these tanks. This is considered to be the mast accurate method for estimating leak volumes.

(10) The curie content listed is as listed in the reference document and is not decayed to a consistent date; therefore, a cumulative total is inappropriate. 


\section{WHC-EP-0182-62}

TABLE H-1. Single-Shell Tank Leak Volume Estimates. (Sheet 4 of 5 )

\section{References:}

(a) Murthy, K.S., et al, June 1983, Assessment of Single-Shell Tank Residual Liquid Issues at Hanford Site, Washington, PNL-4688, Pacific Northwest Laboratory, Richland, Washington.

(b) WHC, 1991a, Tank 241-A-105 Leak Assessment, WHC-MR-0264, Westinghouse Hanford Company, Richland, Washington.

(c) WHC, 1991b, Tank 241-A-105 Evaporation Estimate 1970 Through 1978, WHC-EP-0410, Westinghouse Hanford Company, Richland, Washington.

(d) Smith, D. A., January 1986, Single-Shell Tank I solation Safety Analysis Report, SD-WM-SAR-006, Rev. 1, Wes'inghouse Hanford Company, Richland, Washington.

(e) McCann, D. C., and T. S. Vail, September 1984, Waste Status Summary, RHO-RE-SR-14, Rockwell Hanford Operations, Richland, Washington.

(f) Catlin, R. J., March 1980, Assessment of the Surveillance Program of the High-Level Waste Storage Tanks at Hanford, Hanford Engineering Development Laboratory, Richland, Washington.

(g) Baumhardt, R. J., May 15, 1989, Letter to R. E. Gerton, U.S. Department of Energy-Richland Operations Office, Single-Shell Tank Leak Volumes, Westinghouse Hanford Company, Richland, Washington.

(h) WHC, 1990a, Occurrence Report "Surface Level Measurement Decrease in Single-Shell Tank 241-AX-102," WHC-U0-89-023-TF-05, Westinghouse Hanford Company, Richland, Washington.

(i) Groth, D. R., Jul j 1, 1987, Internal Memorandum to R. J. Baumhardt, Liquid Level losses in Tanks 241-C-201, -202 and -204, 65950-87-517, Westinghouse Hanford Company, Richland, Washington.

(j) Groth, D. R. and G. C. Owens, May 15, 1987, Internal Memorandum to J. H. Roecker, Tank 103-A Integrity Evaluation, Westinghouse Hanford Company, Richland, Washington.

(k) Campbe11, G. D., July 8, 1988, Internal Memorandum to R. K. Welty, Engineering Investigation: Interstitial Liquid Level Decrease in Tank 241-SX-104, 13331-88-416, Westinghouse Hanford Company, Richland, Washington.

(1) ERDA, 1975, Final Environmental Statement Waste Management Operations, Hanford Reservation, Richland, Washington, ERDA-1538, 2 vols., U.S. Energy Research and Development Administration, Washington, D.C.

(m) WHC, 1992a, Tank 241-SX-108 Leak Assessment, WHC-MR-0300, Westinghouse Hanford Company, Richland, Washington.

(n) WHC, 1992b, Tank 241-SX-109 Leak Assessment, WHC-MR-0301, Westinghouse Hanford Company, Richland, Washington. 


\section{WHC-EP-0182-62}

TABLE H-1. Single-Shell Tank Leak Volume Estimates.

(Sheet 5 of 5 )

(0) WHC, 1992c, Tank 241-SX-115 Leak Assessment, WHC-MR-0302, West inghouse Hanford Company, Richland, Washington.

(p) WHC, 1992d, Occurrence Report, "Apparent Decrease in Liquid Level in Single Shell Underground Storage Tank 241-T-101, Leak Suspected; Investigation Continuing," RL-WHC-TANKFARM-1992-0073, Westinghouse Hanford Company, Richland, Washington.

(q) WHC-1990b, A History of the 200 Area Tank Farms, WHC-MR-0132, Westinghouse Hanford Company, Richland, Washington.

(r) WHC, 1993, Occurrence Report, "Single-Shell Underground Waste Storage Tank 241-BX-111 Surface Leve1 Decrease and Change From Steady State Condition," RL-WHC-TANKFARM-1993-0035, Westinghouse Hanford Company, Richl and, Washington.

(s) WHC, 1993a, Occurrence Report, "Liquid Observation Well (LOW) on Tank 241-SX-102 Indicates Unexpected Interstitial Liquid Level Decrease: Tank Declared an Assumed Leaker," RL-WHC-TANKFARM-1993-0044, Westinghouse Hanford Company, Richland, Washington. 
WHC-EP-0182-62

This page intentionally left blank.

H-8 


\section{DISTRIBUTION}

Number of coptes

OFFSITE - USA

2

Congress of the United States House of Representatives

1111 Longworth Building

Washington, DC 20515-3703

Ron Wyden, Member of Congress, 3rd District Josh Kardon, Legislative Director

U. S. Department of Energy-Headquarters

1000 Independence Avenue, SW

Washington, D. C. 20585

Emile Bernard

H. Calley

H. Eckert

Teresa Fryberger

Sherry Gibson

A. Griffith

L. Gunn

D. Gupta

J. S. Kang

Kenneth Lang

J. C. Lehr

G. Mellinger

C. O'Del1

C. Pepson

J. C. Tseng

S. Woodbury

19901 Germantown Rd, Germantown, MD 20585
R. Lasky
J. Psaras
$\mathrm{EH}-32.1$
NS-20 GTN
P. Worthington

TREV II

TREV II

TREV II

TREV II

TREV II

TREV II $/ 343$

EM-36

EM-36

$E M-351$

EM-36

EM-442

EM-36

$E M-36$

$E M-36$

EM-36

EH-222

TREV II 368

TREV II

TREV II/160

TREV II

TREV II

TREV II

TREV II/366

FORS / 3G-092

U. S. Department of Energy - Oak Ridge Operations Office P. 0. Box 2001 Oak Ridge, TN 37831

W. D. Adams EW-40 
WHC-EP-0182-62

Distribution - continued

U. S. Department of Energy - Savannah River Site

P. 0. Box A

Aiken, SC 29808

C. Anderson

$707-H$

Michael Chandler 703-H

Mazen Shurrab 704-H

T. C. Temple

L. Sjostrom

W. R. West

V. Wheeler

$704-8 \mathrm{H}$

704-S

U. S. Environmental Protection Agency

Region 10

712 Swift Boulevard, Suite 5

Richland, WA 99352

P. T. Day

Washington State Department of Ecology

Nuclear \& Mixed Waste Management Program

P. 0. Box 47600

0lympia. WA 98504-7600

M. T. Gordon

M. Lerchan

Scott McKinney

R. Stanley

Library

7601 W. Clearwater \#102

Kennewick, WA 99336

S. V. Moore

Office Library

Washington State Department of Health

Radiation Protection Section

Industrial Park Building 5, LE-13

Olympia, WA 98504

A. Conkl in

1

General Accounting office

P. 0. Box 321

Richland, WA 99352

C. R. Abraham 


$$
\begin{gathered}
\text { WHC-EP-0182-62 } \\
\text { Distribution - continued }
\end{gathered}
$$

Janet Franco

Oregen State Water Resources Department

Ground Water Hanford Studies

3850 Port land Road

Salem, OR 97310

R. 0. Patt

Lawrence Livermore NationaL Laboratory

Box 808, East Avenue

Livermore, CA 94550

B. C. Hudson

4

Oak Ridge National Laboratory

P. 0. Box 2009

Oak Ridge, TN 37831-6385

C. Forsberg

i. $5-6495$

T. S. Kress

MS- 8088

Bldg 9108

Chemical Technology Division

P. 0. Box 2008

Emory D. Collins

C. Phil McGinnis

6

Los Alamos National Laboratory

P. 0. Box 1663

Los Alamos, NM 87545

\section{Stephen Agnew}

Group INC-14

Phyll is Baca

$\mathrm{J}-514$

T. Larson

C-915

Sylvia Lee

A. Nuels

H. Sullivan

$\mathrm{N}-6$

$\mathrm{N}-6$

Brookhaven National Labol atory Upton, NY 11973

K. K. Bandyopadhyay, Bldg 475-C

M. K. Kaisser, Bldg 475-C

P. D. Kalb, Bldg. 703

M. Reich, Bldg 475-C

J. R. Weeks, Bldg 197-C 


\section{WHC-EP-0182-62}

\section{Distribution - continued}

Brookhaven National Laboratory 1409 Jan Drive

Wilmington, DE 19803

Michael Streicher

Sandia National Laboratories

1515 Eubank, NE

P. 0. Box 5800

Albuquerque, NM 87185

Scott Slezak, Division 6402

Leon D. Chapman, Program Manager

Industrial Waste Reduction Program

1

Massachusetts Institute of Technology

77 Massachusetts Avenue

Cambridge, MA 02139

Mujid S. Kazimi

Professor and Head

Department of Nuclear Engineering

BDM International. Inc. 20030 Century Blvd, Suite 101 Germantown, MD 20874

P. Kiang

K. J. Mahoney

SAIC

Trevion 1, Suite 300 20300 Century B1vd.

Germantown, MD 20874

J. Bunting

J. R. Pearring

R. A. Wullaert

102 Windham Road

Oak Ridge, TN 37830

D. 0. Campbe 11

SAIC

$\frac{\text { } 1845}{184}$ Terminal Drive, Suite 130

Richland. WA 99352

J. Mishima 
WHC-EP-0182-62

Distribution - continued

Harvard School of Public Health

665 Huntington Avenue

Boston, MA 02115

M. First

1

Confederated Tribes, Umatilla Indian Reservation

P. 0. Box 638

Pendleton, OR 97801

Rick George

5

West Valley Nuclear Services Co.

P. 0. Box 191

West Valley, NY 14171

K. K. Gupta

$M S-49$

S. Ketola

$M S-191$

D. K. Ploetz

MS -305

Ram Shukla

Don Stroud

4

Defense Nuclear Facilities Safety Board

625 Indiana Ave. N. N. , Suite 700

Washington, D. C. 20004

Dan Burnfield

Lester Clemons

Paul Gubanc

Suite 700

Dermot M. Winters, Geological Engineer

2

Westinghouse Idaho Nuclear Corporation

P. 0. Box 4000

Idaho Falls, ID 83403-4000

B. Griebenow

MS-5104

A. P. Hoskins

MS -5217

1

C. Abrams

1987 Virginia Drive

Idaho Falls, ID 83404

1

F. Carlson

6965 North, 5 th West

Idaho Falls, ID 8340 l

1

D. Oakley

40912 th Street, SW, $\# 310$

Washington, D. C. 20024 
WHC-EP-0182-62

Distribution - continued

Or. A. Veletsos

Department of Civil Engineering

Rice University

P. O. Box 1892

Houston, TX 77252

1

Westinghouse Electric Corporation

$1801 \mathrm{~K}$ Street NW, 8th floor

Washington DC, 20006

Kevin Billings

Westinghouse Materials Company of Ohio

P. 0. Box 398704

Cincinnati $\mathrm{OH} 45239-8704$

David L. Jacoboski

Senior Engineer, Technology Demonstration

4

Westinghouse Savannah River Company

P. 0. Box 616

Aiken, SC 29802

D. M. Barnes, 773-41A

J. R. Chandler, 703-H

P. d'Entremont

F. G. McNatt, 704-8A

Institute for Energy and Environmental Reseach 6935 Laurel Avenue

Takoma Park, MD 20912

Dr. Arjun Makhijani, President

1

SPAR Aerospace Ltd.

20 Avon Meadow Lane, Suite 220

Avon, CT 06001

Peter W. Kruse

Advanced Technology Systems Division

1

Redzone Robotics, Inc.

2425 Liberty Ave

Pittsburgh, PA 15222-4639

David W. White

12

National Research Counc;il. National Academy of Sciences 2101 Constitution Ave., N. W. Washington D. C. 20418

Robert S. Andrews, Senior Staff officer

Board on Radioactive Waste Management 


\section{WHC-EP-0182-62 \\ Distribution - continued}

1

Converse Consultants

$18 \mathrm{~W}$. Mercer Street, Suite 300

Seattle, WA 98119

David Stanley

1

Brown \& Caldwell

100 W. Harrison

Seattle, WA 98119

Hal Cooper

1

Benton County Department of Emergency Management

P.0. Box 6144

Kennewick, WA 99336

Gary Pira

1

BOVAY Northwest Inc.

660 Swift, Suite $D$

Richland, WA 99352

T. J. McLaughl in

1

Omega Environmental Technology

655 Montgomery Street, Suite 1000

San Francisco, CA 94111

Mike Bailey

1

GEC ALSTHOM Engineering Systems

P. 0 . Box 1274

Richland, WA 99352

J. W. Riddington,

Vice President, Nuclear Marketing

1

T. S. Elleman

North Carolina State University

Department of Nuclear Energy

P. 0. Box 7909

Raleigh, NC 27606

1

Mike Lingle

Stone \& Webster

7677 E. Berry Ave

Englewood, CA 80111

1 Bryant Mather

Corps of Engineers

WESSV-Z

$3909 \mathrm{Ha} l 1 \mathrm{~s}$ Ferry Rd

Vicksburg, MS 39180-6199 


\section{Distribution - continued}

Paul Shewmon

Prof. Metallurgical Engineer

Ohio State University

2477 Lytham Road

Columbus, $\mathrm{OH} 43220$

Waste Management External Advisory Committee Members

Dr. Frank L. Parker

Professor of Environmental and Water Resources Engineering Vanderbilt University

P. 0. Box 1596, Station B

Nashville, TN 37235

Dr. Bruce R. Kowalski

Professor of Chemistry, Co-director of Center for Process

Analytical Chemistry

University of Washington

Chemistry Department, Bidg 10

Seattle, WA 98195

Dr. Greg R. Choppin

Professor of Chemistry

Florida State University

Department of Chemistry, B-164

Tallahassee, FL 32306

Dr. Chester Grelecki

President, Chief Scientist

Hazards Research Corporation

200 Valley Road

Mt. Arlington, NJ 07856

Dr. Alfred Schneider

Professor Meritus of Nuclear Engineering

Georgia Institute of Technology

5005 Hidden Branches Dr.

Dunwoody, GA 30338

Dr. Gary Powers

President

Design Science, Inc.

163 Witherow Road

Sewickley, PA 15143

Ames Laboratory

7 Spedding $\mathrm{Ha} l 1$

Iowa State University

Ames, IA 50011

Bill Haas

R. B. Thompsor 


\section{Distribution - continued}

RKK Ltd.

16404 Smokey Pt. Blvd, Suite 303

Arlington, WA 98223

Chris Reno

1

MACTEC

8320 Centerbrook Place

Alexandria, VA 22308

Stan Blacker

1

Engineering-Science. Inc.

1955 Jadwin Ave, Suite 470

Richl and, WA 99352

Matt Sakach

EBASCO Services, Inc.

1201 Jadwin Avenue, Suite 202

Richland, WA 99352-3429

F. J. Young

1

MTL Systems, Inc.

3481 Dayton-Xenia Road

Dayton, OH 45431-0299

E. McDaniel

Nuclear Consulting Services, Inc. 7000 Huntley Road

P. 0. Box 29151

Columbus, $\mathrm{OH} 43229$

Dr. J. Louis Kovach

1

Battelle Laboratories

505 King Avenue

Columbus, $\mathrm{OH}$ 43201-2693

Rob Taylor Jr., P.E.

Rm 13-6-016

Portland General Electric Co.

121 S. W. Salmon St.

Portland, OR 97204-2991

Wayne Lei, 3WTCBRO5 
WHC-EP-0182-62

Distribution - continued

1

NORCON

7000 Huntley Road

P. 0. Box 29151

Columbus, $\mathrm{OH} 43229$

P. Kovach

$1 \quad$ John Hookins University

1714 Eutaw Place

Baltimore, MD 21217

Morton Corn

1 Roberts Engineering Services, INC.

1980 East Ocean Boulevard

Stuart, FL 34916

Dr. Paul Roberts

1 Columbia River United Technical Consultant

P. 0. Box 912

Bingen, WA 98605

Gregory de Bruler

1 Government Accountability Project

West Coast office

1402 Third Avenue, Suite 1215

Seattle, WA 98101

Thomas E. Carpenter, Director

1 Heart of America Northwest

1305 Fourth Avenue

Cobb Building Suite 208

Seattle, WA 98101

Gerald M. Pollet, Executive Director

\section{OFFSITE - FOREIGN}

1

British Nuclear Fuels Ltd

Risley Warrington

Cheshire WA3 6AS

United Kingdom

Howard A. Edwards

1 Ricardo Hitec Ltd

Club street Works, Bamber Bridge

Preston, PR5 6FN

United Kingdom

P. K. J. Smith 


\section{WHC-EP-0182-62}

\section{Distribution - continued}

1

Telerobot

Consorzto Telerobot

Via Hermada 6

16154 Genova, Italy

Bruno Sessarego

1

SGN

1 , rue des Herons, Montigny-C-Bretonneux

78132 Saint-Quentin-en-Yvelines Cedex,

France

Serge Merlin

2

CEA - Saclay

DCC/DIR

Ba't 121

91190 GIF/Yvette Cedex

France

G. Baudin

R. Atabek

1

Hans Wal ischmiller GmbH

D-7778 Markdorf/Bodel, see

Germany

Wolfgang Wal ischmiller

\section{ONSITE}

U. S. Department of Energy-Richland Operations office
G. E. Bishop
K. W. Bracken
G. J. Bracken
S. T. Burnum
N. R. Croskrey
R. C. Cullison
J. J. Davis
L. Erickson
R. E. Gerton
M. Glasper
W. F. Hendrickson
R. D. Hildebrand
P. E. LaMont
T. Noble
R. L. Person
L. E. Petersen
G. W. Rosenwald
G. H. Sanders
E. J. Senat
A. D. Toth

R3-72

R3-73

R3-72

R3-74

RI -30

A5- 55

R3 -74

R3-74

R3 -72

R3 -72

R3-72

A $5-55$

R3 -74

R3 -72

R3 -72

R3 -72

R3-72

R3 -74

R3 -72

R3-72

Distr-11 
WHC-EP-0182-62

Distribution - continued

W. R. Wrzesinskt

R3-74

J. K. Yerxa

A5-15

Reading Room

A1 -65

Kaiser Engineering Hanford

K. K. Cleveland

S2-10

S. A. Davidson

K6-26

C. J. Denson

E6-51

J. E. Fasso Jr.

S3-10

A. L. Huege 1

E6-31

K. I. Corbin

$\mathrm{R}]-5]$

R. L. Newell

E6-31

D. J. Shrimpton

E6-21

D. T. Vladimiroff

G7-01

Stone \& Webster Engineering Co.

E. L. Richards

R2-83

MACTEC

J. Janus

G6 -18

L. Soler

A4-35

Pacific Northwest Laboratories

D. B. Baird

K $7-34$

D. W. Bennett

K5-22

S. A. Bryan

P7 -25

L. L. Burger

$P 7-25$

J. F. Fletcher

K7 -97

L. K. Holton Jr.

P7 -43

J. Janata

$\mathrm{K} 2-12$

B. M. Johnson

$\mathrm{K} 1-78$

$E$. 0 . Jones

P8-38

A. L. Mitchell

K $7-94$

L. G. Morgan

P7 -35

B. E. Opitz

K6-81

M. S. Peffers

K $7-94$

W. G. Richmond

P7-41

R. D. Scheele

P7-25

E. A. Schmieman

K6- 14

P. A. Scott

P7- 19

J. C. Spanner

D. Strachan

K2-05

$K 2-38$

K. L. Steinmaus

$K 6-84$

R. S. Wegeng

P. D. Whitney

K $7-97$

T. W. Wood

K $7-34$

K6-47 


\section{WHC-EP-0182-62}

\section{Distribution - continued}

\begin{tabular}{|c|c|}
\hline 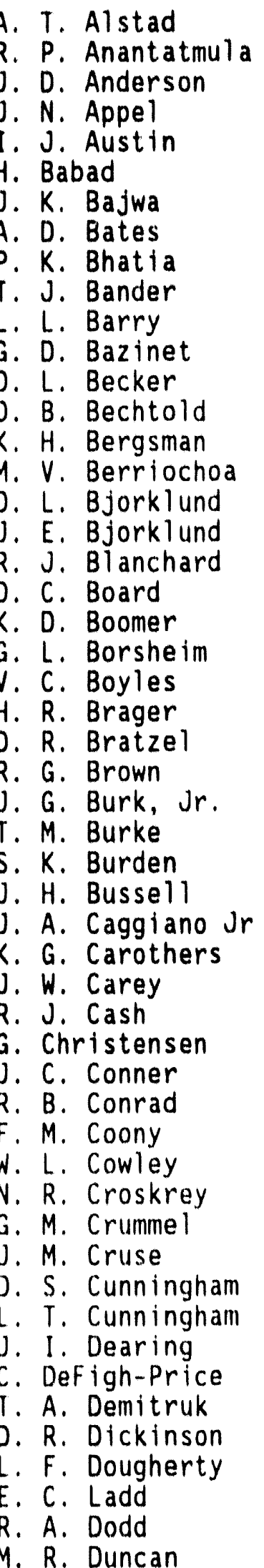 & 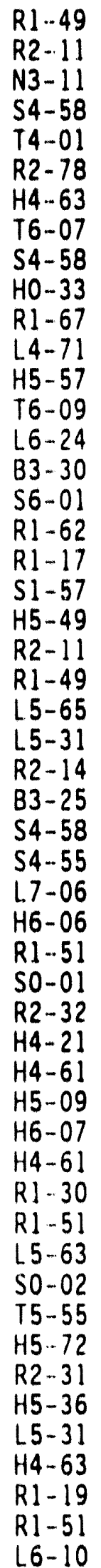 \\
\hline
\end{tabular}




\section{Distribution - continued}

\begin{tabular}{|c|c|}
\hline $\begin{array}{l}\text { L. Dunford } \\
\text { S. Dunnivant } \\
\text { G. Elfner } \\
\text { R. Ellingson } \\
\text { D. Estey } \\
\text { G. Farley } \\
\text { O. Fein } \\
\text { A. Flores } \\
\text { A. Fort } \\
\text { D. Fowler } \\
\text { B. Fowler } \\
\text { L. Fox, Jr. } \\
\text { T. Frater } \\
\text { R. Freeman-Pollard } \\
\text { C. Fulton } \\
\text { A. Gasper } \\
\text { J. Gauck } \\
\text { L. Gilchrist } \\
\text { A. Gilles } \\
\text { D. Godfrey } \\
\text { J. Green } \\
\text { R. Golberg } \\
\text { M. Grigsby } \\
\text { D. Gustavson } \\
\text { W. Hall } \\
\text { S. Haller } \\
\text { G. Hamrick } \\
\text { L. Hampsten } \\
\text { M. Hanlon (30) } \\
\text { M. Hanson } \\
\text { D. Harmon } \\
\text { P. Harris } 111 \\
\text { J. Heard } \\
\text { M. Henderson } \\
\text { W. Hendrickson } \\
\text { G. Hess } \\
\text { C. Higginson } \\
\text { G. Hili } \\
\text { M. Hisaw } \\
\text { J. Holm } \\
\text { D. Hopkins } \\
\text { K. Horsager } \\
\text { D. House } \\
\text { H. Huber } \\
\text { L. Huckaby } \\
\text { L. Humphreys } \\
\text { E. Hysjulien } \\
\text { E. Irwin } \\
\text { N. Islam } \\
\text { D. Johnson } \\
\text { L. Juette } \\
\text { J. Julyk } \\
\text { A. Kirkbride } \\
\text { W. }\end{array}$ & 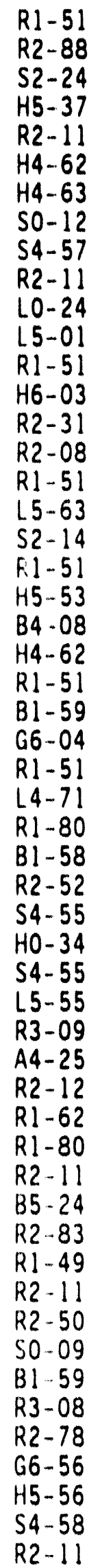 \\
\hline
\end{tabular}




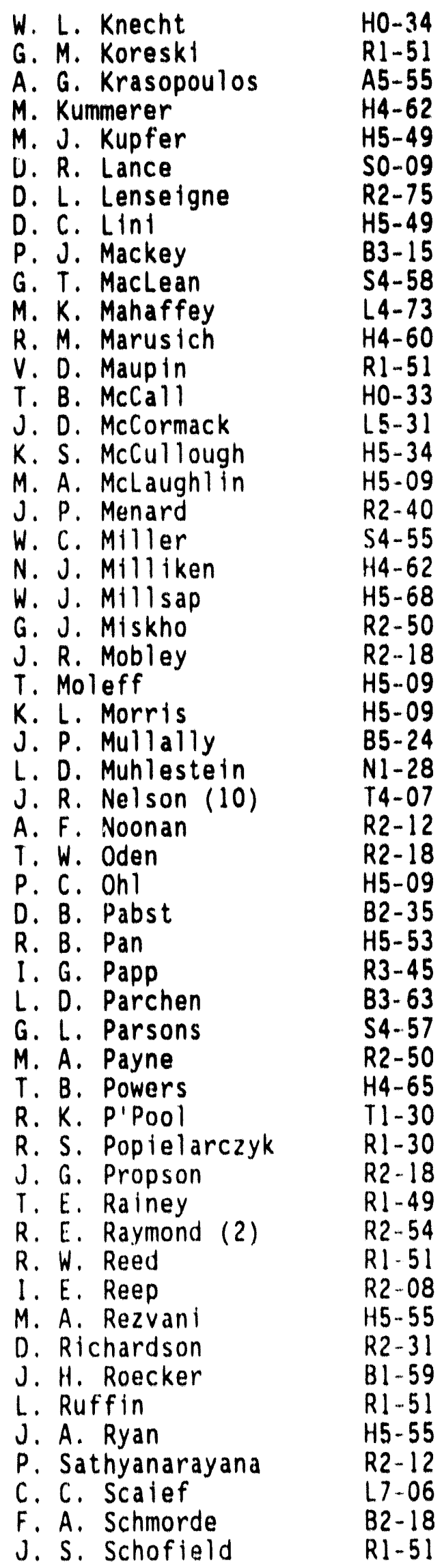




\section{Distribution - continued}

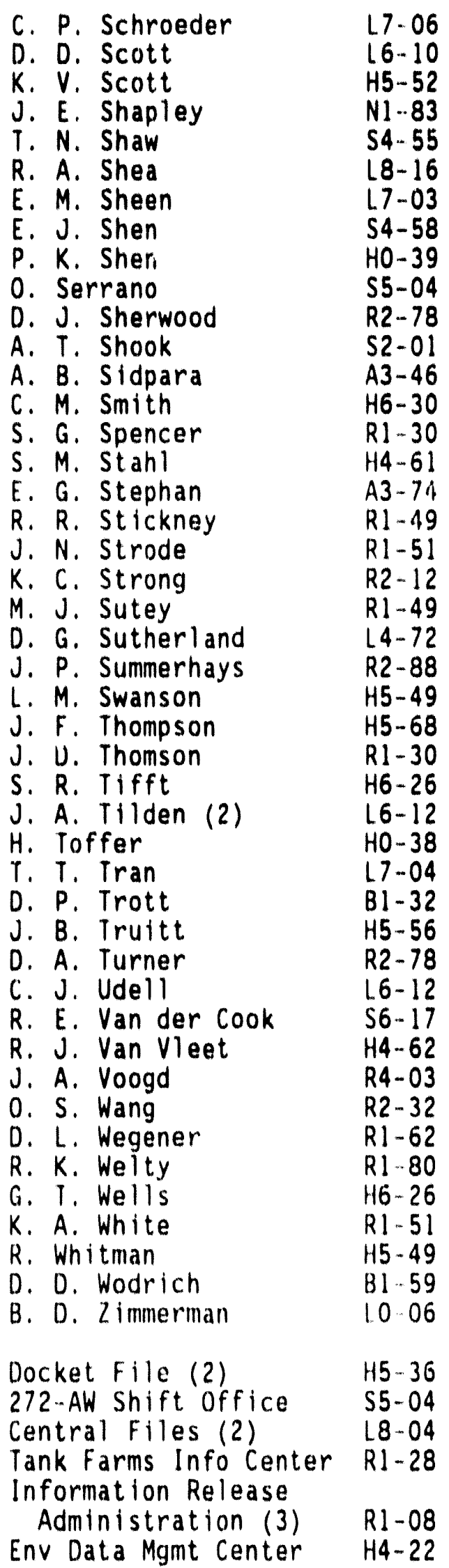



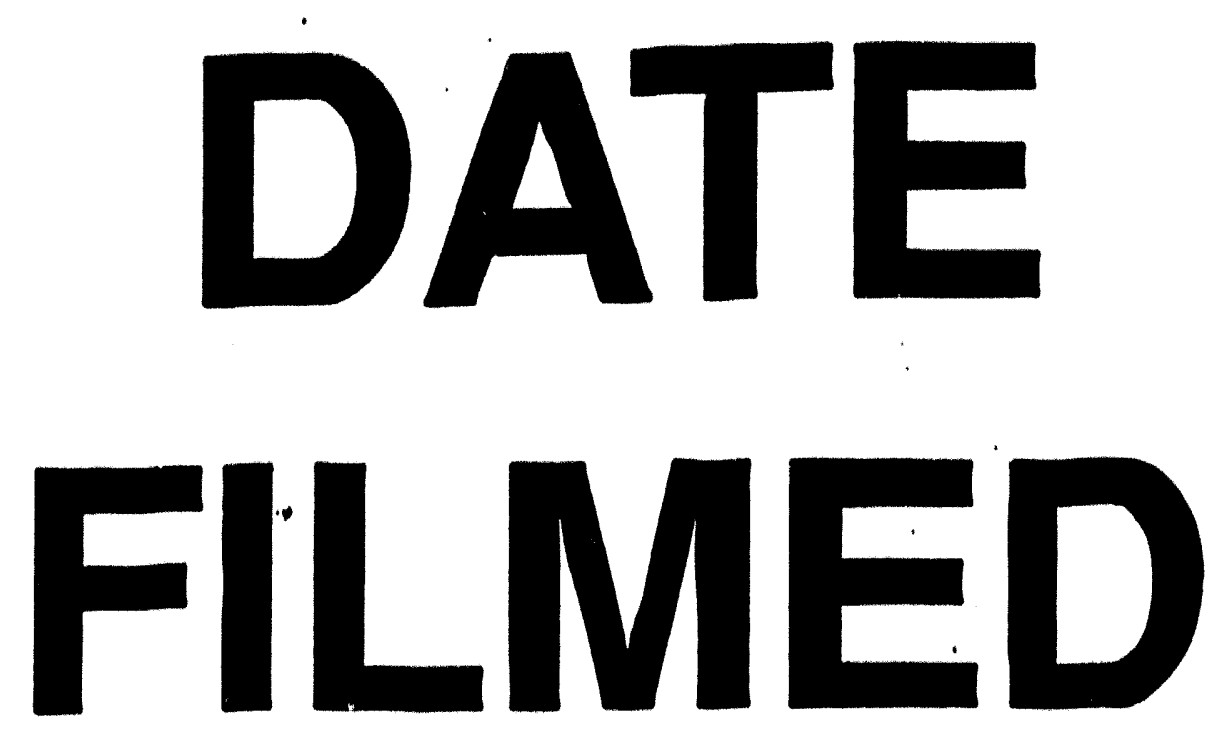

$1 / 21 / 94$
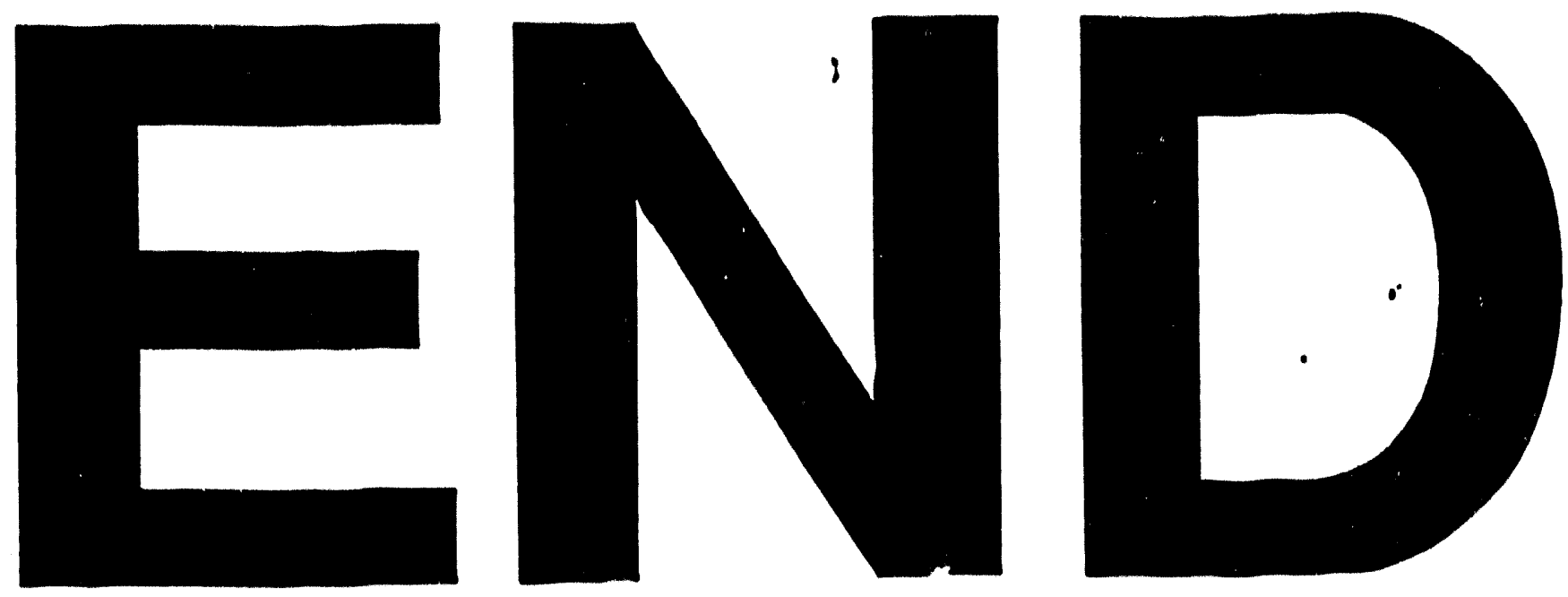
